\title{
Manufacture and \\ Optimization of tubular ceramic membrane supports
}

\author{
Hertzog Bissett \\ B.Sc.(PU vir $\mathrm{CHO}$ ), Hons.Chem.(NWU)
}

November 2005

Dissertation submitted in fulfillment of the

requirements for the degree

Magister Scientiae

at the North-west University

Supervisor:

Assistant Supervisor:
Prof. H. M. Krieg

J. Zah 


\section{Manufacture and optimization of tubular ceramic membrane supports}




\section{ABSTRACT}

Inorganic membranes can be considered an alternative to organic membranes, due to their thermal, chemical and mechanical stability under harsh conditions. Ceramic membranes are used as support structures to increase permeability through composite inorganic membranes in separation processes. Tubular $\alpha$-alumina membrane supports with smooth inner surfaces can be manufactured by means of the centrifugal casting technique.

In this study, the effect of three different AKP powder sizes $(0.25,0.31$ and $0.61 \mu \mathrm{m})$ and sintering temperatures $\left(1000\right.$ to $\left.1400^{\circ} \mathrm{C}\right)$ on the properties of the $\alpha$-alumina supports were investigated in order to determine the optimum particle size and sintering temperature which would yield a porous support with an optimized permeability, while still retaining the smooth inner surface and adequate mechanical strength. A study concerning the possible replacement of the expensive AKP powder range with the less expensive Alcoa CT 3000 SG powder was also undertaken.

The supports manufactured by centrifugal casting were characterized in terms of dimensions and by mercury porosimetry, water permeability and SEM. A novel strength testing apparatus was developed in order to determine the mechanical strength from the inside of the tubular structures. The effect of the polymer concentration, which is added to stabilize the colloidal suspension used in the centrifugal casting technique, as well as the influence of the sintering rate during polymer burn-off, was also investigated.

A larger particle size resulted in an increased porosity, pore size and permeability, while a decrease in linear shrinkage and mechanical strength was observed. There was a decrease in porosity and permeability with increasing sintering temperature while the linear shrinkage and mechanical strength increased. The AKP-30 $(0.31 \mu \mathrm{m})$ and AKP-15 $(0.61 \mu \mathrm{m})$ had a different particle packing than the AKP-50 $(0.25 \mu \mathrm{m})$ supports and consequently a decrease in pore size with increasing sintering temperature was observed for both the AKP-30 and AKP-15 supports, while the pore size remained constant for the AKP-50 supports. Increased polymer concentration 
resulted in an increase in permeability, pore size and porosity, while the mechanical strength of the support decreased. This was due to the evolution of "cracks" during sintering. The sintering rate had no profound influence on the properties of the membrane supports. The powder with the widest particle size distribution (AKP-15 powder) resulted in support structures with the widest pore size distribution. The aim of the study, i.e. the optimization of the supports, was attained when comparing the results in this study to our previous work as well as the available literature. Compared to our own previous study, the permeability increased from 28 to 41 L.h. ${ }^{-1}$ bar. ${ }^{-1} \mathrm{~m}^{-2}$, the porosity from 36 to $37 \%$ and the pore radius from 99 to $167 \mathrm{~nm}$ for the AKP-15 supports sintered at $1200^{\circ} \mathrm{C}$.

Structural cracking and warping during sintering of the powder compacts made from untreated Alcoa CT 3000 SG powder indicated that the particle size distribution (PSD) of the powder was bimodal and hence too wide for centrifugal casted membrane support manufacture. Removal of impurities and powder fractionation by means of both an acid and a column treatment was attempted. The removal of a large amount of fine particles by means of the acid treatment resulted in an improved inner surface of the ceramic supports. Although the size range variation of fractions obtained by the column treatment could not be detected by particle size analysis (Ma/vern Mastersizer), the characterization of three supports, manufactured by combining different fractions, indicated that some degree of fractionation did occur. Characterization of these defect-free supports showed that fractions from the upper section of the column consisted of smaller particles with a narrower PSD compared to fractions from the lower sections of the column. Numerous attempts to repeat these results were, however, unsuccessful, suggesting that the fractionation by means of the acid and column treatments were unpredictable with a low repeatability. Further work is required to obtain a repeatable fractionation, which would be essential in order to prepare centrifugal casted membrane supports using the Alcoa CT 3000 SG powder. 


\section{OPSOMMING}

Anorganiese membrane kan oorweeg word as 'n alternatief vir organiese membrane, as gevolg van hulle termiese, chemiese en meganiese stabiliteit onder ekstreme toestande. Keramiekmembrane word gebruik as ondersteuningstrukture om die permeabiliteit deur saamgestelde anorganiese membrane in skeidingsprosesse te verhoog. Buisvormige $\alpha$-alumina membraanondersteuners met gladde binne-oppervlakke kan vervaardig word deur middel van die sentrifugale deponeringsmetode.

In hierdie studie is die effek van drie verskillende AKP poeiergroottes $(0.25,0.31$ en $0.61 \mu \mathrm{m})$ en sinteringstemperature $\left(1000\right.$ tot $\left.1400^{\circ} \mathrm{C}\right)$ op die eienskappe van die $\alpha$-alumina ondersteuners ondersoek om te bepaal wat die optimum partikelgrootte en sinteringstemperatuur sal wees om ' $n$ poreuse ondersteuner te vervaardig wat geoptimiseerde permeabiliteit sal lewer terwyl die gladde binne-oppervlakte en toereikende meganiese sterkte behou word. 'n Studie aangaande die moontlike vervanging van die duur AKP poeier reeks met die goedkoper Alcoa CT 3000 SG poeier is ook onderneem.

Die ondersteuners wat deur middel van die sentrifugale deponeringsmetode vervaardig is, is gekarakteriseer in terme van sy dimensies en met behulp van kwikporosimetrie, waterpermeabiliteit en SEM. 'n Nuwe sterktetoetsapparaat is ontwikkel om die meganiese sterkte vanuit die binnekant van die buisvormige struktuur te bepaal. Die effek van polimeerkonsentrasie, wat bygevoeg is om die kolloïdale suspensie wat gebruik word in die sentrifugale deponeringsmetode te stabiliseer, sowel as die invloed van die verhittingstempo tydens polimeerafbranding, is ook ondersoek.

'n Groter partikel het 'n verhoogde porositeit, poriegrootte en permeabiliteit tot gevolg gehad, terwyl ' $n$ verminderde liniêre krimping en meganiese sterkte waargeneem is. Daar was ' $n$ verlaging in porositeit en permeabiliteit met toenemende sinteringstemperatuur, terwyl die lineêre krimping en meganiese sterkte toegeneem het. Die AKP-30 $(0.31 \mu \mathrm{m})$ en AKP-15 $(0.61 \mu \mathrm{m})$ het $n$ ander partikelpakking gehad as die AKP-50 $(0.25 \mu \mathrm{m})$ ondersteuners en gevolglik is ' $n$ afname in 
poriegrootte met toenemende sinteringstemperatuur waargeneem vir beide die AKP-30 en AKP-15 ondersteuners, terwyl die poriegrootte konstant gebly het vir die AKP-50 ondersteuners. Toenemende polimeerkonsentrasie het ' $n$ verhoogde permeabiliteit, poriegrootte en porositeit tot gevolg gehad, terwyl die meganiese sterkte van die ondersteuner afgeneem het. Dit was as gevolg van "krake" tydens sintering. Die verhittingstempo het geen waarneembare effek op die eienskappe van die membraanondersteuners gehad nie. Die poeiers met die wydste partikelgrootteverspreiding (AKP-15 poeier) het aanleiding gegee tot die ondersteuner met die wydste poriegrootteverspreiding. Die doel van hierdie studie, d.i. die optimisering van die ondersteuners, is bereik as die resultate in hierdie studie vergelyk word met ons eie vorige werk, asook die beskikbare literatuur. Vergeleke met ons eie vorige werk is daar ' $\mathrm{n}$ toename in permeabiliteit vanaf 28 na 41 L.h. $^{-1}$ bar. $^{-1} \mathrm{~m}^{-2}$, porositeit vanaf 36 na $37 \%$ en porieradius vanaf 99 na $167 \mathrm{~nm}$ vir die AKP-15 ondersteuners wat gesinter was by $1200^{\circ} \mathrm{C}$ waargeneem.

Struktuurkraking en -buiging tydens sintering van die poeierkompak, wat gemaak is van onbehandelde Alcoa CT 3000 SG poeier, het aangedui dat die partikelgrootteverspreiding (PGV) van die poeier bimodaal en as gevolg daarvan te wyd is vir sentrifugaal gedeponeerde membraanondersteunervervaardiging. Verwydering van onsuiwerhede en poeierfraksionering deur middel van ' $n$ suur- en kolombehandeling is onderneem. Die verwydering van die groot hoeveelheid fyn partikels deur middel van die suurbehandeling het 'n verbeterde binne-oppervlak van die keramiekondersteuner tot gevolg gehad. Hoewel die grootteverspreidingsvariasie van die fraksies wat verkry is vanaf die kolombehandeling nie waargeneem kon word deur die partikelgrootte analise nie (Malvern Mastersizer), het die karakterisering van die drie ondersteuners wat vervaardig is van gekombineerde fraksies aangedui dat $n$ mate van fraksionering wel plaasgevind het. Karakterisering van hierdie drie ondersteuners sonder defekte het getoon dat die fraksies uit die boonste gedeeltes van die kolom bestaan het uit kleiner partikels met 'n nouer PGV, vergeleke met die fraksies vanaf die onderste gedeeltes in die kolom. Verskeie pogings om die resultate te herhaal was egter onsuksesvol, wat daarop dui dat die suur- en kolombehandelings tans onvoorspelbaar is, met 'n lae herhaalbaarheid. Verdere werk word benodig om ' $n$ herhaalbare fraksionering te verkry, wat essensieel is om sentrifugaal gedeponeerde membraanondersteuners te vervaardig deur gebruik te maak van die Alcoa CT 3000 SG poeier. 


\section{CONTENTS}

ABSTRACT

$\mathrm{i}$

OPSOMMING

iii

CONTENTS

V

Chapter 1: General Introduction

1

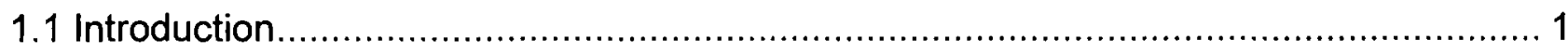

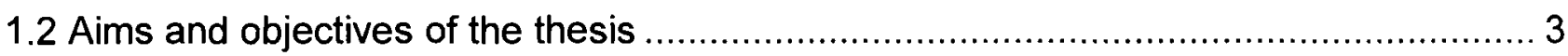

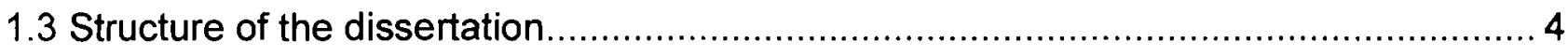

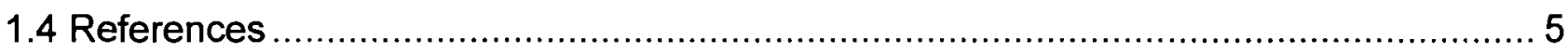

Chapter 2: Ceramic membrane supports ................................................ 7

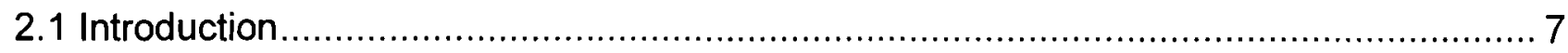

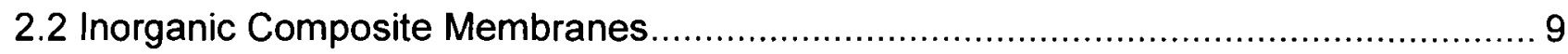

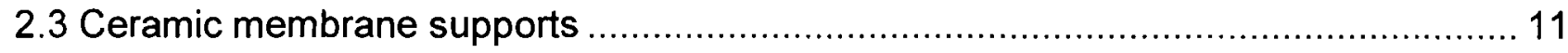

2.4 Centrifugal casted ceramic membrane supports .......................................... 13 


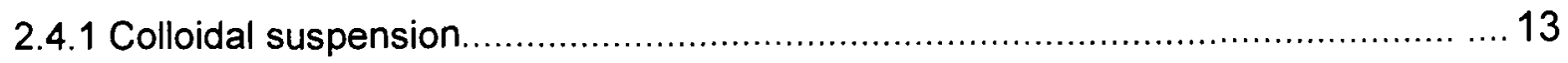

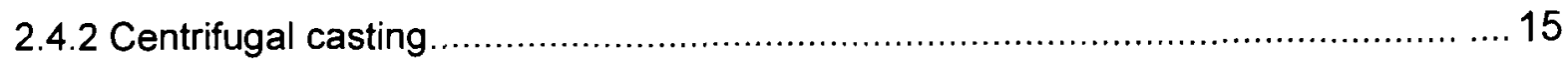

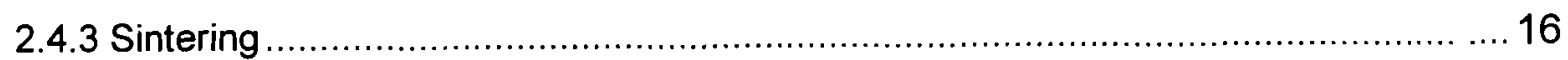

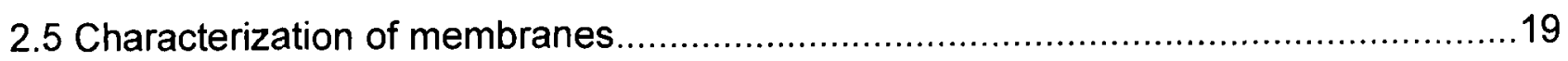

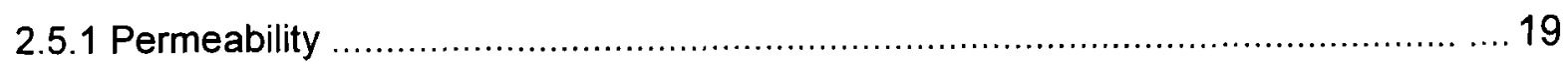

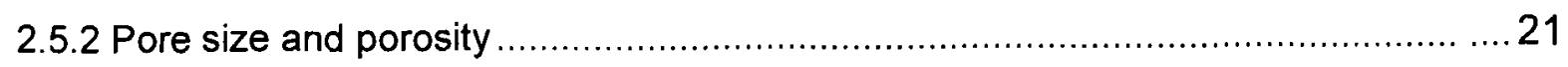

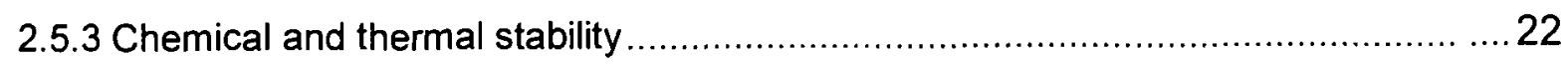

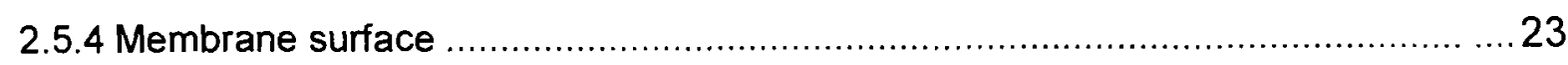

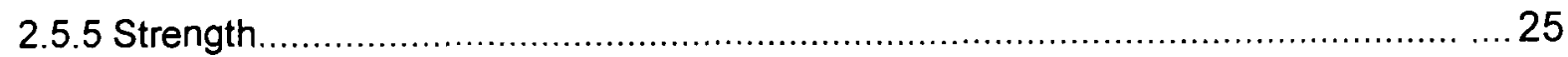

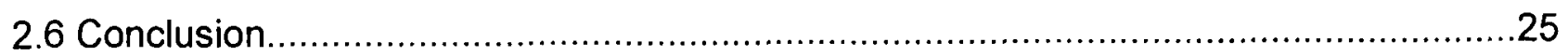

2.7 References

Chapter 3: Optimization of tubular ceramic membrane supports.............34

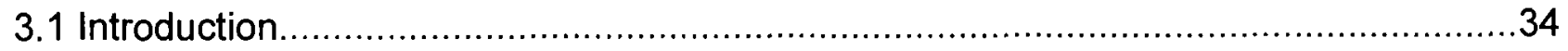

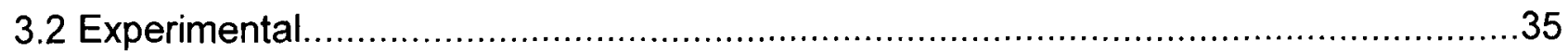

3.2.1 Effect of sintering temperature and powder size ............................................ 35

3.2.2 Influence of dispersant concentration and heating rate......................................37

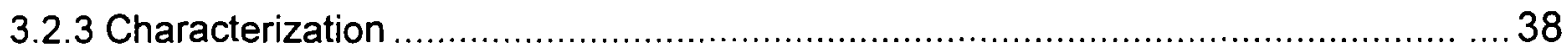

3.2.3.1 Support dimensions and linear shrinkage .......................................................... 38

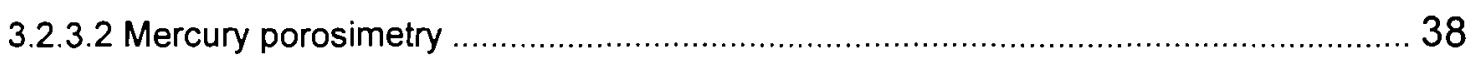

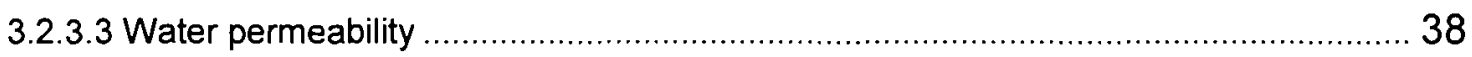

3.2.3.4 SEM

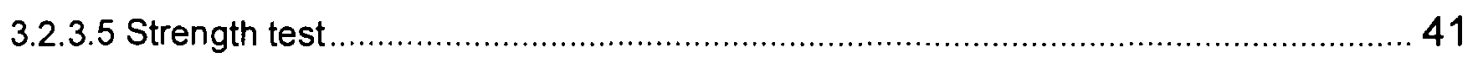

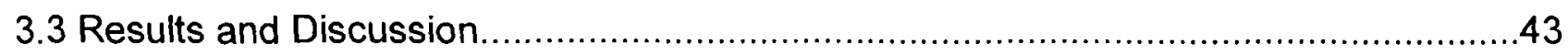

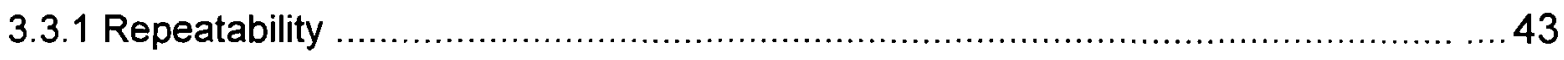


3.3.2 Effect of sintering temperature and powder size ..................................... 43

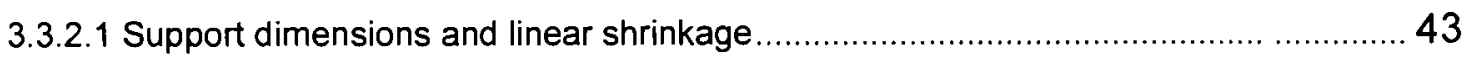

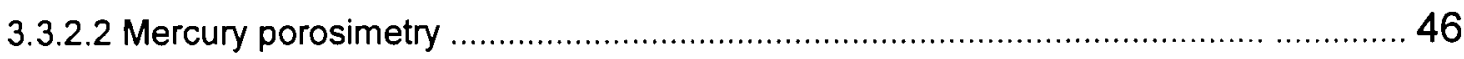

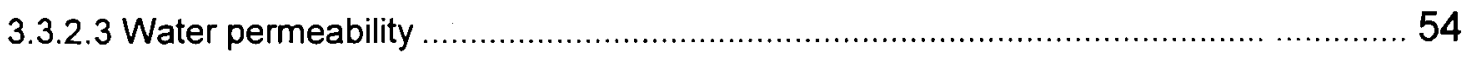

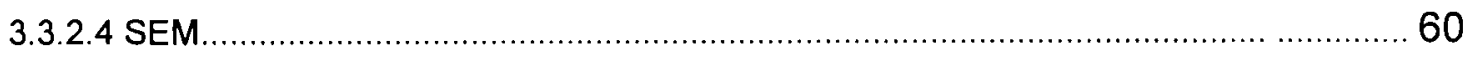

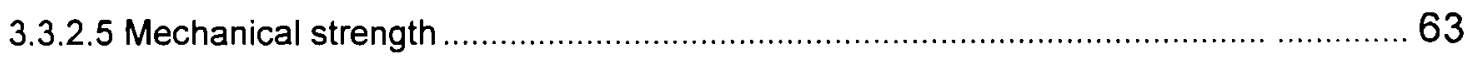

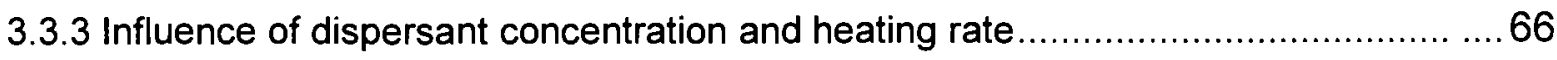

3.3.3.1 Support dimensions and linear shrinkage ......................................6 66

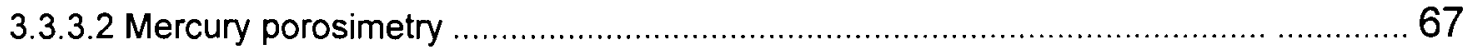

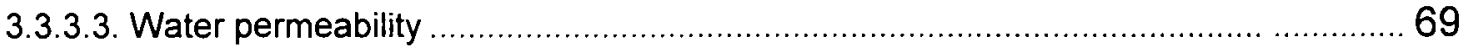

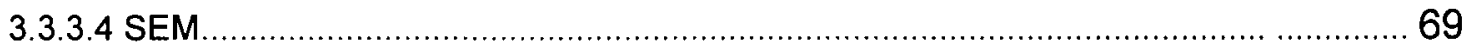

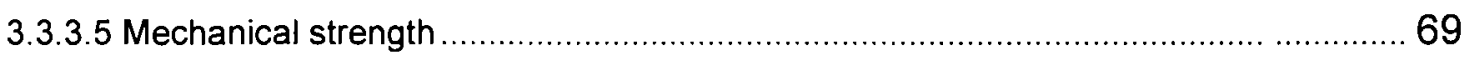

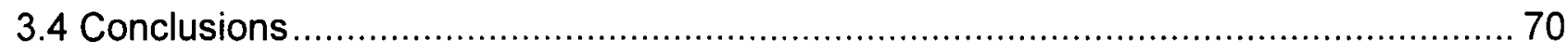

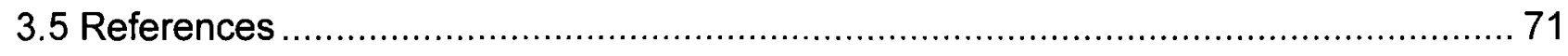

Chapter 4: Manufacture of ceramic supports using Alcoa powder...........75

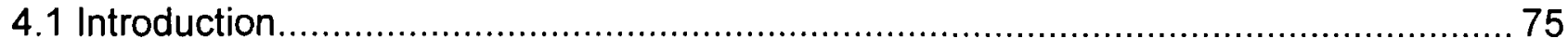

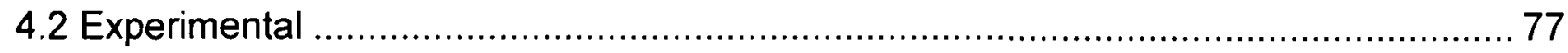

4.2.1 Membrane support manufacture before powder pretreatment .......................... 78

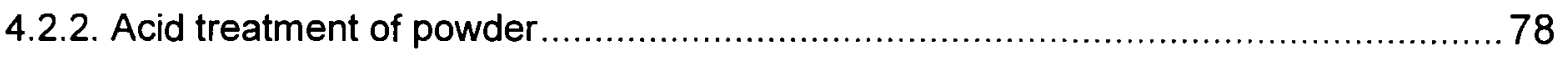

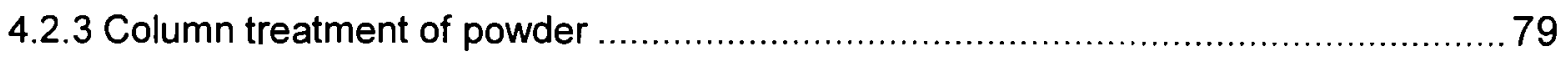

4.2.4 Membrane support manufacture after powder pretreatment ............................. 81

4.2.4.1 Membranes manufactured after acid pretreatment ............................................. 81

4.2.4.2 Membranes manufactured after column pretreatment....................................... 82

4.2.4.3 Membranes manufactured after acid and column pretreatment ............................. 82

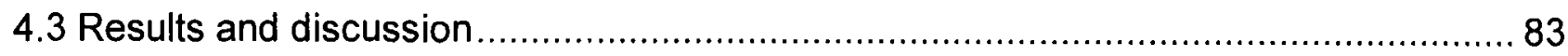


4.3.1 Membrane supports manufactured before powder pretreatment 83

4.3.2 Acid treatment of powder 83

4.3.3 Column treatment of powder 89

4.3.4 Membrane support manufacture after powder pretreatment 92

4.3.4.1 Membranes manufactured after acid pretreatment 93

4.3.4.2 Membranes manufactured after column pretreatment 94

4.3.4.2.1 Mercury porosimetry 94

4.3.4.2.2 Water permeability 96

4.3.4.2.3 Photograph 96

4.3.4.3 Membranes manufactured after acid and column pretreatment. 96

4.3.4.3.1 Support dimensions and linear shrinkage 97

4.3.4.3.2 Mercury porosimetry 98

4.3.4.3.3 Water permeability 100

4.3.3.4 SEM images and photographs 100

4.4 Conclusions 103

4.5 References 105

Chapter 5: Evaluations and Recommendations 106

5.1 General discussion 106

5.2 Conclusion 110

5.3 Recommendations 110

5.4 References 111 


\section{Chapter 1}

\section{General}

\section{Introduction}

\subsection{Introduction}

The application of membrane technology for separation processes in industry is a clean and energy efficient alternative to conventional methods like distillation, physical and chemical adsorption and crystallization. A membrane can be defined as a selective barrier between two phases, where one component of a mixture permeates freely through the membrane, while the permeation of the other component is hindered. ${ }^{1}$ While much emphasis has been placed on polymeric membranes, many industrial separation processes require a membrane with high temperature and chemical stability as well as sufficient strength to endure aggressive environments for which organic membranes are unsuitable. For this purpose, inorganic membranes should be considered. ${ }^{2}$ Since a porous membrane has a high permeance, but low selectivity, while a non-porous membrane has high selectivity, but low permeance, ${ }^{3}$ composite inorganic membranes could be suitable to ensure both high selectivity and permeance 
simultaneously. A composite membrane consists of a porous support membrane which provides the mechanical strength, whilst the thin top-layers (for example zeolites) synthesized onto the support, are responsible for the separation properties of the composite membrane. ${ }^{4}$

The separation of the composite membrane largely depends on the type of top-layer applied onto the support structure. A top-layer that has received extensive attention in recent years is zeolites. Zeolites are microporous and crystalline and are available in a wide variety of well-defined structures for example ZSM-5, NaA, hydroxysodalite and many more. Due to their unique porous properties, zeolites are used in a variety of applications. Major uses are in petrochemical cracking, ion-exchange and in the separation and removal of gases and solvents. They are therefore often referred to as molecular sieves. ${ }^{5}$ In South Africa, an important application for such composite membranes could be in the Fischer-Tropsch process (SASOL) where liquid fuels are made from coal. The Fisher-Tropsch reaction is described by the following equation:

$\mathrm{CO}(g)+2 \mathrm{H}_{2}(g) \longrightarrow-\mathrm{CH}_{2}-(\eta)+\mathrm{H}_{2} \mathrm{O}(g)$

By means of a Zeolite with small pore sizes the various hydrocarbons could be separated from the gas mixture. ${ }^{6}$

Membrane supports made from ceramics are highly suitable for composite membranes due to their inert, strong and temperature resistant properties. The quality of the support surface is crucial to the integrity and thickness of the top-layers. A non-homogeneous top-layer would affect the selectivity while a thick top-layer would result in a decreased permeance. Manufacturing of a ceramic support using centrifugal casting yields an homogeneous, asymmetric support structure with a smooth defect-free inner surface and adequate strength, ${ }^{8}$ resulting in a decreased top-layer thickness and increased permeance whilst still obtaining acceptable selectivity through a composite membrane. The top-layers therefore mainly determine the selectivity of the composite membrane, while the thickness of the top-layers and the properties (pore size and porosity) of the support structure determine the permeance. ${ }^{7,9}$ Optimization of the permeance through the composite membrane could be achieved by increasing the porosity and pore diameter of the support. ${ }^{1}$ Since the suspension properties (including the particle size of the ceramic powder) and the temperature at which the green cast is sintered influence the final support properties, the suspension properties and sintering conditions should be optimized so that a support is obtained that would yield an optimum porosity and pore size whilst maintaining an adequate mechanical strength and a smooth inside surface. ${ }^{1}$

The manufacture of a ceramic support structure by centrifugal casting is as follows: Firstily the ceramic powder is suspended in a liquid and stabilized, after which the suspension is centrifuged 
at high resolutions to compact the ceramic particles into a green cast. The liquid is removed and the green cast dried. The green cast is then sintered at elevated temperatures to obtain a stable structure.

A novel ceramic support with little or no defects could be manufactured using a ceramic powder with a high chemical purity and narrow particle size distribution, for example the AKP $\alpha$-alumina powder range from Sumitomo Chemical Company Ltd. Optimization of the manufacturing procedure would result in a homogeneous membrane with a high porosity and a large median pore diameter. However, the price of the high purity alumina powders with narrow particle size distributions are high and it would therefore be interesting to investigate the possibility of using a less expensive $\alpha$-alumina powder, for example the Alcoa CT 3000 SG powder.

\subsection{Aims and objectives of the dissertation}

The aim of this study was to manufacture tubular ceramic membrane supports with Sumitomo AKP powder by means of the centrifugal casting technique and to optimize the suspension properties and sintering conditions to obtain a membrane which allows maximum permeance whilst retaining a smooth inside surface and adequate mechanical strength. The possibility of replacing the expensive AKP powder with the cheaper Alcoa CT 3000 SG powder was also investigated.

To investigate the influence of the suspension properties and the sintering conditions on the final support structure, a literature study was undertaken to asses the various methods employed to improve the water permeance rate though the ceramic supports. After the literature study it was decided to investigate the influence of 3 AKP powder sizes $(0.25,0.31$ and $0.61 \mu \mathrm{m})$ and the influence of the sintering temperature on the support structure. A temperature range from $1000^{\circ} \mathrm{C}$ to $1400^{\circ} \mathrm{C}$ was used. The various supports were characterized in terms of dimensions, mercury porosimetry, water permeability and SEM. To evaluate the strength of the supports, a novel strength testing apparatus was developed which determines the mechanical strength from the inside of the tubular structures. The concentration of the polymer (APMA) which was used to disperse the $\alpha$-alumina powder in water, as well as the sintering rate used to burn off the polymer was also investigated. Two polymer concentrations $(0.204 \mathrm{~g} / \mathrm{ml}$ and $0.102 \mathrm{~g} / \mathrm{ml})$ and two sintering rates $\left(0.4^{\circ} \mathrm{C} / \mathrm{min}\right.$ and $\left.1.0^{\circ} \mathrm{C} / \mathrm{min}\right)$ were used. 
A similar approach was used when manufacturing a support from the cheaper Alcoa powder, except that the powder had to be pretreated in order to make it suitable for tubular support production. It was found that the particle size distribution of the Alcoa CT 3000 SG powder was bimodal, which led to severe cracking and warping of the support structure during sintering. The powder was firstly pretreated in an acid medium to remove many of the fine particles present. Secondly, the powder was fractionated by means of gravitational settling in a fractionating column. Various conditions were investigated both for the acid and column treatment in order to obtain maximum fractionation of the powder. The fractions were characterized by means of Particle Size Analysis and SEM. Several of the fractions were used to prepare supports and successfully produced supports were characterized according to dimensions, mercury porosimetry, water permeability and SEM. Defect-free and faulty supports were also photographed.

\subsection{Structure of the dissertation}

The advantages of inorganic membranes compared to organic membranes and the methods and theory of obtaining a tubular membrane that allows maximum permeance with acceptable selectivity are discussed in Chapter 2. This includes a discussion on the suspension used in the manufacturing procedure, the fundamental aspects of the centrifugal casting technique, the mechanisms occurring during sintering of the green cast and lastly the methods employed to characterize the supports.

In Chapter 3 the optimization of the AKP powder range is presented. This includes the study on the influence of the particle size and sintering temperature. Finally, the influence of the polymer concentration and sintering rate on the properties of the supports is presented.

A feasibility study on the possibility of replacing the expensive AKP powder range with the Alcoa powder is presented in Chapter 4.

Finally, an evaluation and summary of the thesis is given in Chapter 5. 


\subsection{References}

'M.Mulder, Basic principles of membrane technology, second edition, Kluwer Academic Publishers, 1996, Chapter 1: Introduction, pp. 7

${ }^{2}$ J.M.Benito, A.Conesa, F.Rubio, M.A.Rodriguez, Preparation and characterization of tubular ceramic membranes for treatment of oil emulsions, Journal of European Ceramic Society, 25, 2004, 1895

${ }^{3}$ C.Bai, M.Jia, J.L.Falconer, R.D.Noble, Preparation and separation properties of silicalite composite membranes, Journal of Membrane Science, 105, 1995, 79

${ }^{4}$ R.M. de Vos, H.Verweij, High-selectivity, high-flux silica membranes for gas separation, Journal of Membrane Science, 279, 1998, 1710

5 J.García-Martinez, D.Cazorla-Amorós, Á.Linares-Solano, A.B.Fuertes, Silicalite-1 membranes supported on porous carbon discs, Microporous and Mesoporous Materials, 59, 2003, 147

${ }^{6}$ http://chemed.chem.purdue.edu/genchem/topicreview/bp/1organic/coal.html

${ }^{7}$ A.Jena, K.Gupta, Porosity characterization of microporous small ceramic components, Porous Materials Inc., 2001

${ }^{8}$ A.Nijmeijer, C.Huiskes, N.G.M.Sibelt, H.Kruidhof and H.Verweij, Centrifugal casting of tubular membrane supports by centrifugal casting, American Ceramic Society Bulletin, 77, 1998, 95

${ }^{9}$ P.M.Biesheuvel, V.Breedveld, A.P.Higler and H.Verweij, Graded membrane supports produced by centrifugal casting of a slightly polydisperse suspension, Chemical Engineering Science, 56(11), 2001, 3517 


\section{Chapter 2}

\section{Ceramic membrane}

\section{supports}

\subsection{Introduction}

Separation processes are some of the most important operations in the chemical industry for obtaining pure chemical substances from mixtures or reactions systems. A vast range of separation technologies are in use in industry today and include distillation, physical and chemical adsorptions and crystallization processes. Most of these separation technologies are expensive and energy consumption is high. As energy costs rise, membrane technology is likely to play an increasingly important role in reducing the environmental impact and costs of industrial separation processes. ${ }^{1}$ 
Separation by means of membranes is a relatively new development and was not considered technically important until about 33 years ago. ${ }^{1}$ Membranes can be used for concentration, purification, fractionation and reaction mediation. About $20 \%$ of membranes employed in industry are used in reactions, while $80 \%$ are used for separation. The most common separation processes in use today are ultrafiltration, nanofiltration, gas separation, pervaporation and electrodialysis. The application of membrane processes have recently appeared in new fields of industry such as new energy sources, petrochemistry and special applications which concern protecting the environment. Membranes with specific combinations of features (resistance to abrasion and corrosion) are required for these purposes. ${ }^{1}$

A membrane can be defined as a selective barrier between two phases. In separation applications the goal is to allow one component of a mixture to permeate through the membrane freely, while hindering permeation of other components, which can be attained due to differences in physical and/or chemical properties of the components.

Figure 2.1 illustrates the membrane process principle. The feed contains a number of components, which is fed into the membrane module. Depending on the characteristics of the membrane, certain components would not permeate through the membrane, or permeate at a slower rate than other components. These components would remain in the retentate, while the component which permeates freely though the membrane or at a high rate would then be present in the permeate.

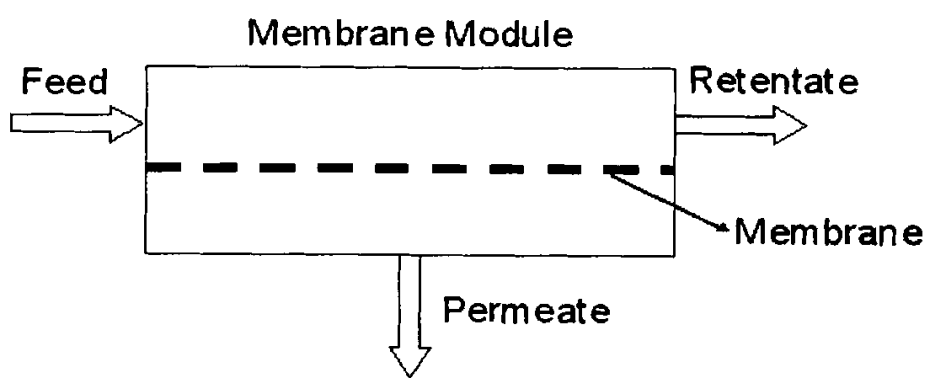

Figure 2.1 Membrane module.

The most important characteristics of a membrane are:

- Selectivity

- Permeability

- Stability 
Two parameters are used to express the selectivity of a membrane namely:

- Rejection

- Separation factor

From Figure 2.1 the rejection factor $(R)$ can be calculated as follows:

$$
R=\frac{C_{f}-C_{p}}{C_{f}}=1-\frac{C_{p}}{C_{f}}
$$

where $C_{f}$ is the feed concentration and $C_{p}$ is the permeate concentration. The separation factor (a) on the other hand is expressed as:

$$
\alpha=\left(\frac{\frac{y_{a}}{y_{b}}}{\frac{x_{a}}{x_{b}}}\right)
$$

where $y_{a}$ and $y_{b}$ are the concentrations of components $A$ and $B$ in the permeate, and $x_{a}$ and $x_{b}$ the concentrations of components $A$ and $B$ in the feed.

Two types of synthetic membranes are available for separation applications, namely polymeric and inorganic membranes. While polymeric membranes have low thermal, chemical, and mechanical stability, ${ }^{2}$ inorganic membranes possess superior structural stability, for example in terms of swelling or compaction. ${ }^{3}$

Table 2.1 lists a few of the advantages and disadvantages of inorganic membranes. In spite of the numerous disadvantages, ${ }^{2}$ the volume of research on inorganic membranes has increased considerably due to their application capabilities in harsh environments where organic membranes suffer changes in their structure. ${ }^{10}$ 
Table 2.1 Advantages and disadvantages of inorganic membranes compared to polymeric membranes

\begin{tabular}{cc}
\hline \multicolumn{1}{c}{ Advantages } & Disadvantages \\
\hline High thermal stability at high temperatures & High production cost \\
Resistance to harsh environments & Brittleness \\
Resistance to high pressure drops & Low membrane surface per volume module \\
Inertness to microbiological degradation & $\begin{array}{c}\text { Difficulty to achieve high selectivities on a } \\
\text { large scale }\end{array}$ \\
Easy to clean after fouling & $\begin{array}{c}\text { Low permeability of high-selectivity (dense) } \\
\text { membranes at medium temperatures } \\
\text { Difficulty sealing membrane-to-module at } \\
\text { high temperatures }\end{array}$ \\
Long life-time & Low crack resistance \\
\hline
\end{tabular}

\subsection{Inorganic Composite Membranes}

Inorganic membranes are usually multi-layered systems, which means that they consist of a support and one or more top-layers as illustrated in Figure 2.2. The support provides the mechanical strength, whilst the top-layers are responsible for the separation properties of the membrane. The top-layers can consist of various types of inorganic structures such as for instance, zeolites, depending on the purpose of the membrane. The top-layers and the support have to be stable at elevated temperatures and resistant to solvents in order to be used effectively for an extensive period of time. ${ }^{4,5}$ 


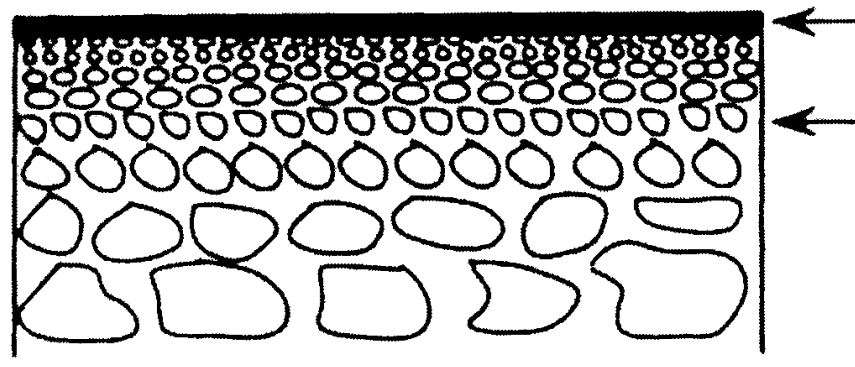

Selective layer

\section{Support}

Figure 2.2 Composite membrane.

Two essential properties of membranes are the flux through the membrane and the selectivity (separation factor). In order to maximize the flux through the composite membrane with acceptable separation, the flux through the support has to be optimized and the thickness of the top-layer (for example a zeolite) has to be minimized. According to Jena et al., the performance of a ceramic support is mainly determined by the pore size and pore size distribution ${ }^{6}$. The optimization of the composite membrane (support and top-layer) is therefore achieved by increasing the porosity of the support whilst decreasing the thickness of the top-layers. ${ }^{7}$

Although many materials can be used to produce inorganic membrane supports, ceramics, ceramic composites and stainless steel have high strength characteristics. As strength is critical for operating under extreme mechanical and thermal conditions, the use of ceramics and stainless steel in inorganic membrane production is a major advantage. ${ }^{8}$

Supports produced from ceramics have, however, a few advantages compared to stainless steel supports. The surface of stainless steel supports is generally rougher, requiring a thicker separation layer, resulting in a decreased permeation through the composite membrane. Another disadvantage is that stainless steel supports have a higher thermal expansion coefficient. The expansion coefficient difference between for example zeolite and ceramic is less than the difference between zeolite and stainless steel and the stainless-steel-supported zeolite therefore tends to be more prone to thermal cracking and adhesion problems. Both the above mentioned problems make it harder to produce a thin defect free separation layer directly onto a porous stainless steel support. ${ }^{9}$ 


\subsection{Ceramic membrane supports}

Authors have used various methods to improve the porosity or change the pore size distribution and pore size of ceramic structures. ${ }^{10,11,32}$ One of these methods is to prepare an alumina suspension containing carbon particles of desired diameters. ${ }^{10}$ As illustrated in Figure 2.3 , the carbon particles $(C)$ are orientated in between the alumina particles in the compact. During sintering, the carbon particles are removed and pores of various sizes are produced. By changing the size and/or amount of carbon in the starting powder the porosity, pore size and pore size distribution of the sintered ceramic structure can be altered. ${ }^{10}$

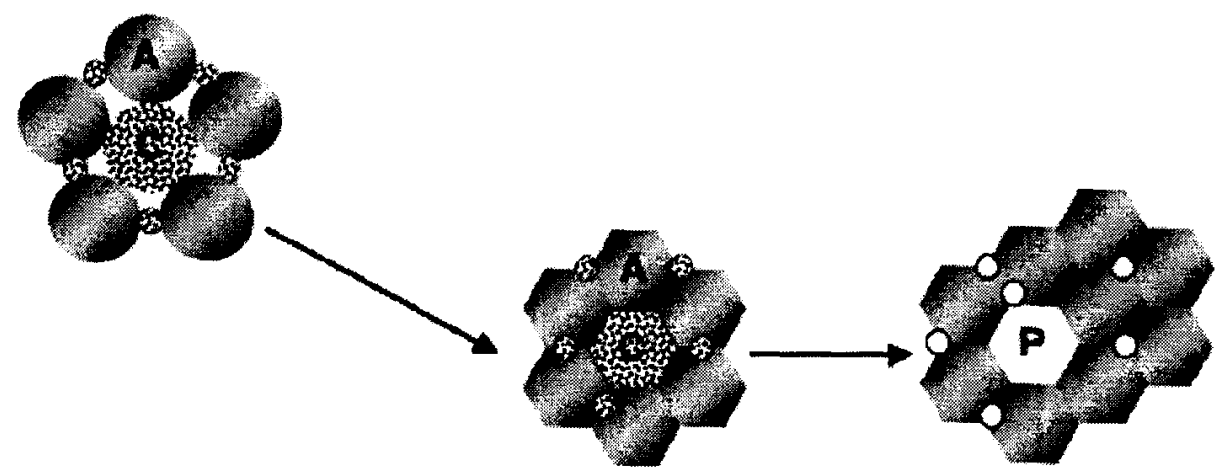

Figure 2.3 Addition of carbon to starting suspension in order to alter the characteristics of a ceramic structure.

The shape of the pores can also be changed. In the previous example, spherical carbon particles were added and rounded pores were obtained. Isobe et al. ${ }^{11}$ made use of carbon fibers to produce unidirectional-orientated pores by extrusion as illustrated in Figure 2.4.

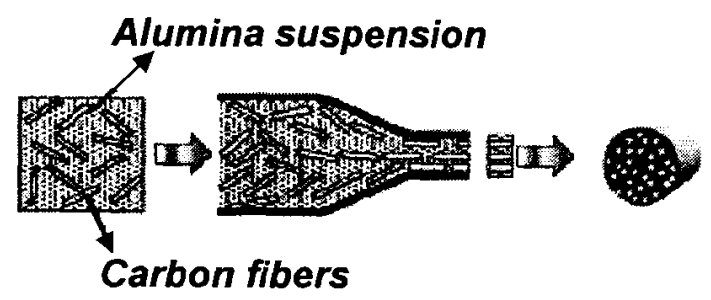

Figure 2.4 Unidirectional-orientated pores produced by extrusion. 
Depending on the purpose of the membrane, various methods are used to apply the top-layers onto the support. For example, for gas separation an amorphous silica top layer of $\sim 30 \mathrm{~nm}$ can be obtained using the in-situ hydrothermal synthesis method. While the silica has very small pore sizes, suspension coatings with $\gamma$-alumina typically produce pore sizes of 0.2 to $1 \mu \mathrm{m}$, making the membrane suitable for microfiltration. ${ }^{12,13}$

There are various methods available for producing supports, for example extrusion, slip-casting, tape-casting or spray-drying. In order to manufacture a high-quality support, a functionally gradient structure could be produced. Creating an asymmetric microstructure produces a smaller substrate pore size over a thinner region which results in superior permeation properties. ${ }^{13}$ Using tubular instead of flat supports would further increase the flux through the membrane due to the higher surface area per volume of the tubular support.

The quality of the support surface is of crucial importance to the integrity of the membrane. The homogeneity of the support will determine the integrity of the top-layers and the surface roughness determines the minimum thickness obtainable for complete coverage. ${ }^{7}$ Tubular supports can be produced by conventional methods such as extrusion or isostatic pressing followed by sintering, but these techniques are usually not suitable for porous membranes. The membranes manufactured in this manner often exhibit unroundness, surface roughness and an inhomogeneous microstructure. The roundness is very important in reactors, while unroundness would result in a radially inhomogeneous stress distribution in the tubes near the sealing, increasing the risk of brittle fracture. ${ }^{18}$

While an inhomogeneous packing of particles may result in a membrane with a higher permeability, such a membrane will have a lower tensile strength and a decreased surface smoothness. ${ }^{45}$ Using the more expensive method of centrifugal casting, an asymmetric support which offers low resistance to filtrate flow and delivers a smooth defect free inner-surface with adequate strength can be obtained. ${ }^{14}$

\subsection{Centrifugal casted ceramic membrane supports}

A cast can be obtained from a suspension of particles by the movement of particles through the liquid because of forces acting on the particles. This mechanism is known as sedimentation and is the foundation of cast formation in centrifugal casting. In this process, a powder is dispersed in a liquid with a stabilizing agent, after which the suspension is poured or injected into a mould-tube, which is placed in a horizontal or vertical centrifuge and rotated at typical rotation speeds from 
$15000-14000 \mathrm{rpm}$. After centrifugation the remaining liquid is poured or sucked out of the mould-tube containing the green compact. The resulting cast is dried, released from the mouldtube and sintered.

Although centrifugal casting is a rather new technique, numerous studies have been done on the theoretical and practical aspects concerning the suspension, the casting process and the sintering procedure in producing dense as well as porous ceramics supports. Ramzi et al. ${ }^{15}$ investigated the dispersion of $\mathrm{Al}_{2} \mathrm{O}_{3}$ suspensions, Tsetsekou et al. ${ }^{16}$ did work on the optimization of alumina slurries for ceramic processing applications and Santhiya et al. ${ }^{17}$ studied the surface chemistry of alumina suspensions using APMA. Biesheuvel et al. ${ }^{18}$ did theoretical modeling of the centrifugal casting process and Gogotsi et $a l^{8}$ modeled the solidification of functionally graded materials by centrifugal casting, while Zeng et al. ${ }^{19}$ and Darcovich et al. ${ }^{20}$ studied sintering aspects of alumina compounds.

\subsubsection{Colloidal suspension}

High quality ceramic membranes are produced by "colloidal" or "suspension processing" which suggests that an important step in the process is the dispersion of particles in a liquid and subsequent consolidation of the powder into a "green cast". Membranes produced by suspension processing result in ceramic products with superior properties because: $:^{21}$

- the particles can be dispersed effectively in the liquid phase by means of stirring, liquid milling and ultrasound

- homogeneous mixing of particles can take place

- centrifugation results in a close-packed structure, which is often uniform, defect free and has smooth surfaces

- due to the ordered packing and minimal amount of organic additives the drying and sintering failure and shrinkage can be minimized

Disadvantages of suspension processing are: ${ }^{21}$

- High cost

- Long processing times because of particle-liquid separation

- Undesired segregation due to gravity 
- Controlling the suspension state requires technical competence

The stability of the starting suspension is critical in the centrifugal manufacture process. If the starting suspension is too stable, the final sediment will remain fluid-like, so that an actual compact is not formed and redispersion occurs as soon as rotation ends. A less stable suspension on the other hand might give rise to flocculation of particles which would influence the homogeneity and surface roughness of the final compact. ${ }^{18}$ Polymers are usually used to stabilize particles in the suspension. The stabilizing effect largely depends on the nature of the side chains of the polymer. There are mainly three mechanisms by which a polymer can stabilize particles in a suspension namely: ${ }^{22}$

- Steric stabilization

- Electrostatic stabilization

- Depletion stabilization

Steric stabilization is a mechanism that can explain the ability of polymers to inhibit coagulation of suspensions. The polymers that are attached to the particles are believed to cover the system in such a way that long "loops" and "tails" extend out into the solution, thus creating dispersion of the particles due to this steric effect in the solution. Electrostatic stabilization occurs when the polymer contains negative or positive groups in its structure. By adjusting the $\mathrm{pH}$, either the negative or positive groups will be activated on the "tails" and "loops" extended in the solution, thus resulting in dispersion due to repulsive forces between the charged "tails" and "loops" of polymers attached to different particles in the solution. Depletion stabilization is provided by unanchored, unattached polymer molecules in the dispersion phase.

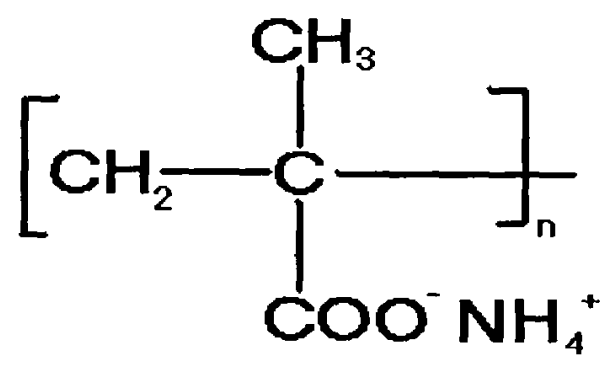

Figure 2.5 Structure of APMA. 
APMA (Ammonium PolyMethAcrylate aqueous solution) is a poly-electrolyte and is regularly used as a dispersant to stabilize ceramic particles in distilled water. ${ }^{17}$ The APMA structure is shown in Figure 2.5. According to Santhiya et al., ${ }^{17}$ hydrogen bonding, electrostatic and chemical interactive forces are responsible for the adsorption of APMA onto the surface of alumina powder in distilled water. The AMPA structure on the alumina surface stabilizes the particles in suspension by means of electrostatic and steric stabilization. ${ }^{22}$ Optimum stabilization is achieved in a ceramic suspension when a monolayer of the dispersant is absorbed onto the surface of the particles. ${ }^{23}$ The stabilizing effect further depends on the order in which the polymer is added, when the $\mathrm{pH}$ is adjusted and the temperature of the suspension. ${ }^{22}$

\subsubsection{Centrifugal casting}

Centrifugal casting is one of the methods of obtaining a green cast when making use of a suspension. Bieshevel., ${ }^{21}$ who did theoretical modeling on centrifugal casting, found that the primary difference between the suspension and a cast state is the yield stress, where the green cast by definition has a higher yield stress.

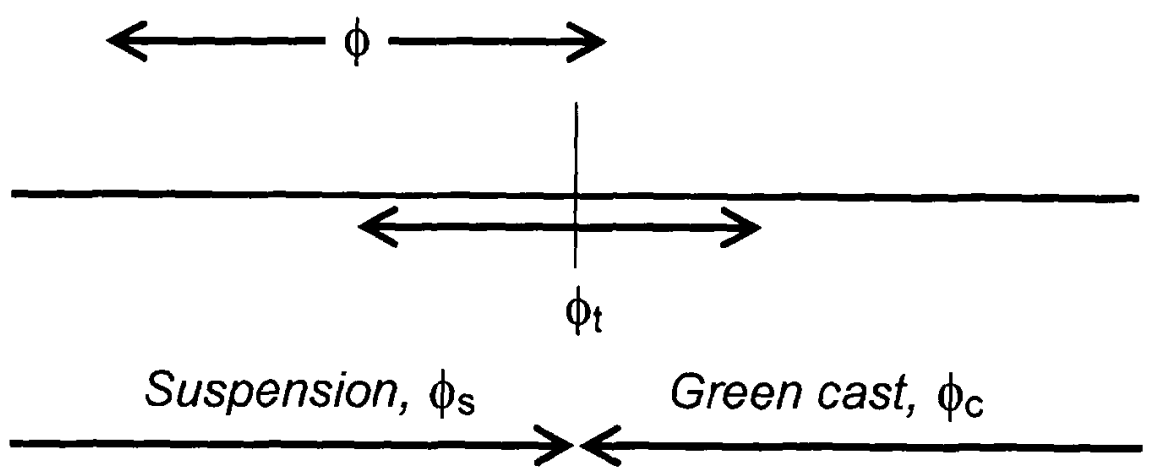

Figure 2.6 Yield stress of a suspension and the green casts.

In Figure 2.6, $\phi_{\mathrm{t}}$ is the transition concentration between suspension and cast, $\phi_{\mathrm{s}}$ is the suspension concentration, $\phi_{c}$ is the cast concentration and $\phi$ is the concentration of the suspension at the present time. In order to obtain a cast state from a suspension, $\phi$ has to be larger than $\phi_{\mathrm{t}}$. This can be achieved by either decreasing $\phi_{\mathrm{s}}$ or increasing $\phi$. In a process like centrifugal casting $\phi_{\mathrm{s}}$ is a set parameter and thus $\phi$ has to be increased. This can be obtained by exerting an external 
force onto the particles which will result in a cast formation. The use of a high centrifugal force on the particles will result in a graded membrane. In centrifugal casting, the largest particles move firstly to the mould wall, followed by the smaller particles. This is because centrifugal casting makes use of gravitational potential. The velocity at which particles move outwards towards the mould-wall depends on their mass. ${ }^{21}$ After centrifugal casting the cast usually has a porosity between 25 and 60 volume $\%{ }^{32}$

\subsubsection{Sintering}

Sintering at elevated temperatures is the next step in the production of a porous ceramic support for application in membrane technology. Strengthening via sintering is a necessity, but a decrease in the permeability (porosity) is not desirable. A change in pore shape, a decrease in surface area and porosity and an increase in neck area and grain size (grain growth) occurs during sintering of the cast. According to Kingery et al. ${ }_{1}{ }^{24}$ grain growth is the process by which the average grain size of strainfree or nearly strainfree material increases continuously during heat treatment without change in grain-size distribution. There are various mechanisms during sintering that takes place as shown in Table 2.2. ${ }^{32}$

Table $2.2 \quad$ Sintering mechanisms

\begin{tabular}{cc}
\hline Mechanism (number) & Source of matter \\
\hline Surface diffusion (1) & Surface \\
Lattice diffusion (2) & Surface or grain boundary \\
Vapour transport (3) & Surface \\
Boundary diffusion (4) & Grain boundary \\
\hline
\end{tabular}




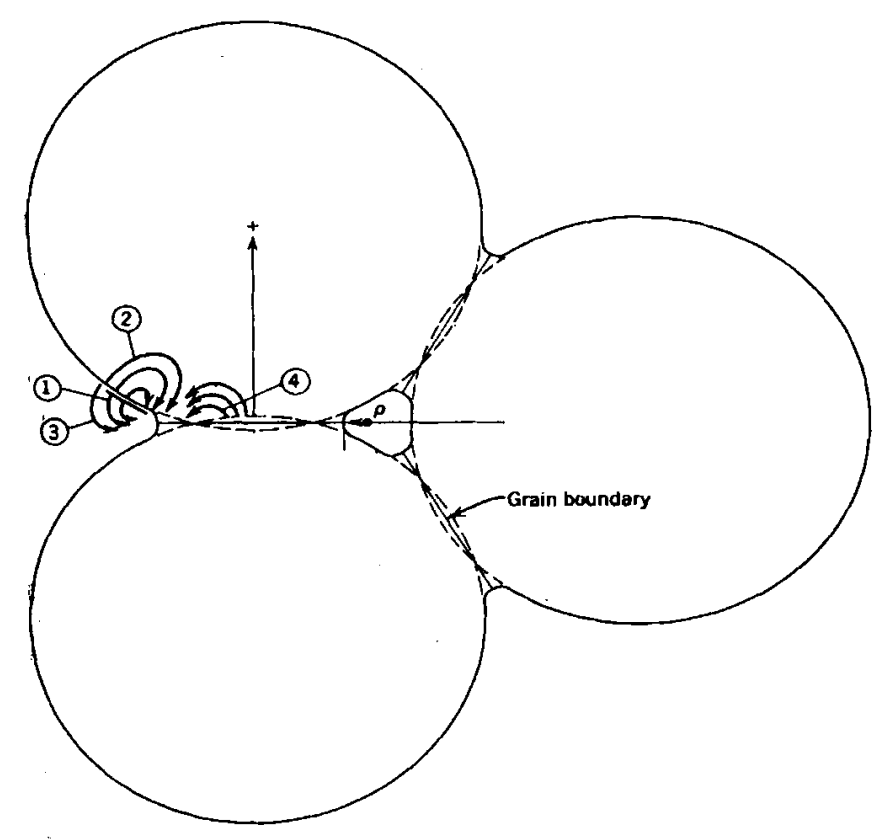

Figure 2.7 Mechanisms occurring during sintering.

The difference in free energy or chemical potential between the neck area and the particle surface in a compact provides a driving force which causes the transfer of material. The sintering mechanisms mentioned in Table 2.2 are illustrated in Figure 2.7. Mechanism number 3 illustrates vapor transport which occurs a fair distance from the grain boundary. If the vapor pressure is low, material transfer may occur by solid-state processes like surface, lattice or grain boundary diffusion. Which one or more of these processes actually contribute significantly depends on which mechanism results in the most rapid decrease in the free energy of the system. The most significant difference between these mechanisms is that the transfer of material between the neck and the surface of the particles (surface diffusion, lattice diffusion and vapor transport) does not lead to shrinkage of the compact while material transfer from the particle volume or the grain boundary (boundary diffusion) causes shrinkage and pore elimination. ${ }^{32}$

Determination of the sintering kinetics has been successful making use of the linear shrinkage rate. $^{19}$ The rate of sintering influences the final product. The rate of shrinkage and densification is higher if a low rate of sintering is used. This is can be achieved by using a low heating rate ${ }^{25}$ The driving force for densification is the change in free-energy. This change in free-energy is caused by a decrease in the surface area and the lowering of the surface free-energy by the elimination of solid-vapor interfaces. 
The effect of sintering temperature on powder compacts has been widely studied, but the results obtained by various scientists differ. Hillman et al. ${ }^{26}$ stated that the mean pore size increases with increasing sintering temperature. Page et al. ${ }^{27}$ found that the pore size remains constant while Fang et al. ${ }^{28}$ observed that the transition of the pore size during sintering depends on the porosity of the green compacts. Wang et al. ${ }^{28}$ produced tubular membranes by means of slip-casting and found that the pore size of the supports increased with sintering temperature, while the pore size distribution remained constant. They also observed that the thicker the membranes, the smaller the pores. ${ }^{28}$ Steenkamp et $a l .{ }^{32}$ found that the porosity and the pore radius decreased with increased sintering temperature.

The particle size of the starting powder also has an effect on the properties of the support as observed by various authors such as Steenkamp et al. ${ }^{32}$ and Wang et al. ${ }^{28}$ The smaller the particle size, the smaller the pores and the lower the porosity and water permeability of the support at a given sintering temperature.

There are various methods available for sintering ceramic compacts in order to reduce or enhance grain growth depending on the specific application of the membrane manufactured. Nijmeijer et al. ${ }^{14}$ prepared porous tubular membranes by conventional sintering in an oven, Zhou et al. ${ }^{29}$ studied the effect of sintering rate on particle size by implementing pulse electric current sintering (PECS). By sintering with PECS, very high sintering rates (up to $300^{\circ} \mathrm{C} / \mathrm{min}$ ) can be obtained. Microwave sintering is also often used for rapid heating and manufacturing of ceramics with improved microstructures. Mizunoa et $a l^{30}$ investigated the sintering of alumina slips produced by microwave sintering. With this method they studied sintering rates up to $15^{\circ} \mathrm{C} / \mathrm{min}$ from room temperature to $1500^{\circ} \mathrm{C}$. Another method of sintering is Selective Laser Sintering (SLS) which is mainly used to manufacture complex ceramic structures or forms, but this method is not currently used in membrane applications. ${ }^{31}$ 


\subsection{Characterization of membranes}

Once a membrane has been manufactured it needs to be characterized in order to determine the suitability of the membrane for a specific application. Through characterization, the membrane's structural and morphological properties are determined, which determine its functionality. Since the inner or top-layer of a membrane is responsible for its separation properties, the rejection or separation factor is used to characterize this part of the membrane. Pervaporation, gas separation or liquid permeation are a few of the techniques employed to determine rejection and separation. ${ }^{1}$

Various methods are available to obtain structural information on the support. Some of the important properties of the support structure are permeability, porosity, pore diameter, pore size distribution, stability, surface quality and structural strength.

\subsubsection{Permeability}

Characterization by permeability is often used due to its simplicity and availability. In order to obtain permeation data for a ceramic support, a specific pressure difference is applied over the membrane and the quantity of liquid, for example water, permeating through the membrane per time unit is measured. The water permeation can be calculated by determining the water flux through the membrane at various pressures to obtain a graph of water flux against pressure difference. Dividing the slope of the straight line by the surface area of the membrane, the water permeability can be calculated. ${ }^{1}$ The permeability can also be used to characterize the type of membrane process involved. Table 2.3 gives a summary of the pressure and flux ranges for various processes. ${ }^{1}$ 
Table 2.3 Pressure and flux ranges for various membrane processes ${ }^{1}$

\begin{tabular}{ccc}
\hline Membrane process & Pressure range (Bar) & Flux range $\left(\mathbf{~ m}^{-2} h^{-1}\right.$ bar $\left.^{-1}\right)$ \\
\hline Microfiltration & $0.1-2.0$ & $>50$ \\
Ultrafiltration & $1.0-5.0$ & $10-50$ \\
Nanofiltration & $5.0-20.0$ & $1.4-12$ \\
Reverse osmosis & $10-100$ & $0.05-1.4$ \\
\hline
\end{tabular}

The water permeability of a porous support is influenced by various factors such as the particle size of the starting powder, the temperature at which the support was sintered ${ }^{1}$ and the amount of dispersant used in stabilizing the colloidal suspension. ${ }^{2}$ This is due to the influence of the above mentioned factors on the porosity, pore radius and mechanical strength of the support structure. ${ }^{32}$ This effect can be visualized by the extended Hagen-Poisseule equation

$$
J=\frac{\varepsilon}{\tau} \frac{r^{2}}{8 \eta} \frac{\Delta P}{\Delta x}
$$

where $\mathrm{J}$ is the flux $\left(\mathrm{L} . \mathrm{h}^{-1} \mathrm{~m}^{-2}\right)$ through the membrane at a driving force $\Delta \mathrm{P} / \Delta \mathrm{x}$, with $\Delta \mathrm{P}$ the pressure difference $\left(\mathrm{N} / \mathrm{m}^{2}\right)$ and $\Delta x$ the membrane thickness $(\mathrm{m}) . \varepsilon$ is the porosity $(-), \eta$ is the viscosity (Pa.s), $\tau$ is the tortuosity $(-)$ and $r$ is the median pore radius $(m)$. For spherical particles $\tau$ is equal to 2.5 . The unit frequently used in literature for permeability is $L . m^{-2} \cdot h^{-1} \cdot b a r^{-1}$ (32, 33) The HagenPoiseuille equation is applied on the assumption that the median pore radius as well as porosity remain constant throughout the porous structure of the membrane, which is not always accurate when calculating porosity or pore radius in a membrane where a porosity gradient exists through the thickness of the membrane.

\subsubsection{Pore size and porosity}

Obtaining structural information like pore size, pore size distribution and porosity of the structure is important as it has an effect on the properties of the membrane. A characterization technique like mercury intrusion porosimetry is suitable for obtaining this structural information. In mercury intrusion porosimetry, non-wetting mercury is used to gain information on the porous structure of 
solid materials. Washburn ${ }^{1}$ proposed in 1921 that mercury can be injected into porous materials to determine pore size distributions. This proposal led to the Washburn equation:

$$
\Delta P=\frac{4 y \cos \theta}{d}
$$

where $\gamma$ is the surface tension of mercury $(0.48 \mathrm{~N} / \mathrm{m}), \theta$ the contact angle of mercury on the material being intruded $\left(141.3^{\circ}\right), \mathrm{P}$ is the pressure required to force a non-wetting liquid into the pores $(\mathrm{Pa})$ and $d$ is the diameter of the pores $(m)$. Mercury is forced into a dry membrane at various pressures with the volume of mercury intruded determined at each pressure.

The pore size distribution of the sample can be estimated in terms of the volume mercury intruded for a given diameter. The calculated pore diameter at an intrusion pressure $P$ depends on the assumption made for the pore geometry, which is taken as cylindrical. Larger pores that are only accessible by smaller pores will incorrectly be counted as small pores. This is known as the inkbottle pore effect, illustrated in Figure 2.8 .
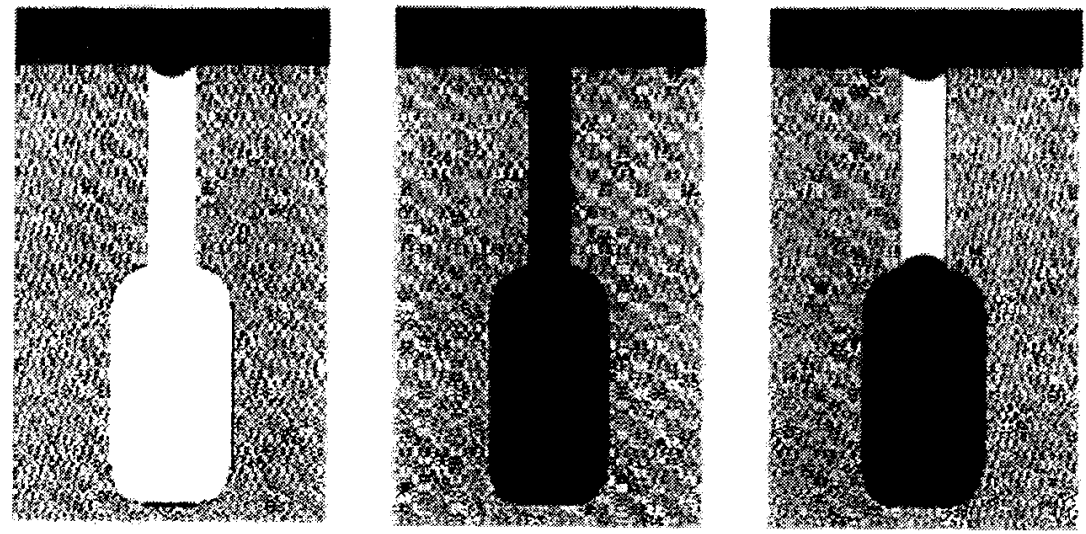

Figure 2.8 The ink-bottle effect. The mercury intrudes at the given pressure of the small pore, in this example the large pore connected to the smaller pore is counted as a small pore.

The ink-bottle effect and the inability of mercury intrusion to differentiate between pore types makes this method less accurate when information concerning the permeation properties of a membrane has to be obtained using structural information such as pore size and pore size 
distribution. Mercury porosimetry includes closed and blind pores into the pore size and pore size distribution of the membrane structure, but only the open pores determine the permeability of the membrane. ${ }^{6}$ Jena et $a l^{6}$ made use of capillary flow porometry with modifications in design to measure the throat diameter of pores, the largest pore throat diameter, the mean pore diameter, the pore size distribution and the permeability of a porous ceramic tube.

\subsubsection{Chemical and thermal stability}

The stability of a membrane is important because it influences the life-time and thus the economic value of a membrane in a specific application. If a membrane's chemical stability is limited with respect to corrosive media like strong acids and organic solvents, or its thermal stability is influenced by temperature changes, then the effective separation applications of the membrane will be restricted. ${ }^{34}$

The chemical stability is defined as the inertness against aggressive liquids and could be determined by permeating a specific liquid through the membrane. By comparing structural (separation) and permeation properties before and after permeation, the chemical stability of the membrane for the specific liquid can be established. Pore-stability, stability of particle sizes of the membrane or stability of the crystallographic phases in the membrane can be defined as the temperature stability. ${ }^{34}$

\subsubsection{Membrane surface}

Membrane fouling increases operational and maintenance costs in industries where membrane processes are used. ${ }^{35}$ An important aspect of membrane fouling is the contribution of the membrane surface and therefore it is important to study the chemical and physical properties of the membrane surface.

In composite membranes where a selective layer like a zeolite is deposited onto a porous support, it is important to study the surface, because aspects such as surface roughness and chemical properties of the surface could determine whether the separation layer attaches to the support structure. In literature, authors have used various methods to make the support surface more accessible for attachment for example by drying the support, immersion in a specific fluid, ${ }^{36}$ positive charging of the membrane surface ${ }^{37}$ creating oxygen groups on the surface ${ }^{38}$ and 
cleaning of the support surface by ultra-sound. ${ }^{39}$ The methods differ depending on the support and separation layer properties and the synthesis technique employed.

As mentioned earlier, the surface roughness also has an influence on the thickness of the separation layer and thus indirectly on the rate of flux through the composite membrane. Recent studies have shown that surface structure and morphology influence the performance of membranes. ${ }^{40,41,42}$ It has even been shown that there is a relationship between surface roughness and flux. ${ }^{42}$ Properties such as pore size, pore size distribution, surface roughness, chemical properties (hydrophobic/hydrophilic) and electrokinetic characteristics (zeta-potential) could be investigated to determine whether the top-layers could be deposited successfully onto the support surface. ${ }^{42}$

Several analytical techniques are available to study characteristics of the membrane surface. These techniques include Raman spectroscopy, NMR, X-ray photoelectron spectroscopy(XPS), ${ }^{43}$ Scanning Electron Microscopy (SEM) and Atomic Force Microscopy (AFM). SEM is a very simple and useful technique yielding a clear picture of the top-layer, cross section or bottom layer of a membrane (Figure 2.9).

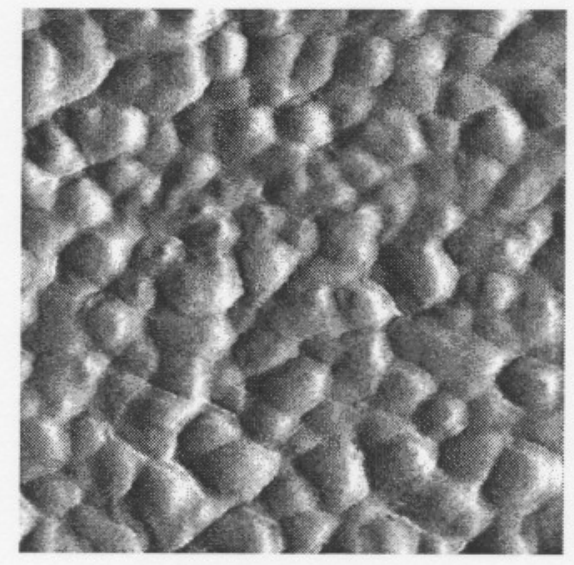

Figure 2.9 SEM image of a ceramic membrane surface.

Although a rough estimation of the porosity and pore radius can be obtained by using a SEM photograph, the use of SEM to acquire a visual perception of the surface roughness and possible defects in or on the surface of a membrane is more common.

The scanning electron microscope (SEM) is a microscope that uses electrons rather than light to form a high resolution image of an object. Higher magnification, larger depth of focus, greater resolution and ease of sample observation makes SEM one of the most used instruments in 
research today. The principal is as follows: Electrons are emitted from an electron source and accelerated towards an anode and down a column. The beam of electrons is condensed by a lens and focused as a very fine point onto the sample. When the electrons strike the sample, both electron and proton signals are emitted. The signals most commonly used are the secondary electrons and backscattered electrons. In order to obtain the signals induced by electron bombardment, the sample needs to be conductive. Non-metallic samples such as bugs, plants and ceramics therefore have to be coated with, for example, gold or platinum. ${ }^{44}$

Atomic force microscopy (AFM) is a more novel method to characterize membrane surfaces. Pore size and porosity of the membrane surface structure can be obtained from cross-section images. The advantage of this technique is that no membrane pretreatment is required, but disadvantages are that very rough surfaces may be difficult to interpret and high forces may damage polymeric structures. ${ }^{1}$ Figure 2.10 shows a typical AFM image.

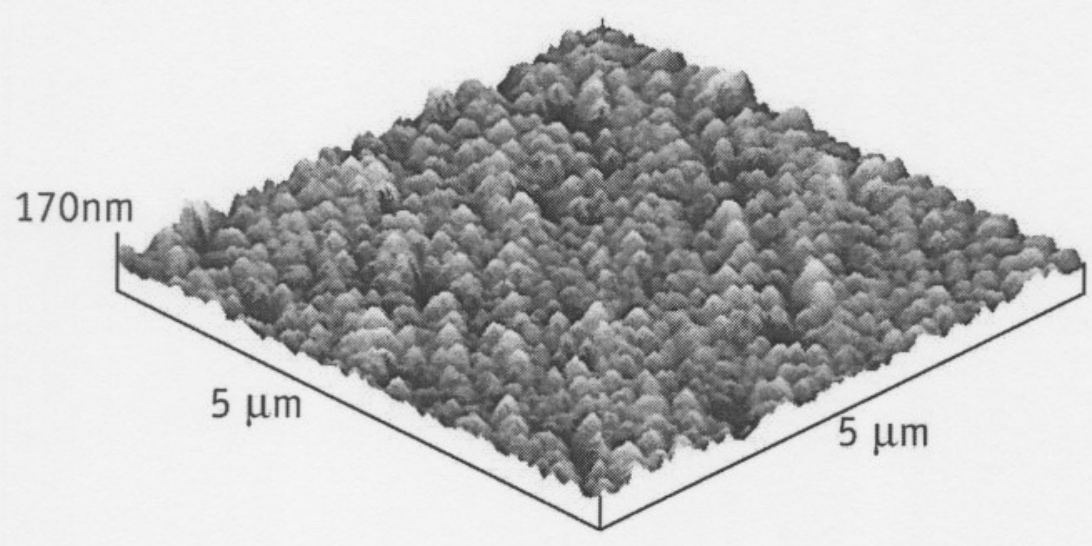

Figure 2.9 An AFM image of the sample shown in Figure 2.9.

\subsubsection{Strength}

To ensure that a ceramic membrane support has enough strength to endure high pressure differences or extreme conditions that could be present in a separation process, the strength of the support structure should be investigated.

The tensile strength depends on the porosity and the size of the largest flaw type present. Thus macrodefects such as the extent of aggregate-formation prior to sintering would affect the tensile strength. To obtain maximum tensile strength, all agglomerates have to be removed from the 
suspension from which the membranes is being made and clean-room facilities have to be used to ensure that a homogeneous ceramic is produced. ${ }^{45}$

A universally accepted method to evaluate the fracture toughness (strength) of structural ceramics is yet to emerge in material science. In literature, various researchers have adopted different techniques to evaluate the fracture toughness of a ceramic structure. This is since no particular method has been given preference as each of them has appreciable shortcomings restricting their application. Techniques employed include the miniaturized disk bend test when small amounts of material is available, ${ }^{46}$ the measurement of crack opening displacement, ${ }^{47}$ the single edge notched beam applied under 3 point loading, ${ }^{48}$ the compact tension method, ${ }^{49}$ the indentation technique ${ }^{50}$ and the scratch test.

Although all these techniques are useful, the data obtained are not always comparable. When evaluating the tensile strength of tubular supports, even fewer techniques are available. Diametral compression testing, ${ }^{51}$ the four-point-bending-test ${ }^{45}$ and the three-point-bending-test ${ }^{32}$ are some of the methods used for tubular structure strength evaluation.

Steenkamp et al. $^{32}$ investigated the fracture strength of centrifugal casted ceramic supports by means of the tree-point-bending test. No literature was found on strength testing from the inside of a tubular ceramic structure. Biesheuvel et al., ${ }^{18}$ however, noted that centrifugal casted supports withstand 50 bar of water pressure from the inside before breakage occurred.

\subsection{Conclusion}

Separation by means of membrane technologies is likely to play an increasingly important role in industry as environmental impact and costs of separation processes rise.

For industrial separation applications, inorganic membranes as an alternative to polymeric membranes could be considered due to their high thermal, chemical, and mechanical stability. Inorganic membranes are usually multi-layered systems, consisting of a support and one or more top-layers. The support provides the mechanical strength, whilst the top-layers are responsible for the separation properties of the membrane. The optimization of the composite membrane is achieved by increasing the porosity of the support whilst decreasing the thickness of the top-layers.

The homogeneity and the surface roughness of the support determine the integrity and minimal thickness of the top-layer, while the use of tubular instead of flat supports would further increase the flux through the membrane due to the higher surface area per volume of the tubular support. 
The centrifugal casting method can be used to obtain an asymmetric tubular support, which offers low resistance to filtrate flow and delivers a smooth defect free inner-surface with adequate strength. As strength is critical for operating under extreme mechanical and thermal conditions, the use of a ceramic structure as a support is a major advantage.

High quality ceramic membranes are produced by "colloidal" or "suspension processing". Optimization of the support structure can be achieved by optimizing the suspension characteristics and sintering conditions during the manufacturing procedure.

Once the membrane has been manufactured, it needs to be characterized in order to determine the suitability of the membrane for a specific application. Structural information on the support can be obtained by determining permeability, pore size, porosity, stability and mechanical strength while the surface integrity and smoothness can be investigated by means of scanning electron microscopy.

Optimization of the support structure is necessary in obtaining a composite membrane which would deliver optimum results for a specific application. By investigating the suspension properties and the sintering variables present during the manufacturing procedure of the support structure, the optimal conditions can be predicted. 


\subsection{References}

1 M.Mulder, Basic principles of membrane technology, second edition, Kluwer Academic Publishers, 1996, Chapter 1: Introduction, pp. 7

2 J.M.Benito, A.Conesa, F.Rubio, M.A.Rodriguez, Preparation and characterazation of tubular ceramic membranes for treatment of oil emulsions, Journal of the European Ceramic Society, 25, 2004, 1895

${ }^{3}$ T.A.Peters, J.Fontalvo, M.A.G.Vorstman, N.E.Benes, R.A. van Dam, Z.A.E.P.Vroon, E.L.J. van Soest-Vercammen, J.T.F.Keurentjes, Hollow fibre microporous silica membranes for gas separation and pervaporation sythesis, performance and stability, Journal of Membrane Science, 248, 2005, 73

${ }^{4}$ G.T.P.Mabande, G.Pradhan, W.Schwieger, M.Hanebuth, R.Dittmeyer, T.Selvam, A.Zampieri, H.Baser, R.Herrmann, A study of Silicalite-1 and A1-ZSM-5 membrane synthesis on stainless steel supports, Microporous and Mesoporous Materials, 75, 2004, 209

${ }^{5}$ R.M. de Vos, H.Verweij, High-selectivity, high-flux silica membranes for gas separation, Journal of Membrane Science, 279, 1998, 1710

${ }^{6}$ A.Jena, K.Gupta, Porosity characterization of microporous small ceramic components, Porous Materials Inc., 2001

7 P.M.Biesheuvel, V.Breedveld, A.P.Higler and H.Verweij, Graded membrane supports produced by centrifugal casting of a slightly polydisperse suspension, Chemical Engineering Science, 56(11), 2001,3517

${ }^{8}$ G.A.Gogotsi, Fracture toughness of ceramic and ceramic composites, Ceramics International, 29, 2003,777 
9 J.Caro, M.Noack, P.Kölsch, R.Schäfer, Zeolite membranes - state of their development and perspective, Microporous and Mesoporous Materials, 38, 1999, 3

${ }^{10}$ D.Jayaseelan, S.Ueno, T.Ohji, S.Kanzaki, Thermo-mechanical stability of porous alumina:effect of sintering parameters, Science and Technology of Advanced Materials, 5, 2004, 387

11 T.Isobe, T.Tomita, Y.Kameshima, A.Nakajima, K.Okada, Preparation and properties of porous alumina ceramics with oriented cylindrical pores produced by an extrusion method, Journal of the European Ceramic Society, Article in Press, 2005

${ }^{12}$ K.Darcovich, L.Béra, K.Shinagawa, Particle size distribution effects in an FEM model of sintering porous ceramics, Material Science and Engineering, A341, 2002, 247

${ }^{13}$ K.Darcovich, L.Béra, K.Shinagawa, Particle size distribution effects in an FEM model of sintering porous ceramics, Material Science and Engineering, A341, 2002, 247

${ }^{14}$ A.Nijmeijer, C.Huiskes, N.G.M.Sibelt, H.Kruidhof and H.Verweij, Centrifugal casting of tubular membrane supports by centrifugal casting, American Ceramic Society Bulletin, 77, 1998, 95

15 M.Ramzi, B.Romdhane, S.Boufi, S.Baklouti, T.Chartier, J.Baumard, Dispersion of $\mathrm{Al}_{2} \mathrm{O}_{3}$ suspension with acrylic copolymers bearing carboxylic groups, Colloids and Surfaces A, 212, 2003, 271

${ }^{16}$ A.Tsetsekou, C.Agrafiotis, A.Milias, Optimization of the rheological properties of alumina slurries for ceramic processing applications; Journal of the. European Ceramic Society, 21, 2001, 363

${ }^{17}$ D.Santhiya, S.Subramanian, K.A.Natarajan, S.G.Malghan, Surface chemical studies on alumina suspension using ammonium poly(methacrylate), Colloids and Surfaces A, 164, 2000, 143 
${ }^{18}$ P.M.Biesheuvel, A.Nijmeijer and H.Verweij, Theory of batchwise centrifugal casting, AlChE Journal, 44, 1914-1922

${ }^{19}$ W.Zeng, L.Goa, L.Gui, J.Guo, Sintering kinetics of $\alpha-\mathrm{Al}_{2} \mathrm{O}_{3}$, Ceramics International, 25, 1999, 723

${ }^{20}$ K.Darcovich, K.Shinagawa, F.Walkowiak, A three-dimensional dual-mechanism model of pore stability in a sintering alumina structure, Material Science and Engineering A, 373, 2004, 107

21 P.M.Biesheuvel, Ph.D. Thesis, Universiteit Twente, The Nederlands, 1999, Porous ceramic membranes suspension processing, mechanical and transport properties, and application in the osmotic tensiometer, Summary

${ }^{22}$ T.P.Raming, Universiteit Twente, The Nederlands, 1996, The stability of colloidal suspensions

${ }^{23}$ A.Zupancic, R. Lapasin, A.Kristoffersson, Influence of Particle concentration on Rheological properties of Aqueous $\alpha-\mathrm{Al}_{2} \mathrm{O}_{3}$ suspension, Journal of the European Ceramic Society, 18, 1998, 467

${ }^{24}$ W.D.Kingery, H.K. Bowen, D.R.Uhlmann, Introduction to ceramics, Second Edition, Chapter 10, 1976

${ }^{25}$ J.Opfermann, J.Blumm, W.D. Emmerich, Simulation of the sintering behavior of a ceramic green body using advanced thermokinetic analysis, Thermochimica Acta, 318, 1998, 213

${ }^{26}$ S.H.Hillman, R.M.German, Constant heating rate analysis of simultaneous sintering mechanics in alumina, Journal of Material Science, 27,1992, 2641

${ }^{27}$ R.A.Page, Y.M.Pan, Microstructure evolution during sintering, Material Research Society, Symp. Proc., 249, 1992, 449 
${ }^{28}$ P.Wang, P.Huanga, N.Xua, J.Shia, Y.S.Linb, Effects of sintering on properties of alumina microfiltration membranes, Journal of Membrane Science, 155, 1999, 309

${ }^{29}$ Y.Zhou, K.Hirao, Y.Yamauchi, S.Kanzaki, Effects of heating rate and particle size on pulse electric current sintering of alumina, Scripta Materialia, 48, 2003, 1631

${ }^{30}$ M.Mizunoa, S.Obataa, S.Takayamaa, S.Itoa, N.Katoa, T.Hiraia, M.Satob, Sintering of alumina by $2.45 \mathrm{GHz}$ microwave heating, Journal of the European Ceramic Society, 24, 2004, 387

${ }^{31}$ D.Bourell, M.Wohlert, N.Harlan, S.Das, J.Beman, Powder densification maps in selective laser sintering, Advanced Engineering Materia/s, 4(9), 2002, 663

${ }^{32}$ G.C.Steenkamp, A.Nijmeijer, H.M.Krieg, K.Keizer, Centrifugal casting of ceramic membrane tubes and coating with chitosan, Separation and Purification Tegnology, 25, 2001, 407

${ }^{33}$ K.Kim, S.Cho, K.Yoon, J.Kim, J.Ha, D.Chun, Centrifugal casting of alumina tube for membrane application, Journal of Membrane Science, 199, 2002, 69

34 J.Hofman-Züter, Ph.d. Thesis, University of Twente, Nederlands, 1995, Chemical and thermal stability of (modified) mesoporous ceramic membranes, Chapter 2, Theoretical background, 9-52

${ }^{35}$ E.M.Vrijenhoek, S.Hong, M.Elimelech, Influence of membrane surface properties on initial rate of colloidal fouling of reverse osmosis and nanofiltration membranes, Journal of Membrane Science, 188, 2001, 115

${ }^{36}$ J.Li, Q.T.Nguyen, L.Z.Zhou, T.Wang, Y.C.Long, Z.H.Ping, Preparation and properties of ZSM-5 zeolite membrane obtained by low-temperature chemical vapor deposition, Desalination, 147, 2002,321 
${ }^{37}$ J.Hedlund, M.Noack, P.Kölsh, D.Creaser, J.Caro, J.Sterte, ZSM-5 membranes synthesized without organic templates using seeding technique, Journal of Membrane Science, 159, 1999, 263

38 A.Berenguer-Murcia, J.Garcia-Martinez, D.Cazorla-Amoros, A.Linares-Solano, A.B.Fuertes, Silicalite-1 membranes supported on porous carbon discs, Microporous and Mesoporous Materials, 59, 2003, 147

${ }^{39}$ Z.A.E.P.Vroon, K.Keizer, A.J.Burggraaf, H.Verweij, Preparation and characterization of thin zeolite MFI membranes on porous supports, Journal of Membrane Science, 144, 1998, 65

${ }^{40}$ K.Riedl, B.Girard, R.W.Lencki, Influence of membrane structure on fouling layer morphology during apple juice clarification, Journal of Membrane Science, 139, 1998, 155

${ }^{41}$ M.Elimelech, X.Zhu, A.E.Childress, S.Hong, Role of membrane surface morphology in colloidal fouling of cellulose acetate and composite aromatic polyamide reverse osmosis membranes, Journal of Membrane Science, 127, 1997, 101

${ }^{42}$ M. Hirose, H.Ito, Y.Kamiyama, Effect of skin layer surface structures on the flux behaviour of RO membranes, Journal of Membrane Science, 121, 1996, 209.

${ }^{43}$ E.Ferjani, M.Mejdoub, M.S.Roudesli, M.M.Chehimi, D.Picard, M.Delamar, XPS characterization of poly(methylhydrosiloxane)-modified cellulose diacetate membranes, Journal of Membrane Science., 165, 2000, 125

${ }^{44}$ www.unl.edu/CMRAcfem/semoptic.htm.

${ }^{45}$ P.Maarten Biesheuvel, Henk Verweij, Design of ceramic membrane supports: tensile strength and stress, Journal of Membrane Science, 156, 1999, 141 
${ }^{46}$ F.C.Chen, A.J.Ardell, Fracture toughness of ceramics and semi-brittle alloys using a miniaturized disk-bend test, Innovations in Material Research, 1, 1996, 47

${ }^{47}$ A.S.F.Haubensak, A New Method of Fracture-toughness determination in brittle ceramics by Open-Crack-Shape-Analysis, Journal of Material Science., 32, 1997, 1473

48 S.Gautier, E.Champion, D.B.Assollat, Rheological characteristics of alumina platelethydroxyapatite composite suspensions, Journal of the European Ceramic Society, 17, 1997, 1361

${ }^{49}$ C.Anja, Journal of Material Science, 16, 1997, 1300

${ }^{50}$ M.Guazzato, M.Albakry, S.P.Ringer, M.V.Swain, Strength, fracture toughness and microstructure of a selection of all-ceramic materials, Part I. Pressable and alumina glass-infiltrated ceramics, Dental Materials, 20, 2004, 441

${ }^{51}$ C.H.Chen, K.Takita, S.Honda, H.Awaji, Fracture behavior of cylindrical porous alumina with pore gradient, Journal of the European Ceramic Society, 25, 2005, 385 


\section{Chapter 3}

\section{Optimization of \\ tubular ceramic}

\section{membrane}

supports

\subsection{Introduction}

While polymeric membranes are suitable for water related applications, many separation processes in industry require a membrane with high temperature and chemical stability as well as sufficient strength to endure aggressive environments. ${ }^{1,2}$ For this purpose, inorganic membranes are suitable due to their thermal, chemical and mechanical stability. ${ }^{3}$ To obtain a higher flux and thus a more efficient separation, tubular instead of flat membranes are recommended. ${ }^{4}$ Tubular membranes with high permeability can be obtained by producing a composite membrane consisting of a macroporous support and a dense inner membrane layer. The support made from ceramic material, for example, provides the mechanical strength on which a top-layer can be synthesized. The top-layer, for example a zeolite, has a specific pore size and chemical properties 
to ensure the required separation of specific mixtures. While ceramic materials commonly used for the production of membrane supports include alumina, silica, titania and zirconia, ${ }^{3}$ the low cost and abundance of alumina makes it an ideal material for the commercial manufacture of inorganic supports. Since alumina powder is available in highly purified grades and wide ranges of particle sizes, it is ideally suited for fundamental studies in material research. ${ }^{2}$ Although inorganic membrane manufacture is more expensive than polymeric membrane manufacture, the long term cost implications due to their chemical and thermal stability over a period of time make inorganic membranes a viable option.

Ceramic supports have a few advantages when compared to stainless steel supports. In order to obtain a maximum flux through a composite membrane, the top-layer has to be as thin as possible. For this reason a smooth membrane support surface is required. In general, the surface of stainless steel supports is rougher than the surface of a ceramic support. ${ }^{5}$ The other disadvantage is that stainless steel supports have a higher thermal expansion coeffisient $\sim 15-19 \times 10^{-6} \mathrm{~K}^{-1}$ compared to the $-2-7 \times 10^{-6} \mathrm{~K}^{-1}$ for alumina. During calcination the zeolite top-layer shrinks due to template burn-off. Since the expansion coeffisient difference between the zeolite and the stainless steel is higher than the difference between the zeolite and the ceramic support, the stainless-steelsupported zeolite tends to be more prone to thermal cracking and adhesion problems. In the view of the above mentioned problems, it is thus more difficult to produce a thin, defect free zeolite membrane directly onto a porous stainless steel support. ${ }^{6}$

Although ceramics and ceramic composites have rather high strength characteristics, they tend to have low crack resistance. The preparation of porous ceramic membranes is furthermore expensive due to the cost of their high purity powder and the techniques employed to manufacture a novel product. These are two of the more important obstacles hindering the wide-scale use of these materials for membrane applications. ${ }^{7,8}$

Producing tubular ceramic supports by conventional methods like extrusion or isostatic pressing followed by sintering are acceptable techniques, but unroundness, insufficient microstructural homogeneity and considerable surface roughness may impose problems in the application of these tubes. Centrifugal deposition of a colloidal suspension is a more expensive method but is suitable for the manufacturing of high quality tubes with a homogenous packing of particles and a smooth inside surface, ideally suited for the deposition of a thin, defect free top-layer.

It is the aim of this study to prepare centrifugal casted supports using three different powder sizes at various sintering temperatures and to investigate the effect of the particle size and sintering temperature on the properties of the supports such as water permeability, porosity, pore size and structural strength. 


\subsection{Experimental}

\subsubsection{Effect of sintering temperature and powder size}

For this study AKP-50, AKP-30 and AKP-15 (Sumitomo Chemical Company, Ltd., Japan) powders were used to make $\alpha-\mathrm{Al}_{2} \mathrm{O}_{3}$ supports. According to the manufacturer, the AKP-30 powder has a mean particle size of $0.31 \mu \mathrm{m}$ and a BET surface area of $6.9 \mathrm{~m}^{2} \mathrm{~g}^{-1}$, while the AKP-15 powder has a mean particle size of $0.61 \mu \mathrm{m}$ and a BET surface area of $3.4 \mathrm{~m}^{2} \mathrm{~g}^{-1}$. Both powders have a chemical purity of $>99.99 \%$ and a narrow particles size distribution. According to the supplier, the AKP-50 powder has a BET surface area of $10.4 \mathrm{~m}^{2} \mathrm{~g}^{-1}$, but no mean particle diameter was given. According to literature the mean particle size of the AKP-50 powder is between $0.1-0.3 \mu \mathrm{m}$ with a mean particle size average of $0.25 \mu \mathrm{m}^{9}$

To obtain three membranes with a length of $6 \mathrm{~cm}$ and a inner diameter of $20 \mathrm{~mm}$ each, $42 \mathrm{~g}$ of alumina powder was suspended in a mixture of $49 \mathrm{~g}$ deionised water and $10 \mathrm{~g}$ APMA (Ammonium PolyMethAcrylate aqueous solution, Darvan C, R.T. Vanderbilt Company, Inc., Norwalk, USA). Before adding the powder to the water/polymer mixture, the $\mathrm{pH}$ of the mixture was brought to 9.5 using a $\mathrm{NH}_{4} \mathrm{OH}$ solution. The suspension was ultrasonically treated for 10 minutes at a frequency of $20 \mathrm{kHz}$ and a transducer output of 100W (Model 250 Sonifier, Branson Ultrasonics Corporation, Danbury, USA). The well dispersed suspension was poured through a sieve with apertures of $106 \mu \mathrm{m}$ to remove any large agglomerates. The suspension was subsequently stirred for 1 hour at $30^{\circ} \mathrm{C}$ to obtain a homogeneous suspension, before it was poured into steel moulds for centrifugation. The inside wall of the steel moulds were pre-coated with a solution of Vaseline and petroleum ether (boiling range $60-80^{\circ} \mathrm{C}$ ) to ensure easy mould release.

The tubes were centrifuged for 10 minutes at $17000 \mathrm{rpm}$ using a custom-built apparatus shown in Figure 3.1. The remaining liquid was removed from the tubes and the green casts were dried inside the mould at room temperature for one day. After drying, the green casts were removed from the steel moulds and sintered horizontally in an oven. The green casts were sintered at temperatures ranging from 1000 to $1400^{\circ} \mathrm{C}$ for all three powder sizes. For the temperature range 1000 to $1200^{\circ} \mathrm{C}$ a Carbolite CWF1200 (Sheffield, England) oven was used and for the temperature range 1250 to $1400^{\circ} \mathrm{C}$ a Carbolite tube oven with a maximum temperature of $1600^{\circ} \mathrm{C}$ was used. The temperature regime for the sintering was as follows: 
- from room temperature to $400^{\circ} \mathrm{C}$ at a rate of $0.4^{\circ} \mathrm{C} / \mathrm{min}$

- constant heating for 15 minutes at $400^{\circ} \mathrm{C}$ to remove the APMA

- $400^{\circ} \mathrm{C}$ to maximum sintering temperature at a rate of $1^{\circ} \mathrm{C} / \mathrm{min}$

- constant sintering for 60 minutes at maximum sintering temperature

- maximum sintering temperature to room temperature at a rate of $1^{\circ} \mathrm{C} / \mathrm{min}$

The sintering temperatures employed for the temperature range experiments were: 1000,1050 , $1100,1150,1200,1250,1300,1350$ and $1400^{\circ} \mathrm{C}$. To calculate the repeatability of the production method, identical supports were produced using AKP-15 powder sintered at $1200^{\circ} \mathrm{C}$.

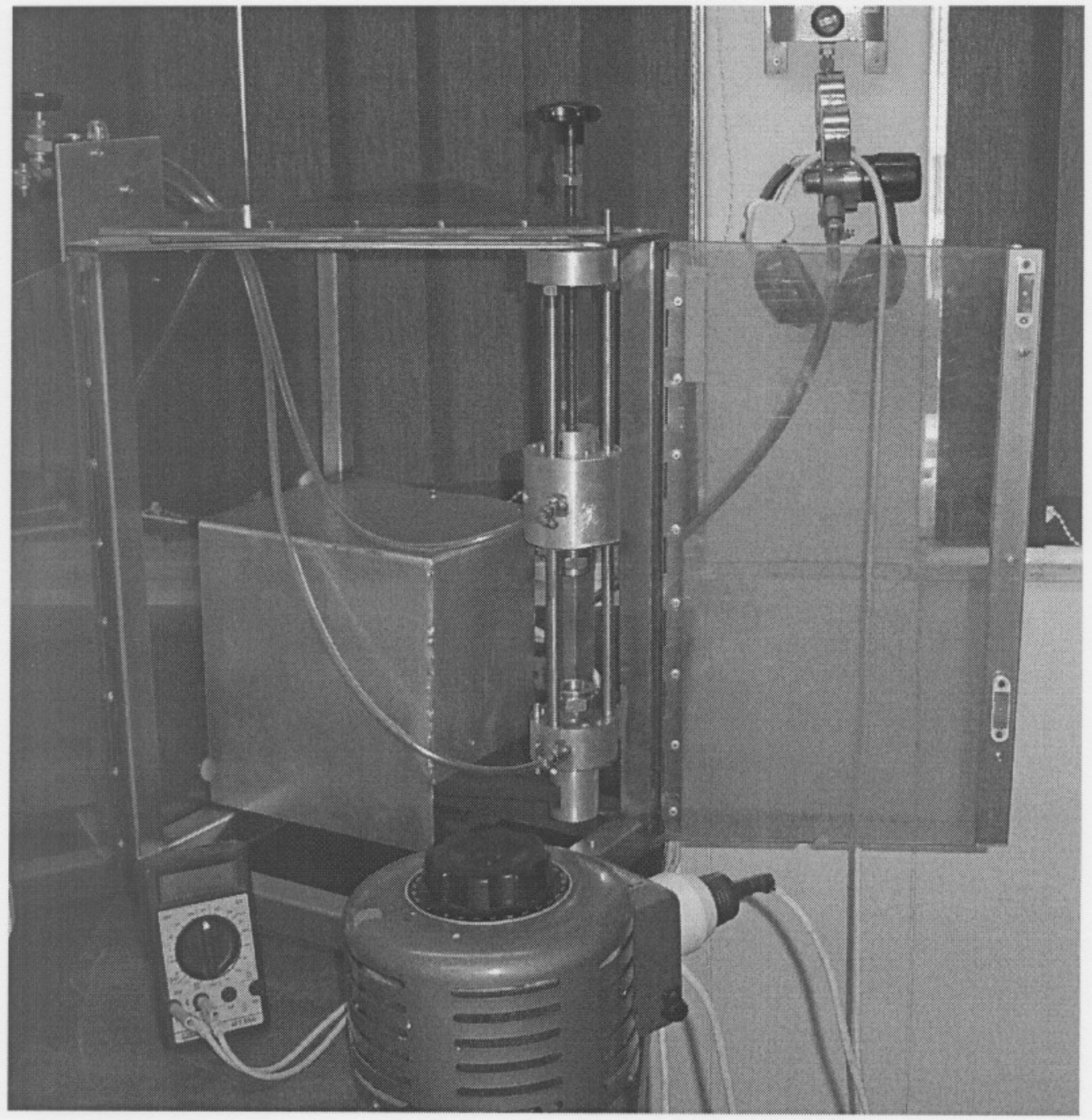

Figure 3.1 The custom-made centrifuge. 


\subsubsection{Influence of dispersant concentration and heating rate}

The influence of the amount of dispersant (APMA) on the properties of the ceramic support were also investigated. For this purpose, one series of supports were produced using the current amount of APMA (see section 3.2.1) and for the other series of supports the amount of free APMA in the suspension was halved. These supports were sintered at a temperature of $1100^{\circ} \mathrm{C}$ via the same temperature regime as indicated above. The influence of heating rate from room temperature to $400^{\circ} \mathrm{C}$ was also investigated. For this purpose one series of supports were heated at $0.4^{\circ} \mathrm{C} / \mathrm{min}$ to $400^{\circ} \mathrm{C}$ and another series of supports were heated at $1^{\circ} \mathrm{C} / \mathrm{min}$ to $400^{\circ} \mathrm{C}$. The remaining sintering regime was as described above in section 3.2.1 with the maximum temperature being $1100^{\circ} \mathrm{C}$. For this section AKP-30 powder was used.

\subsubsection{Characterization}

\subsubsection{Support dimensions and linear shrinkage}

The inner and outer diameters of the supports produced by the various powders, using varying amounts of dispersant and sintered at different temperatures, were measured with a Vernier caliper. To determine the linear shrinkage occurring during sintering, a distance of $49.80 \mathrm{~mm}$ was marked onto the green cast (unsintered) after drying. This distance was measured again after sintering and the percentage decrease was calculated.

\subsubsection{Mercury porosimetry}

Mercury porosimetry (Autopore III, Micrometrics) was used to determine the median pore radius, pore size distribution and the porosity of the various supports. For analysis, samples were dried at $120^{\circ} \mathrm{C}$ for at least 6 hours to remove excess moisture from pores and cavities in the ceramic support structure. For all analyses a penetrometer with part number 942-61707-00 and a total stem volume of $0.392 \mathrm{~cm}^{3}$ was used. The samples were loaded to obtain a used stem volume of between 60 and $90 \%$ under high pressure intrusion. 
The density of the support structure was calculated by obtaining the bulk density at 1.02 psia as a percentage of the apparent (skeletal) density. The porosity is then acquired by subtracting the density from $100 \%$. The Washburn equation was used to determine the median pore diameter of the support structure.

\subsubsection{Water permeability}

The water permeability of the ceramic membranes was tested using a set-up consisting of a $\mathrm{N}_{2}$ gas supply, a water storage vessel and a permeation cell. The experimental set-up is shown in Figure 3.2. The nitrogen gas provided the driving force (pressure) for the pure water feed to permeate through the support that has been sealed in the membrane module at the top and bottom by two O-rings as shown in Figure 3.3. The module was used in the dead-end mode i.e. one inlet and one outlet remained closed. The volumetric flow rate was calculated at 5 different pressures (2-10 bars). A mass balance was used to determine the flow through the membrane.

\subsubsection{SEM}

The microstructure of the ceramic membrane was studied by means of SEM. A SEM of the type Philips XL30 was used to obtain images of the inside as well as cross sections of the support. 


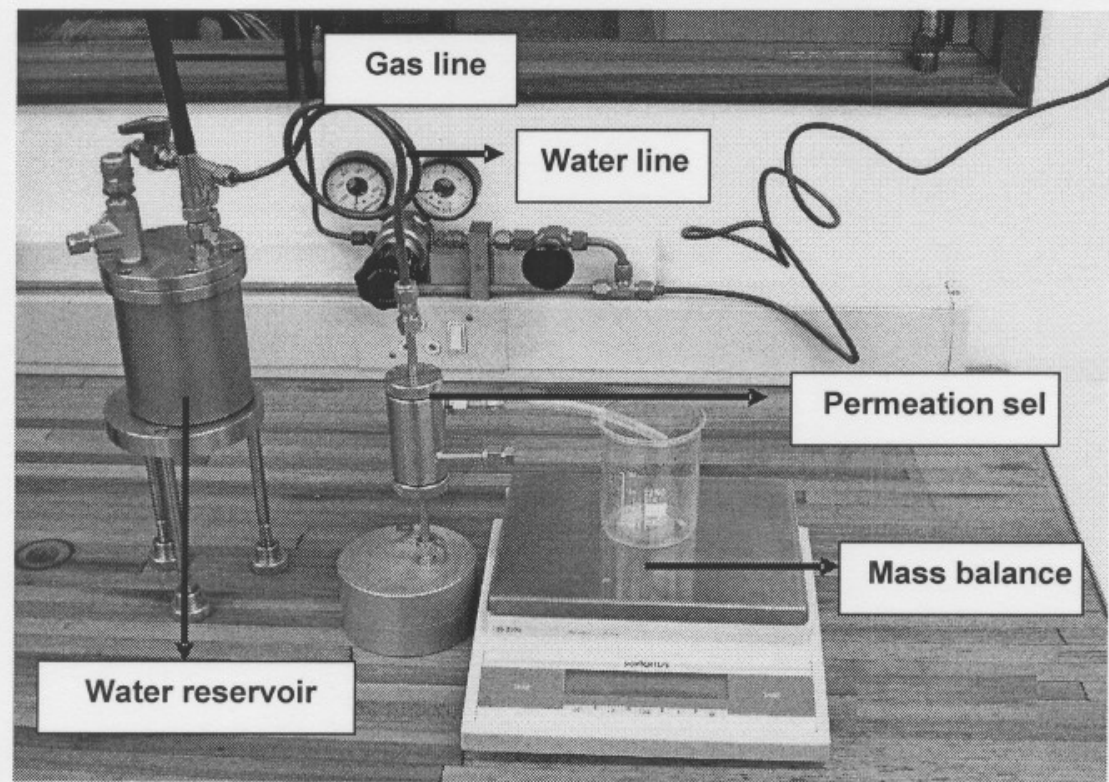

(a)

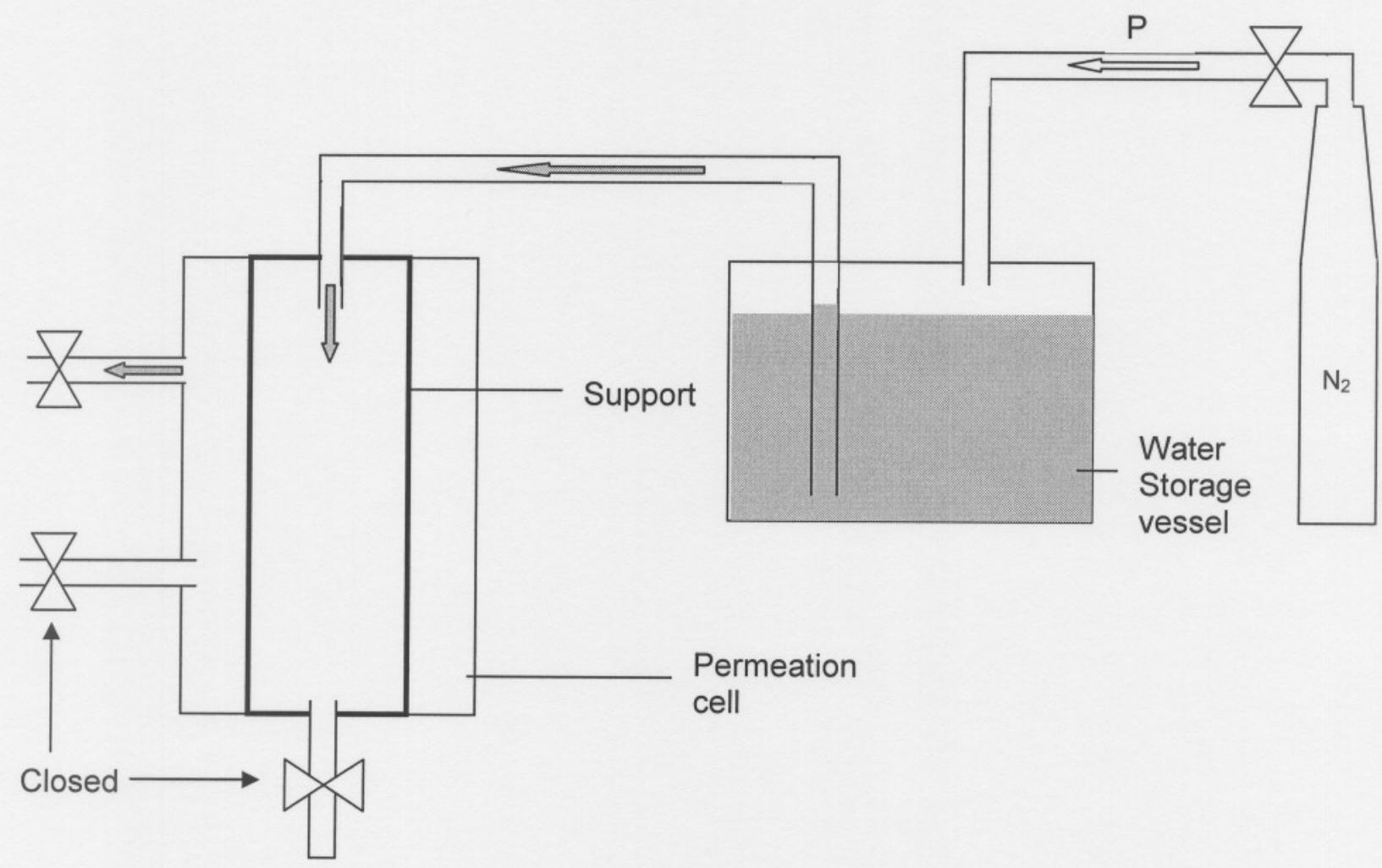

(b)

Figure 3.2 A photograph (a) and a schematic representation (b) of the water permeability setup. 


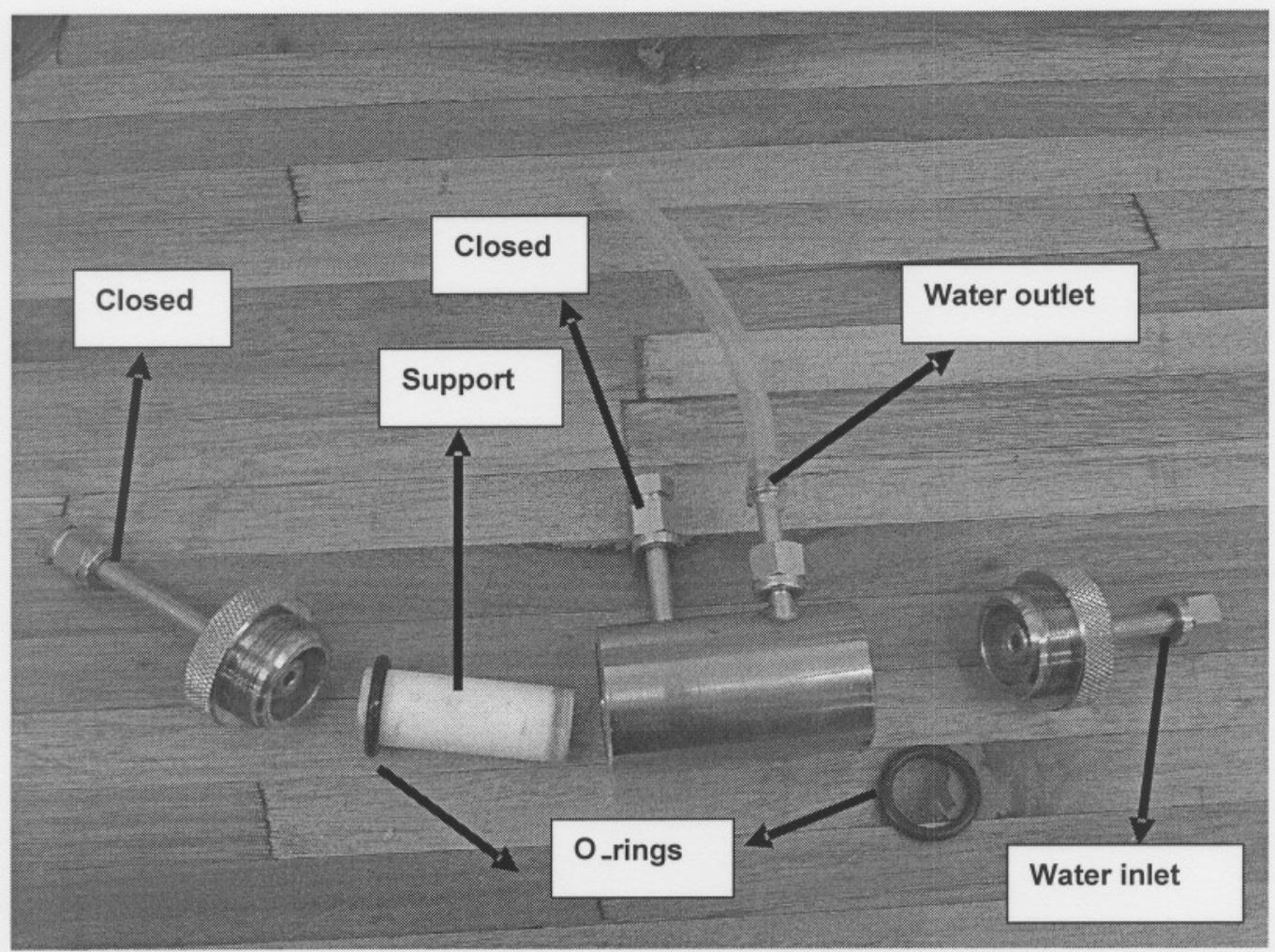

Figure 3.3 The membrane and the membrane module for the water permeability studies.

\subsubsection{Strength test}

To evaluate the strength of the supports a novel apparatus was developed to exert pressure on the inside of the tubular structure. The device consists of two metal pieces as shown in Figure 3.4, which were placed inside the ceramic support structure opposite from each other. By pressing the cone shown in Figure $3.4 \mathrm{~b}$ in between these two pieces along the grooves, the two pieces were pushed in opposite directions resulting in a force being applied on the inside wall of the support structure. The cone was forced into the ceramic support using a Tensometer Type W (A.R.Adams Trading) which displayed the force applied in Newton. The force was increased until breakage or rupturing occurred. 


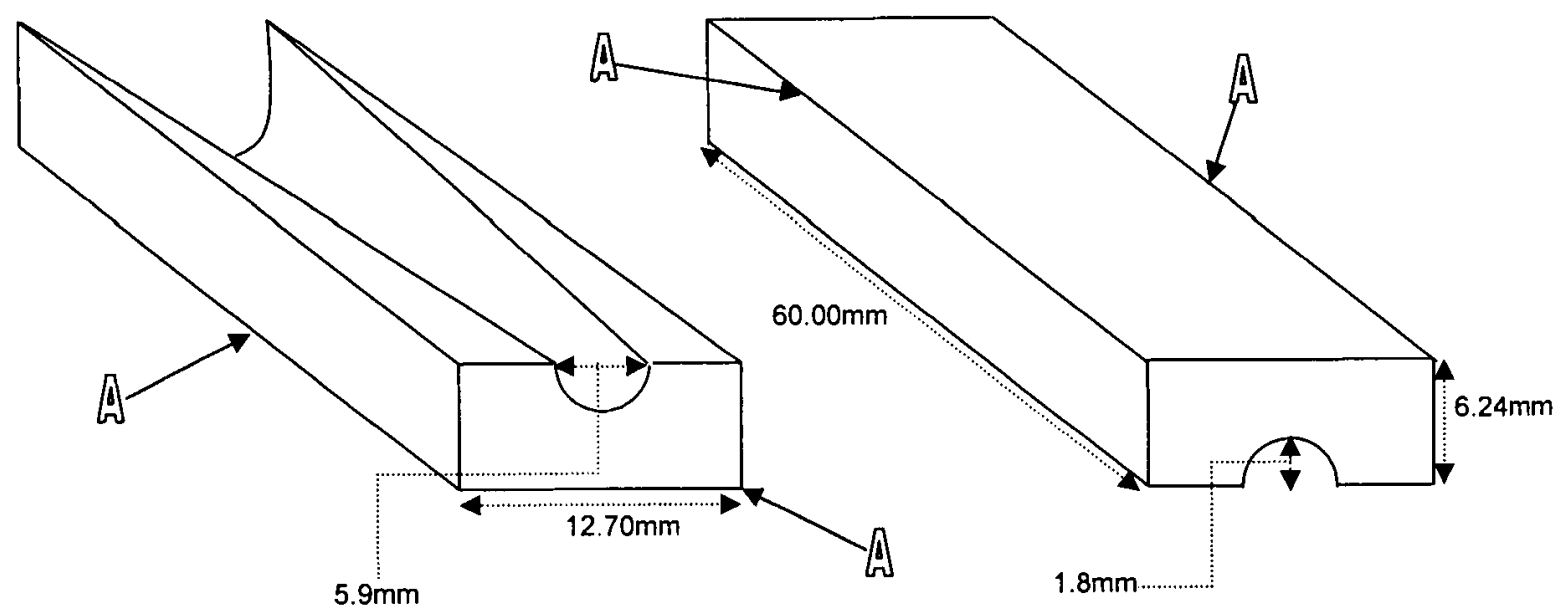

(a)

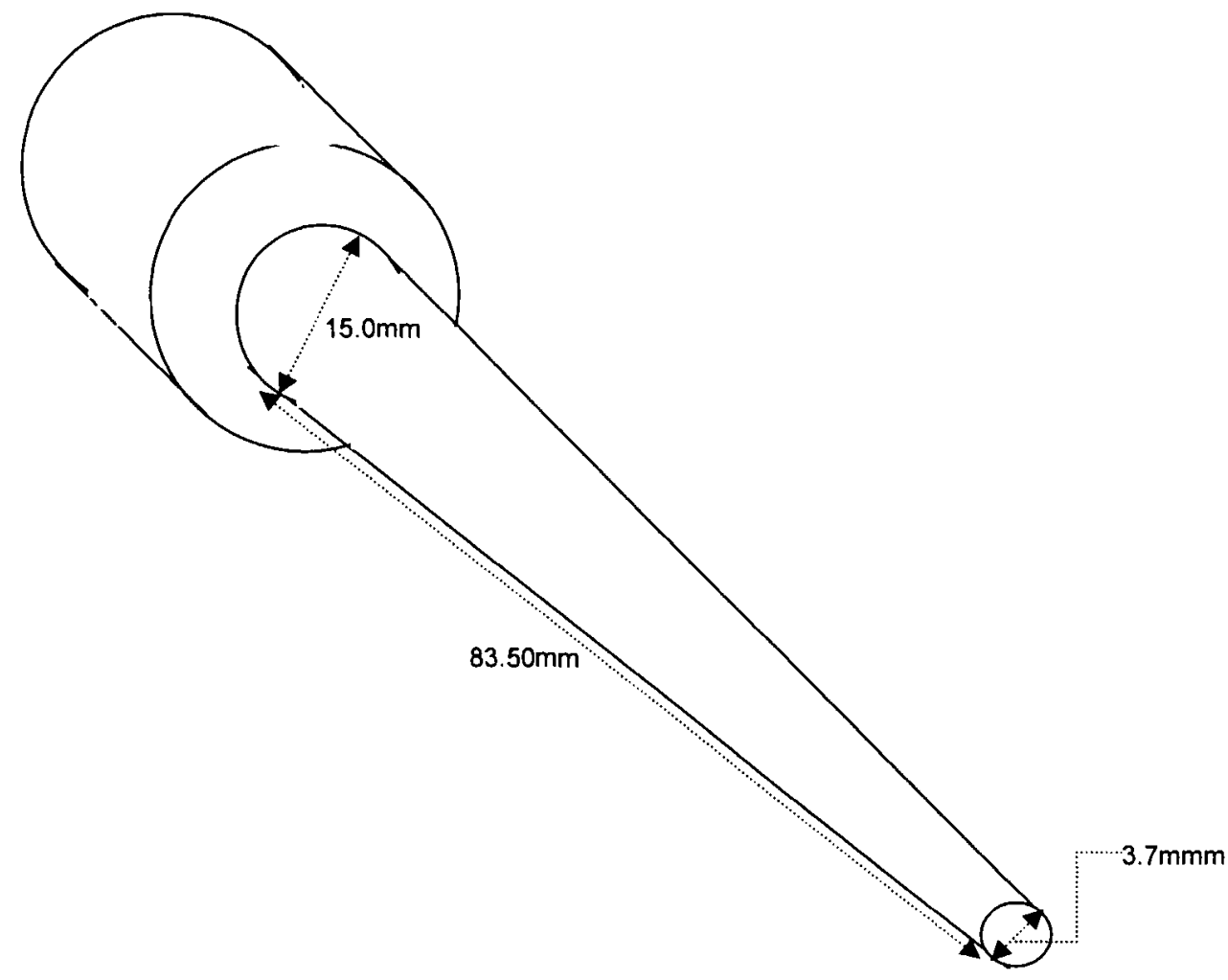

(b)

Figure 3.4 The strength testing apparatus consisting of two metal pieces with conical cavities (a) and the cone (b). 
Due to the specific design of the metal pieces only the sections " $A$ " were in contact with the support structure. The force applied can be converted to pressure using the following equation:

$$
\text { Pressure }(\mathrm{Pa})=\frac{\text { Force }(\text { Newton })}{\operatorname{Area}\left(\mathrm{m}^{2}\right)}
$$

The contact area " $A$ " was calculated according to equation (3.2),

$$
\text { Area }=L \times w
$$

where $w$ is the width $(\mathrm{mm})$, and $L$ the length of the contact section $(\mathrm{mm})$. Since the metal pieces are longer than the membrane supports, $L$ equals the length of the support which was $58 \mathrm{~mm}$. The width of the contact section was approximately $0.341 \mathrm{~mm}$ according to SEM. Since there were two metal pieces (Figure 3.3a) and each metal piece had two sections ("A") exerting a force on the support, the total area was equal to $4 \times \mathrm{L} \times$ w which equals $7.91 \times 10^{-5} \mathrm{~m}^{2}$.

\subsection{Results and Discussion}

\subsubsection{Repeatability}

The variation in water permeability and tensile strength for the three AKP-15 supports sintered at $1200^{\circ} \mathrm{C}$ were $0.6 \mathrm{ml} \mathrm{s}^{-1} \pm 1.6 \%$ or $41.1 \pm 0.7 \mathrm{l} . \mathrm{m}^{-2} \mathrm{~h}^{-1}$ bar. $^{-1}$ for the permeation results and $100 \mathrm{~N} \pm$ $7.1 \%$ or $403 \pm 28.5 \mathrm{MPa}$ for the tensile strength test. Since this variation is the sum of the variation of the synthesis method and the characterization method it is clear that membrane supports made by centrifugal casting are highly reproducible considering a combined manufacturing and permeation error of less than $2 \%$.

Assuming a $2 \%$ error in the manufacturing process would mean that the error of the novel tensile strength test is not more than $5 \%$ which is acceptable for all practical purposes.

The presented deviation for the mercury intrusion porosimetry (pore size and porosity) results are not presented because results differ for every analysis since the pore size and porosity depend on the intrusion volume and stem volume of each analysis. In general there was a $5 \%$ error for the porosity measurements and $3 \mathrm{~nm}$ radii deviation observed for each analysis.

The amount of linear shrinkage (\%) obtained for the three AKP-15 supports sintered at $1200^{\circ} \mathrm{C}$ was $2.77 \pm 0.34 \%$, which was a $12 \%$ standard deviation. 


\subsubsection{Effect of sintering temperature and powder size}

\subsubsection{Support dimensions and linear shrinkage}

According to Table 3.1 , it was confirmed that the sintering temperature had an effect on the dimensions of the different supports. Only the outer diameter is presented since the changes in inner and outer diameter as a function of temperature remained the same. The dimensions of the supports were influenced by the temperature at which they were sintered as well as the particle size of the powder used. The outer diameter of the AKP-15 supports decreased from $21.20 \mathrm{~mm}$ $\left(1050^{\circ} \mathrm{C}\right)$ to $19.90 \mathrm{~mm}\left(1300^{\circ} \mathrm{C}\right)$ which was a decrease of $6.13 \%$, while the outer diameter of AKP30 and AKP-50 supports decreased by $11.81 \%$ and $12.59 \%$ respectively. It is thus clear that there was a more profound decrease in outer diameter with a decrease in particle size. This observation correlates with that of Steenkamp., ${ }^{10}$ who found that smaller particles packed equivalently will produce an equivalent shrinkage in a shorter time because of more contact points per unit volume and thus a larger driving force for shrinkage. A similar observation was made when the linear shrinkage was evaluated as a function of sintering temperature. From Figure 3.5 it can be seen that the linear shrinkage rate for the AKP-50 supports were higher than that of the AKP-30 supports, whilst the AKP-15 supports had the lowest rate of shrinkage due to the faster shrinkage of the smaller particles. ${ }^{10}$

The difference in linear shrinkage between the supports sintered at $1050^{\circ} \mathrm{C}$ and $1300^{\circ} \mathrm{C}$ for the AKP-15 powder was $5.17 \%$, while it was $10.62 \%$ and $13.59 \%$ for the AKP-30 and AKP-50 powders, respectively. For the AKP-15 and AKP-30 powders the linear shrinkage was thus less than the shrinkage of the diameter, while the opposite was observed for the AKP-50 supports. This slight difference in the axial (linear) and radial (diameter) shrinkage is due to the difference in the direction of shrinkage occurring during sintering. This is called shrinkage anisotropy and occurs because shrinkage starts at the most-"shrinkable" sites in the specimen which could be due to the difference in the number of nearest-neighbor contact points. ${ }^{11}$ According to Shui et al., ${ }^{12}$ shrinkage differences between linear and diametrical directions could be due to differences in the densities of packing in these directions. This would explain the results obtained for the AKP-15 and AKP-30 powder where a definite density gradient exists in the support due to centrifugal casting. For the AKP-50 supports, however, the opposite was observed which implies a decrease in the density gradient which could be due to a decrease in the particle size distribution of the AKP50 powder. 
Shui et al. ${ }^{12}$ also noted that the orientation of the particles could be important, since the shrinkage is usually larger in the direction perpendicular to alignment, but in compacts manufactured from spherical particles there is no orientation or anisotropic sintering shrinkage. Thus it should be concluded that the difference in axial and radial shrinkage is due to differences in the density gradient of the support structures especially for AKP-15.

In Figure 3.5 it is interesting to note that the change in linear shrinkage might be a power function. When a graph of outer diameter (Table 3.1) of the supports as a function of temperature is plotted (graph not shown) it confirms the trend observed in Figure 3.5. Zeng et al. ${ }^{2}$ proposed an equation to express a relationship between linear shrinkage and time. They proposed equation (3.3).

$\Delta L / L_{0}=1-\left(\rho / \rho_{g}\right)^{1 / 3}$

where $\Delta \mathrm{L} / \mathrm{L}_{0}$ is the linear shrinkage rate, $\rho$ is the density of the specimen and $\rho_{\mathrm{g}}$ the green density. This equation is a power function, confirming the observation made in this study.

Table 3.1 Outer diameters of AKP-15, AKP-30 and AKP-50 tubular supports sintered at various temperatures

\begin{tabular}{cccc}
\hline $\begin{array}{c}\text { Sintering } \\
\text { temperature }\left({ }^{\circ} \mathrm{C}\right)\end{array}$ & AKP-15 & AKP-30 & AKP-50 \\
\hline 1050 & 21.20 & 21.00 & 20.65 \\
1100 & 21.05 & 20.78 & 20.33 \\
1150 & 20.83 & 20.33 & 19.84 \\
1200 & 20.65 & 19.97 & 19.30 \\
1250 & 20.30 & 19.48 & 18.58 \\
1300 & 19.90 & 18.52 & 18.05 \\
1350 & 19.25 & - & - \\
1400 & 18.74 & - & - \\
\hline
\end{tabular}




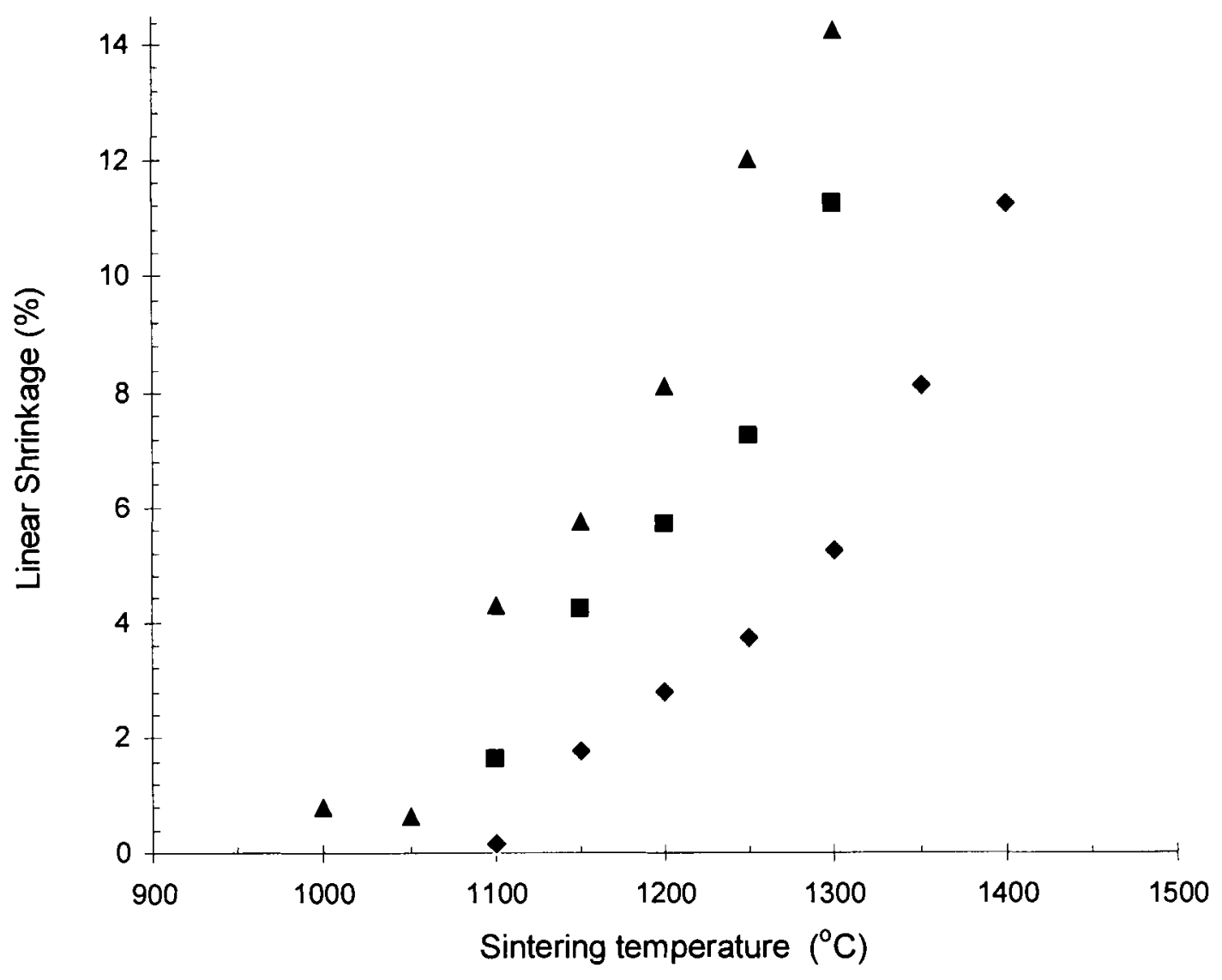

Figure 3.5 Linear shrinkage as a function of sintering temperature for $A K P-50(\Delta), A K P-30(\square)$ and $A K P-15(\bullet)$ powder.

\subsubsection{Mercury porosimetry}

The porosity was affected by the sizes of the particles as well as the temperature at which the supports were sintered as can be seen from Figure 3.6. For all powders there was a decrease in porosity with temperature increase and particle size decrease. There was a faster increase in density for the smaller particles, as the supports produced with the AKP-50 $(0.25 \mu \mathrm{m})$ powder had the lowest porosity whilst the AKP-15 $(0.62 \mu \mathrm{m})$ had the highest porosity at elevated sintering temperatures. This is because the smaller particles have a denser particle packing within the support structure. The decrease in porosity with sintering temperature increase is due to pore shrinkage or densification of particles occurring at elevated temperatures, especially when grain boundary and lattice diffusion occurs. The porosity decrease is more rapid for the smallest particle sizes as was observed by Steenkamp. ${ }^{10}$ 


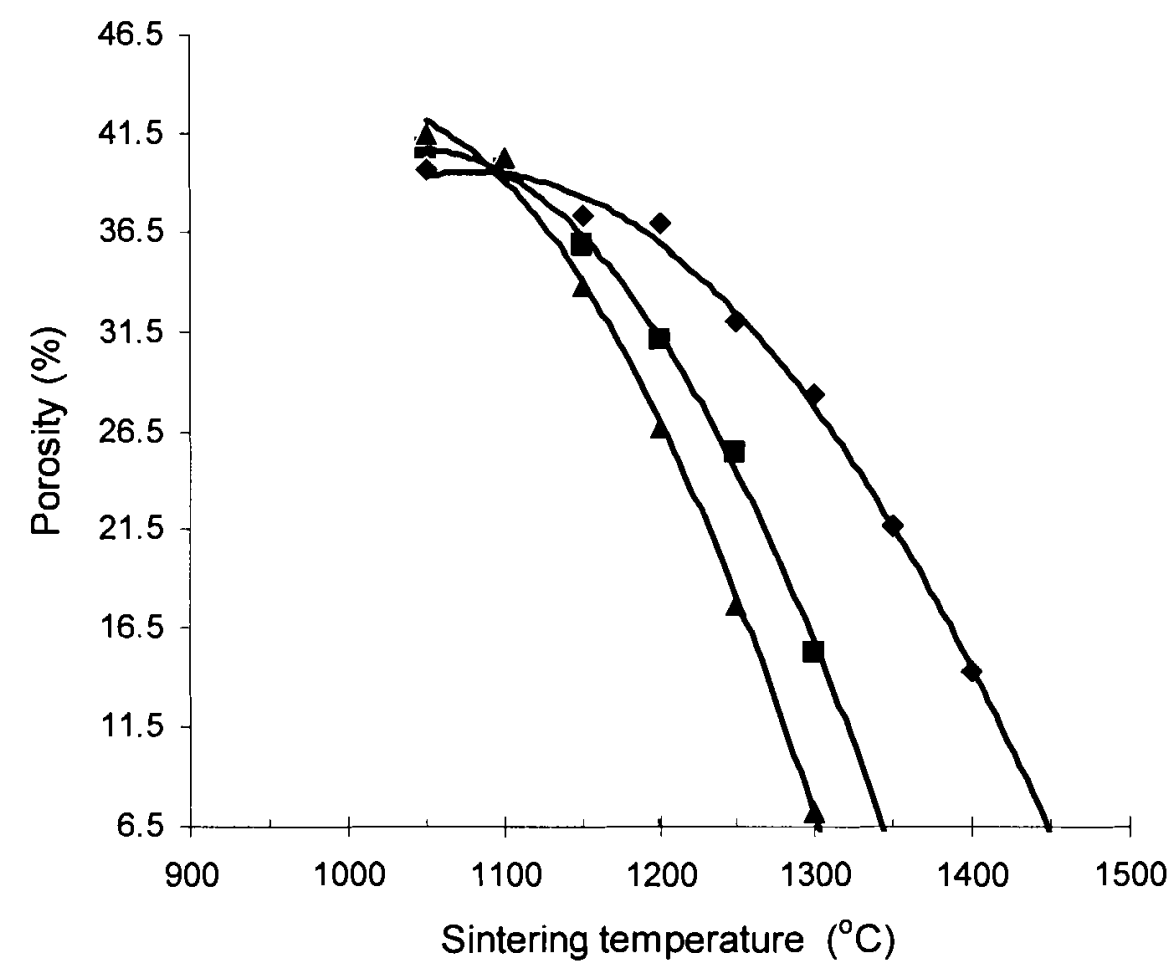

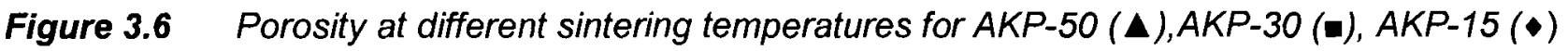
powder.

Kingery et al. ${ }^{13}$ stated that, according to classical sintering theory, there are two competing processes in conventional sintering, densification and grain growth, and both are driven by capillary forces which are proportional to the grain size. Thus, the smaller the initial powder size, the larger the densification and grain growth rates during sintering. ${ }^{13}$ Herring's scaling law also states that fine particles promote faster sintering. ${ }^{14}$ This more rapid pore shrinkage or particle densification of the smaller particles also explains the more rapid structural shrinkage observed for the various supports. The decrease in porosity (or increased densification) with increased sintering temperature confirms results obtained by Kim et $a l^{15}$ and Steenkamp et al. ${ }^{16}$ The model calculations of Kang et al., ${ }^{17}$ who determined the densification curves of alumina powder compacts with $0.8 \mu \mathrm{m}$ and $4 \mu \mathrm{m}$ particles, also confirms this trend. Although Darcovich et al. ${ }^{18}$ obtained porosity data that gave maximum porosity at higher temperatures, for some samples they also observed that the porosity decreased slightly as the sintering temperature increased, suggesting densification through shrinkage and fusion. In their study, Nijmeijer et al. ${ }^{27}$ produced centrifugal casted (at 20000rpm) AKP-15 and AKP-30 supports sintered at $1150^{\circ} \mathrm{C}$ which resulted in structures with porosities of $34.8 \%$ and $34.5 \%$ respectively, while the support porosities in this study were $37.21 \%$ and $35.75 \%$ for the AKP-15 and AKP-30 respectively, indicating an overall 
improvement in terms of porosity. This improvement is mainly due to the optimization of the suspension properties and the sintering conditions.

While all membranes in this study were sintered for only 1 hour, the duration of sintering influences the porosity of the final product. This observation was made in a study by Biesheuvel et al. ${ }^{23}$ who manufactured tubular ceramic membranes by a vacuum casting process. They found that the longer the sintering time at the maximum temperature, the lower the porosity. The porosity decreased from $32 \%$ at zero hours sintering time to $28 \%$ at 5 hours and to $27 \%$ at 10 hours, respectively.

The pore radius was affected both by the particle size and sintering temperature as can be seen from Figure 3.7. The influence of sintering temperature on pore radius decreased with decreasing particle size. While there is a definite decrease in pore size for the AKP-15 from nearly $100 \mathrm{~nm}$ to just under $70 \mathrm{~nm}$, the pore size for the AKP-50 powder remained nearly constant at approximately $35 \mathrm{~nm}$. The constant pore size of the AKP-50 supports with increased sintering temperature could be explained as followed: The surface area occupied by the internal pores located at grain junctions depends on the number of the surrounding grains. If a pore is surrounded by more than 6 grains, the pore would thermodynamically grow during sintering; if the pore is surrounded by six grains the pore size would be stable, while the pores would shrink if they were surrounded by fewer than six grains. ${ }^{19}$ The amount of grains surrounding a specific pore also influences the density explaining the difference observed in the radial and axial shrinkage of the AKP-15 and AKP-30 versus the AKP-50 supports shown in Section 3.3.2.1. According to this theory it would mean that, during centrifugation, the AKP-50 powder is arranged in such a manner that each pore is surrounded by 6 or more grains leading to pore growth during sintering. For the AKP-30 supports there were fewer than 6 grains surrounding each pore and even fewer neighboring grains for the AKP-15, resulting in a pore size decrease during sintering. 


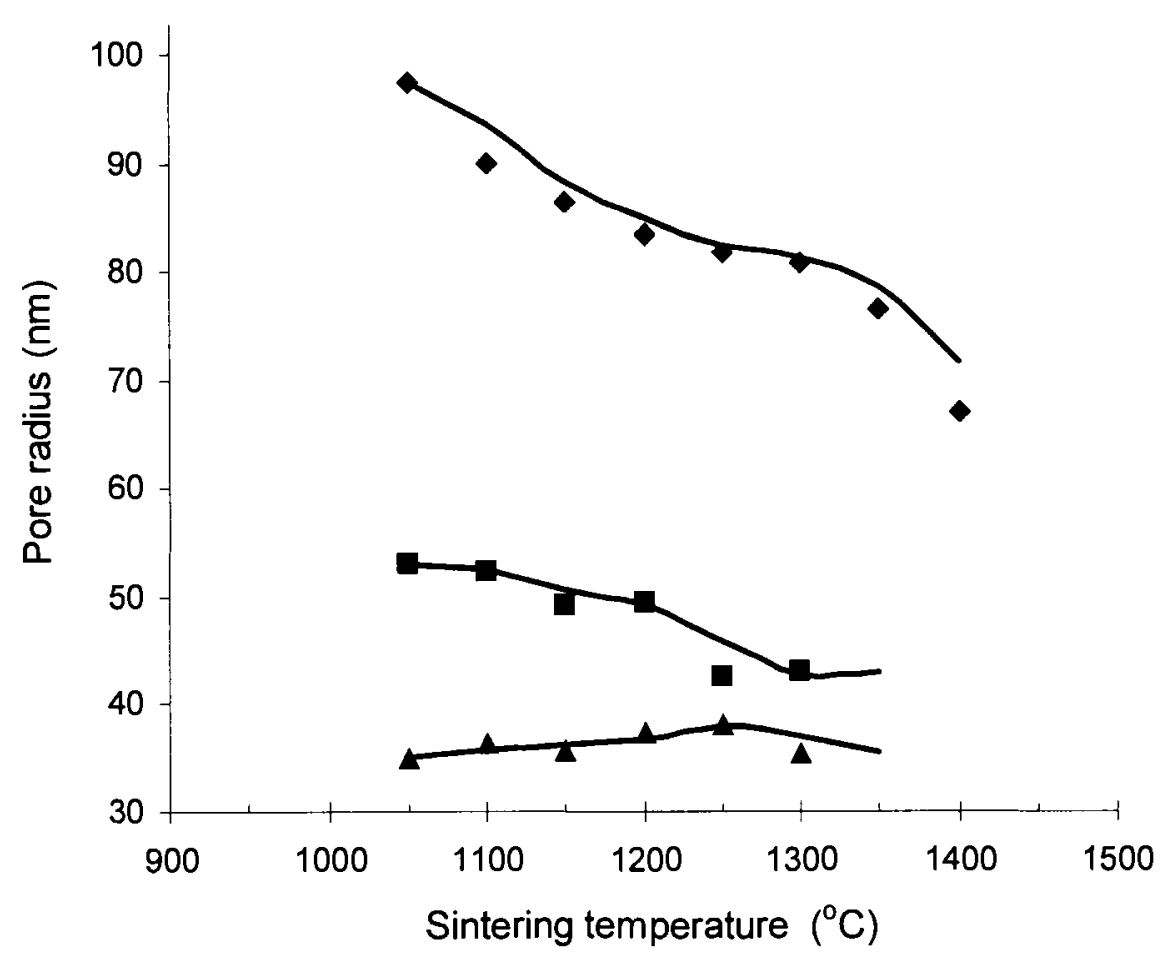

Figure 3.7 Pore radius at different sintering temperatures for AKP-50 (A),

AKP-30 (ロ), AKP-15(•) powder.

Another approach to explain the constant pore size with increased sintering temperature was presented by Wang et al. ${ }^{20}$ who produced membrane supports by slip casting using $0.5 \mu \mathrm{m}$ and $2.8 \mu \mathrm{m}$ powders. They suggest that, according to the topological decay model of sintering, the pore radius remains constant during sintering, but that the total length and number of channels (pores) decrease resulting in a decrease in porosity.

In another study, Kim et al. ${ }^{15}$ made alumina tubes by centrifugal casting at $2500 \mathrm{rpm}$ using $0.5 \mu \mathrm{m}$ and $0.8 \mu \mathrm{m}$ a-alumina particles. For the supports consisting of $0.5 \mu \mathrm{m}$ particles, the median pore radius remained constant with increasing sintering temperature, while the median pore radius for the $0.8 \mu \mathrm{m}$ supports were larger than for the $0.5 \mu \mathrm{m}$ support. The porosities for both particle sizes were identical. According to their findings the porosity depends more on the sintering temperature, while the pore size is more dependent on the size of the starting powder used for the membrane manufacture. The particle size influences the particle packing and thus the pore radius evolution during sintering. These findings correspond to our results. However, using the $0.5 \mu \mathrm{m}$ particles they obtained a constant pore size as a function of temperature, while in our study a decrease in pore size with increasing sintering temperature was observed both for the smaller AKP-30 
$(0.31 \mu \mathrm{m})$ and the larger AKP-15 $(0.61 \mu \mathrm{m})$ powder. Only for the AKP-50 $(0.25 \mu \mathrm{m})$ the independence of pore radius as a function of sintering temperature was illustrated. This difference could be due to the fact that the supports they produced were manufactured at a much lower centrifugal speed $(2500 \mathrm{rpm})$, resulting in a less dense particle packing i.e. larger pores surrounded by 6 or more grains, resulting in a more stable pore size with increased sintering temperature.

In Figure 3.8 the pore volume as a function of pore diameter for the AKP-15 supports sintered at various temperatures is illustrated. There was an overall decrease in median pore diameter and pore volume with increased sintering temperature. The pore size distribution was narrow and remained nearly constant as was observed by Kim et al. ${ }^{15} \mathrm{~A}$ similar trend was obtained for the AKP-30 support. The only difference was that the AKP-30 supports had a smaller median pore diameter compared to the AKP-15 supports. The decrease in pore volume indicates the decrease in porosity as was seen earlier in Figure 3.5, while the decrease in mean pore diameter is the decrease in pore radius for the AKP-15 and AKP-30 supports discussed previously.

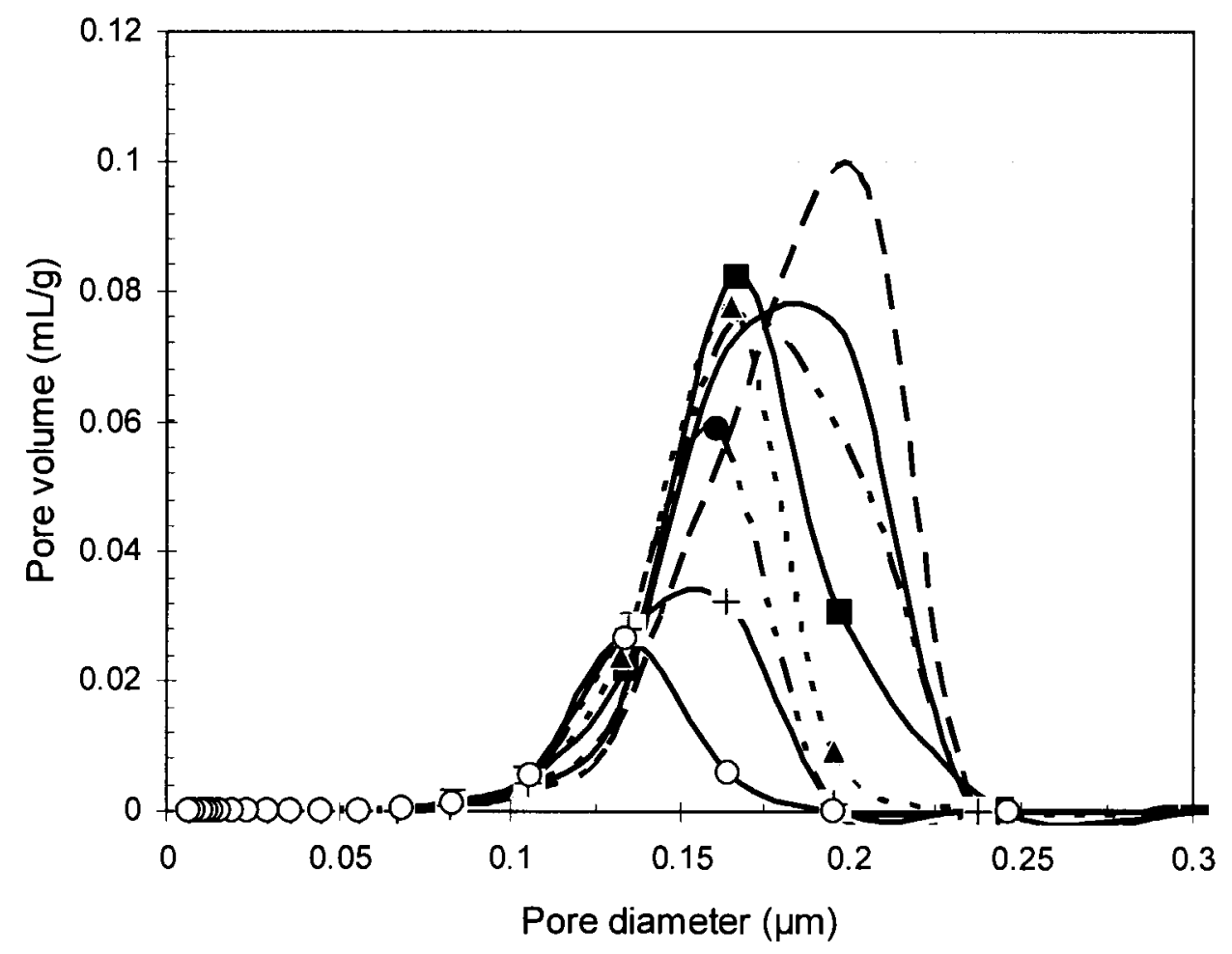

Figure 3.8 Pore volume as a function of pore diameter for the AKP-15 supports sintered at various temperatures.
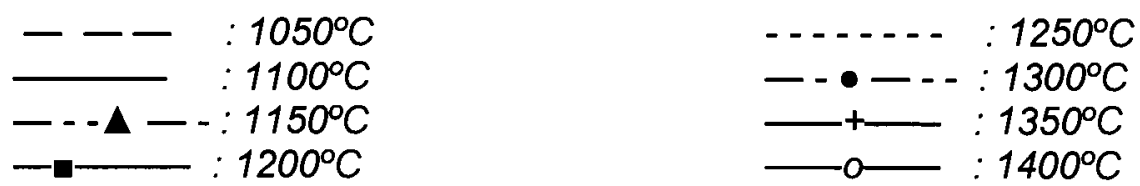
In Figure 3.9, a graph of pore volume against pore diameter for the AKP-50 powder is presented. The mean pore diameter of the AKP-50 supports decreased when compared to the AKP-15 supports, as shown in Figure 3.8. According to Figure 3.9, the pore size distribution for the AKP50 powder was narrow and stayed mainly constant while the pore volume decreased with increasing sintering temperature. The pore diameter remained constant or slightly increased with increasing sintering temperature. Figure 3.8 clearly illustrates the decrease in pore size and porosity for the AKP-15 and AKP-30 (which are similar) supports with increasing sintering temperature. The constant pore size and decrease in porosity for the AKP-50 powder with increased sintering temperature, as discussed earlier, is shown in Figure 3.9.

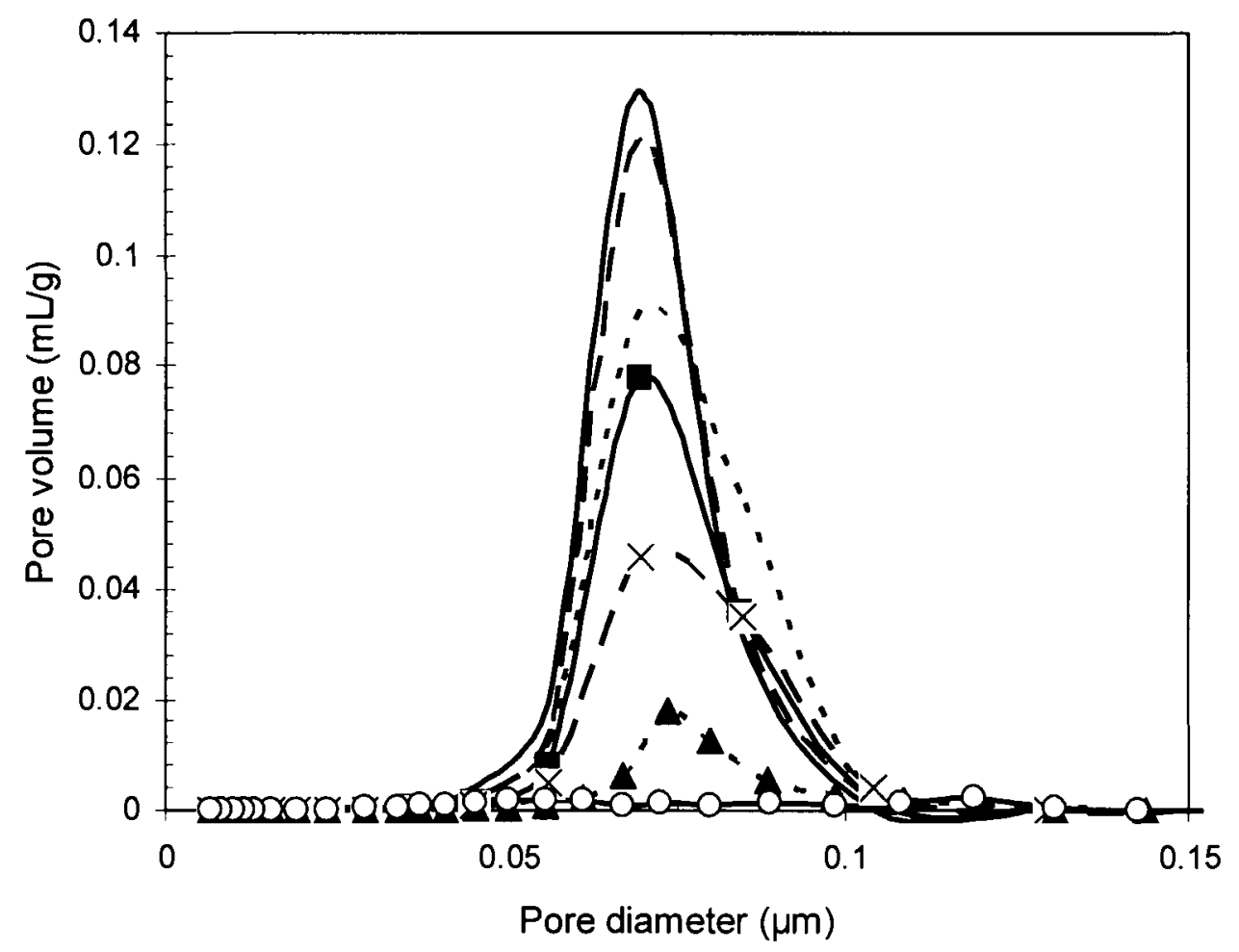

Figure 3.9 Pore volume as a function of pore diameter for the AKP-50 supports at various temperatures.
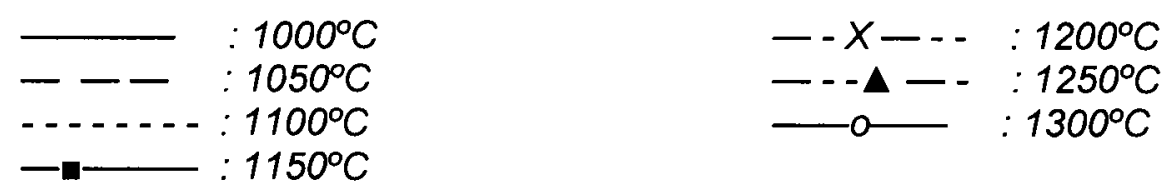

Figure 3.10 illustrates the effect of particle size at a constant sintering temperature of $1200^{\circ} \mathrm{C}$ on the pore volume and pore diameter of the tubular structures. The mean pore diameter as well as the pore volume (porosity) decreased with decreasing particle size of the starting powder. While 
the study done by Kim et al. ${ }^{15}$ has shown that the porosity depends more on sintering temperature than the size of the starting powder used for the membrane manufacture, we did find a significant change in porosity although the sintering temperature remained constant. It has clearly been shown in this study that the particle size does have an influence on the porosity of the support structure, even though it may be less profound than the influence of sintering temperature.

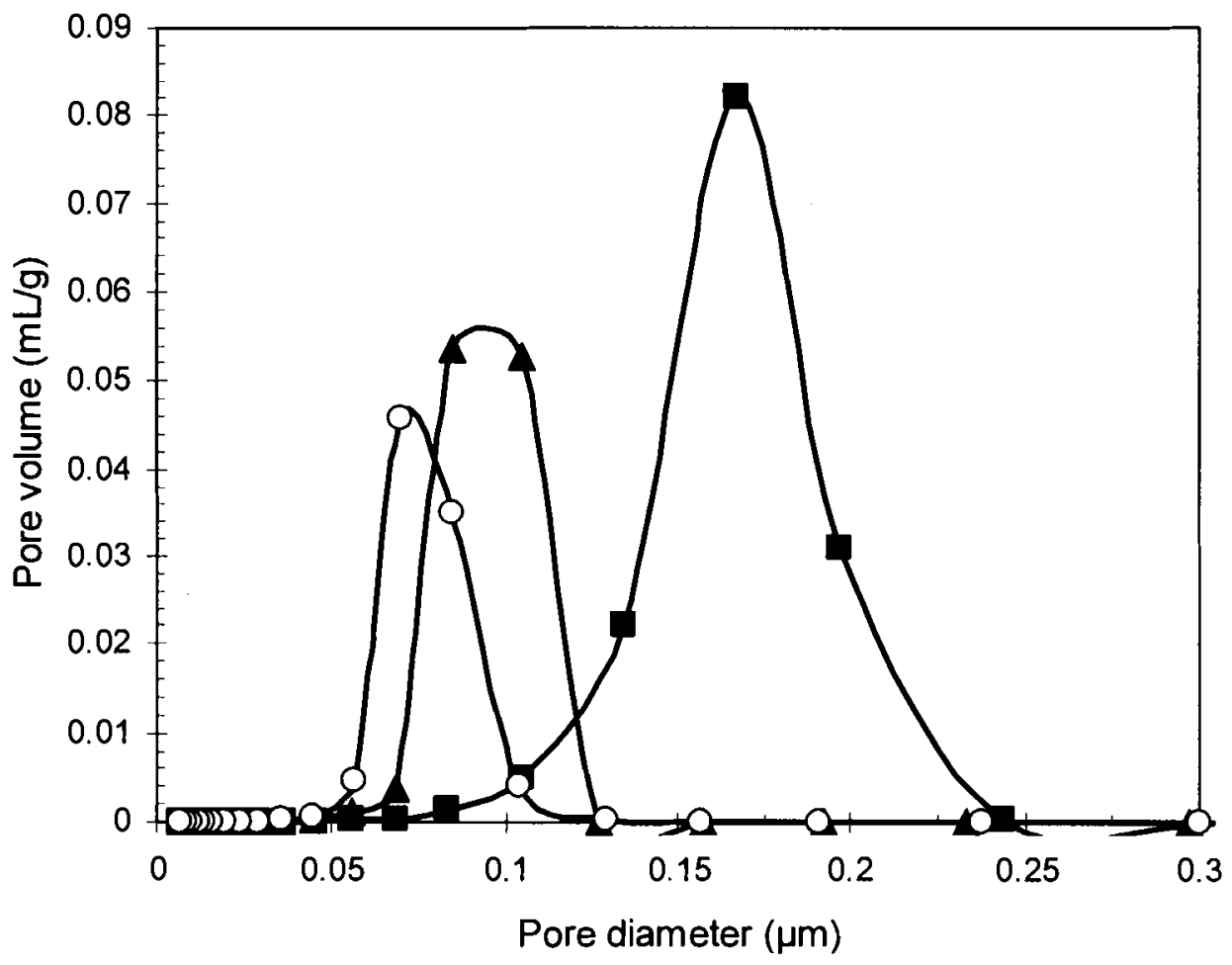

Figure 3.10 Pore volume as a function of pore diameter for the alumina supports sintered at $1200^{\circ} \mathrm{C}$.

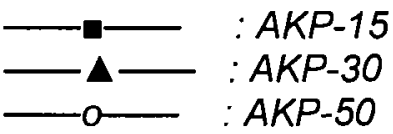

In an attempt to quantify the effect of sintering temperature on the pore size distribution, the standard deviation was calculated from the mercury intrusion data. To calculate the standard deviation, equation (3.4) was used, where $S$ is the standard deviation, $w$ is the pore volume at a specific pore diameter $(y)$ and $n$ the total amount of data points used in the calculation. The standard deviation indicates the deviation from the weighed average of the distribution. In short it indicates the width of a specific distribution. 
$S=\sqrt{\frac{\sum w y^{2}-\left(\sum w y\right)^{2}}{n-1}}$

Using the above mentioned method, a decrease (narrowing) in the standard deviation of the pore size distribution with an increasing sintering temperature was obtained for the AKP-15, AKP-30 and the AKP-50 supports. According to Figure 3.11, AKP-15 had the widest pore size distribution, while the pore size distributions for AKP-30 and AKP-50 were similar. Biesheuvel et al. ${ }^{21}$ illustrated in their study that the AKP-15 powder had a wider particle size distribution than the AKP-30 powder, with the AKP-50 powder having the narrowest distribution. It is known that the particle size distribution (PSD) has an influence on the pore size distribution, as a narrow PSD results in a narrow pore size distribution, while a wide PSD would result in a wider pore size distribution. ${ }^{22}$ The wider pore size distribution obtained for the AKP-15 powder confirms the statement by Das et al. ${ }^{22}$ that a wider PSD would result in a structure with a wider pore size distribution. However, it would then have been expected that the AKP-50 pore size distribution would be narrower than the AKP30 pore size distribution. Although the standard deviations were slightly smaller, in general the difference was not statistically significant. This behavior could be attributed to the difference in particle arrangement as discussed in Section 3.3.2.1. Due to the fact that the pore radius remained nearly constant for the AKP-50 supports with increasing sintering temperature, the pore size distribution remained wider than would be expected for an AKP-50 support with a similar particle packing as the AKP-30 and AKP-15 supports. The narrowing of the pore size distribution with increased sintering temperature was attributed to the shrinkage occurring during sintering. This was observed for all the support structures. 


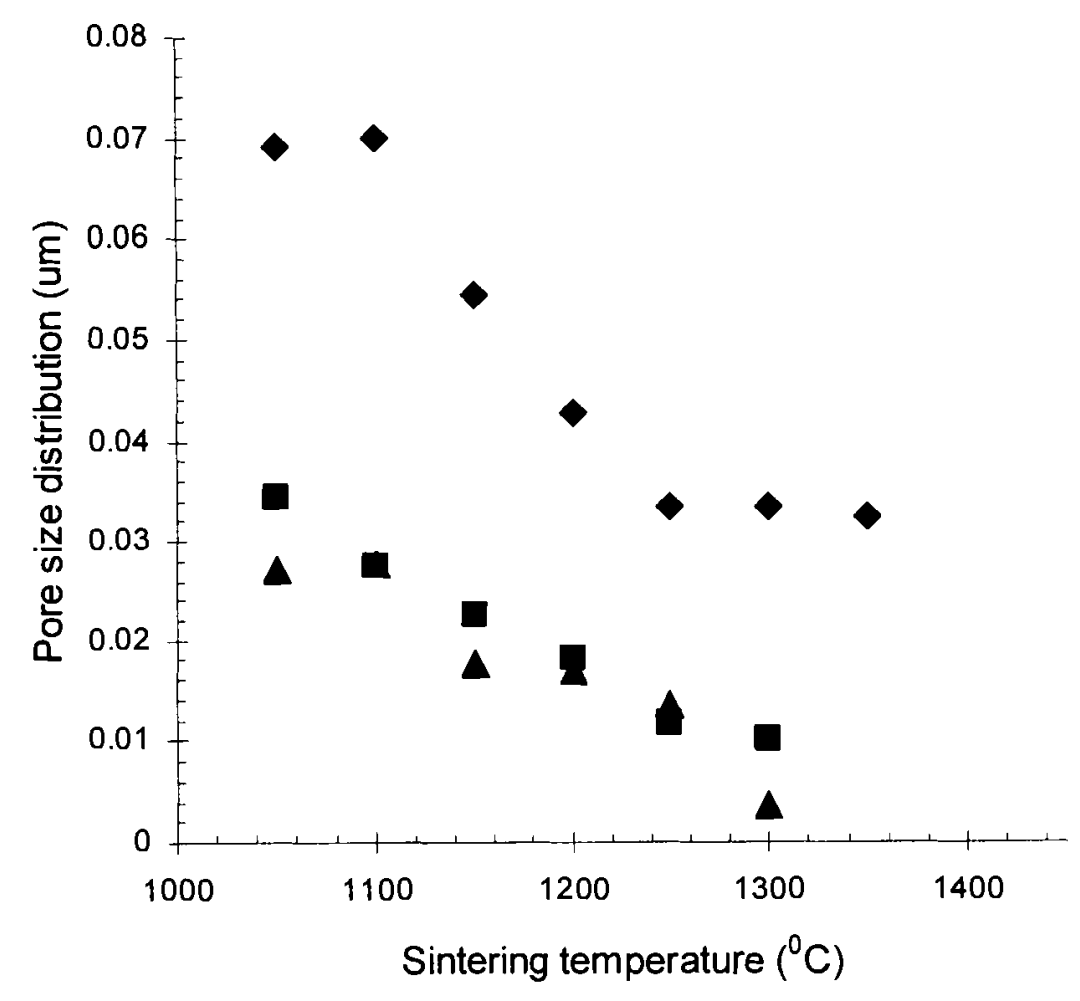

Figure 3.11 Pore size distribution of the pore size distribution of the various supports as a function of sintering temperature.
- :AKP-15
- $: A K P-30$
$\Delta: A K P-50$

\subsubsection{Water permeability}

The water permeability though a porous structure is influenced by a number of variables according to the Hagen Poiseuille equation

$$
J=\frac{\varepsilon}{\tau} \frac{r^{2}}{8 \eta} \frac{\Delta P}{\Delta x}
$$

where $J$ is the water flux through the membrane $\left(1 / m^{2} . h\right), r$ is the pore radius $(m), \varepsilon$ is the porosity ( ), $\Delta P$ the pressure difference across the membrane $(\mathrm{Pa}), \Delta x$ the membrane thickness $(\mathrm{m}), \tau$ the tortuosity $(-)$ and $\eta$ the viscosity of water $(\mathrm{Pa} . \mathrm{s})^{8}$. There was as expected, a linear increase in water flux with increased pressure difference across the membrane as shown in Figure 3.11 for the AKP-15 supports sintered at 1050,1150 and $1200^{\circ} \mathrm{C}$. This observation is in agreement with data obtained by Steenkamp. ${ }^{10}$ and Kim et al. ${ }^{15}$ The decrease in flux with increasing sintering 
temperature can be attributed to the decrease in porosity and pore diameter with increasing sintering temperature as discussed earlier. The dependence of flux on porosity and pore diameter is illustrated clearly by the Hagen Poiseuille equation. From the gradient of the straight lines of the flux vs. pressure plots (Figure 3.12), the water permeability was calculated and presented as a function of sintering temperature.

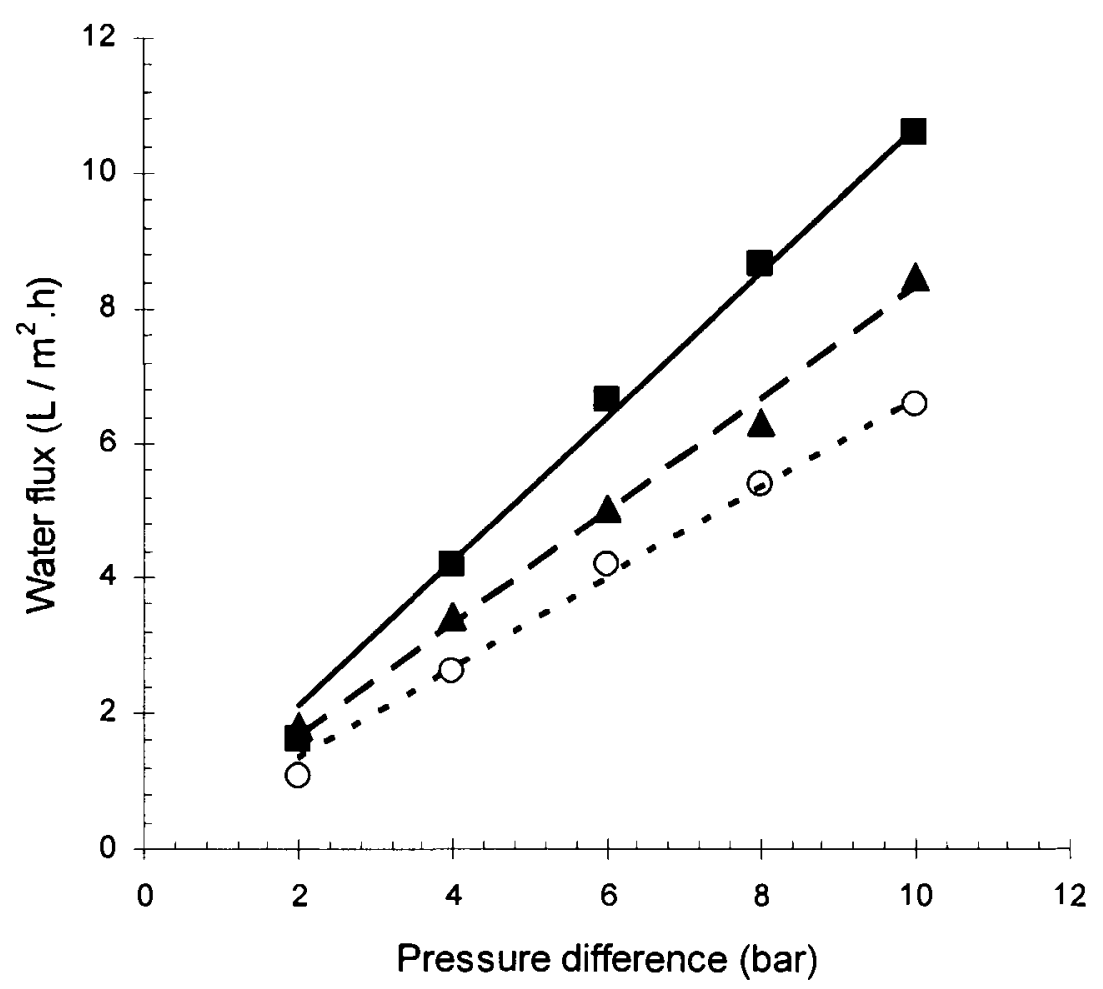

Figure 3.12 Volumetric flow rate of water through AKP-15 supports sintered at different temperatures.

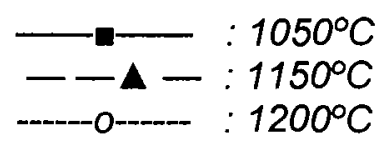

According to Figure 3.13 , there seems to be a linear relationship between particle diameter and water permeability. The permeability decreases when higher sintering temperatures and smaller particles are used. Whilst it seems as though this is a direct linear relationship, too few data points are available to verify such an assumption. Previously it was shown that the particle size had a profound influence on the particle packing and consequently the pore size evolution during sintering. Due to the dependence of permeability on pore radius (Hagen-Poiseuille equation) it is then understandable that the particle size ultimately influences the permeability through the membrane. As illustrated in Figure 3.13, the smaller particles resulted in a decrease in permeability for all the temperatures due to the smaller pore sizes present in these structures. 
The dependence of permeability on sintering temperature is further illustrated in Figure 3.14. According to the Hagen-Poiseuille equation, permeability is directly proportional to porosity. Earlier it was indicated that porosity was largely determined by the temperature at which the support structure was sintered. The higher the sintering temperature, the lower the porosity and thus ultimately the lower the permeability as indicated by the lower permeability of the structures sintered at $1050^{\circ} \mathrm{C}$ vs. the structures sintered at $1200^{\circ} \mathrm{C}$.

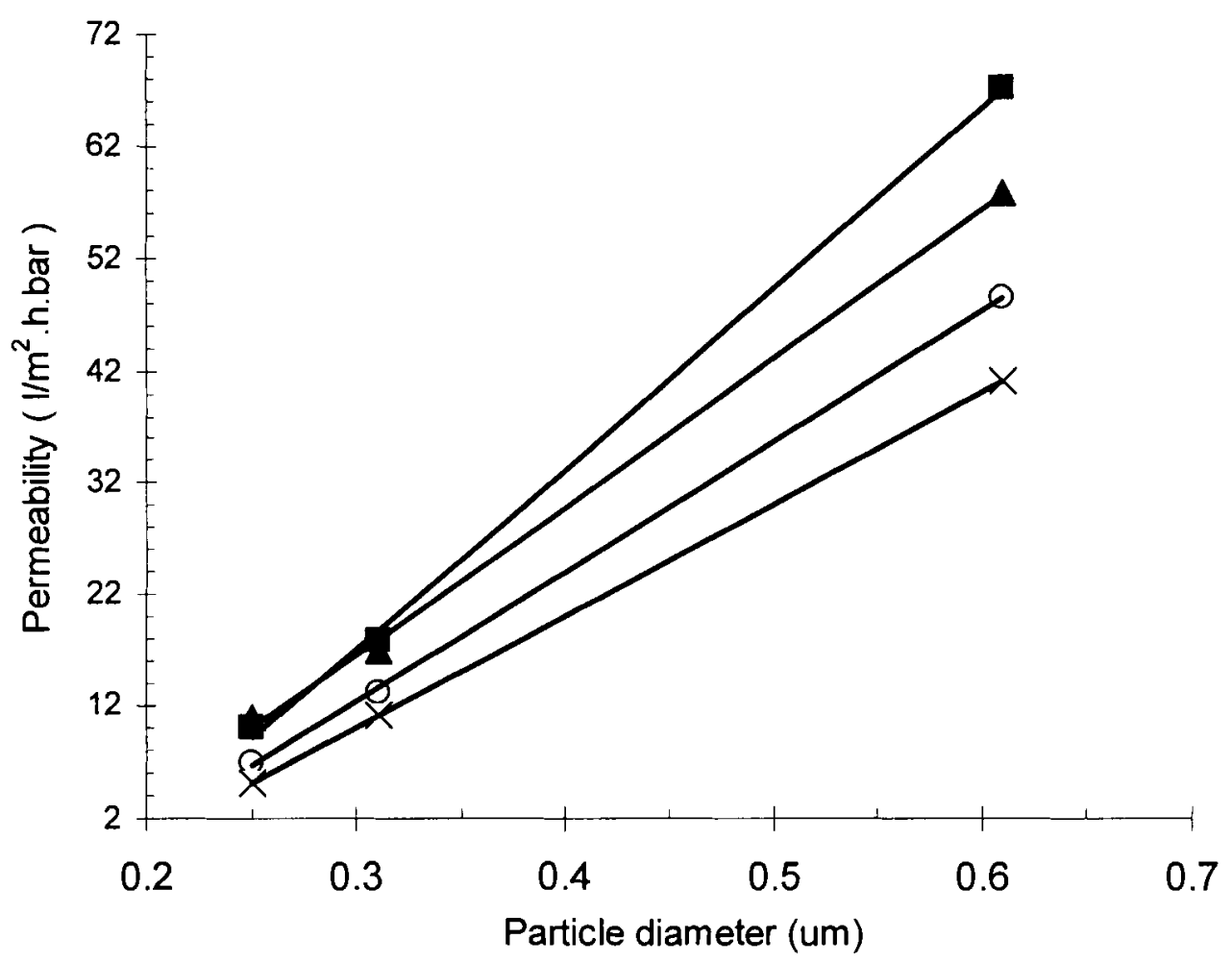

Figure 3.13 Water permeability as a function of particle diameter of starting powder for supports sintered at various temperatures.

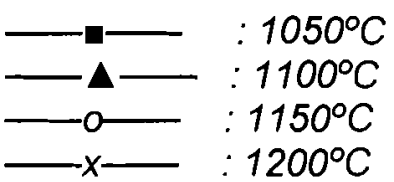

When studying the permeability as a function of the sintering temperature (Figure 3.14), it becomes clear that the larger porosity and pore diameter of the AKP-15 supports resulted in a higher water permeability than the AKP-30 and AKP-50 supports. The increased sintering temperature which produced a microstructure with a lower porosity and smaller pores or a constant pore size (but lower porosity) resulted in a lower water flux as would be expected. This decrease in water permeability with increase in sintering temperature corroborate with results obtained by Kim et al. ${ }^{15}$ 
and Steenkamp et al. ${ }^{16}$ Pure water flux results obtained with the AKP-15 membrane were higher than the pure water flux of tubular membranes presented in literature (see Table3.2). Kim et al. ${ }^{15}$ centrifuged their membranes at 2500 rpm, which possibly resulted in a decreased pore gradient across the membrane wall and thus a decreased flux through the membrane while Steenkamp et al. ${ }^{16}$ used less polymer in their membrane production. A larger amount of polymer was added in this study to create structural defects and thus increase permeability through the membrane. ${ }^{3}$

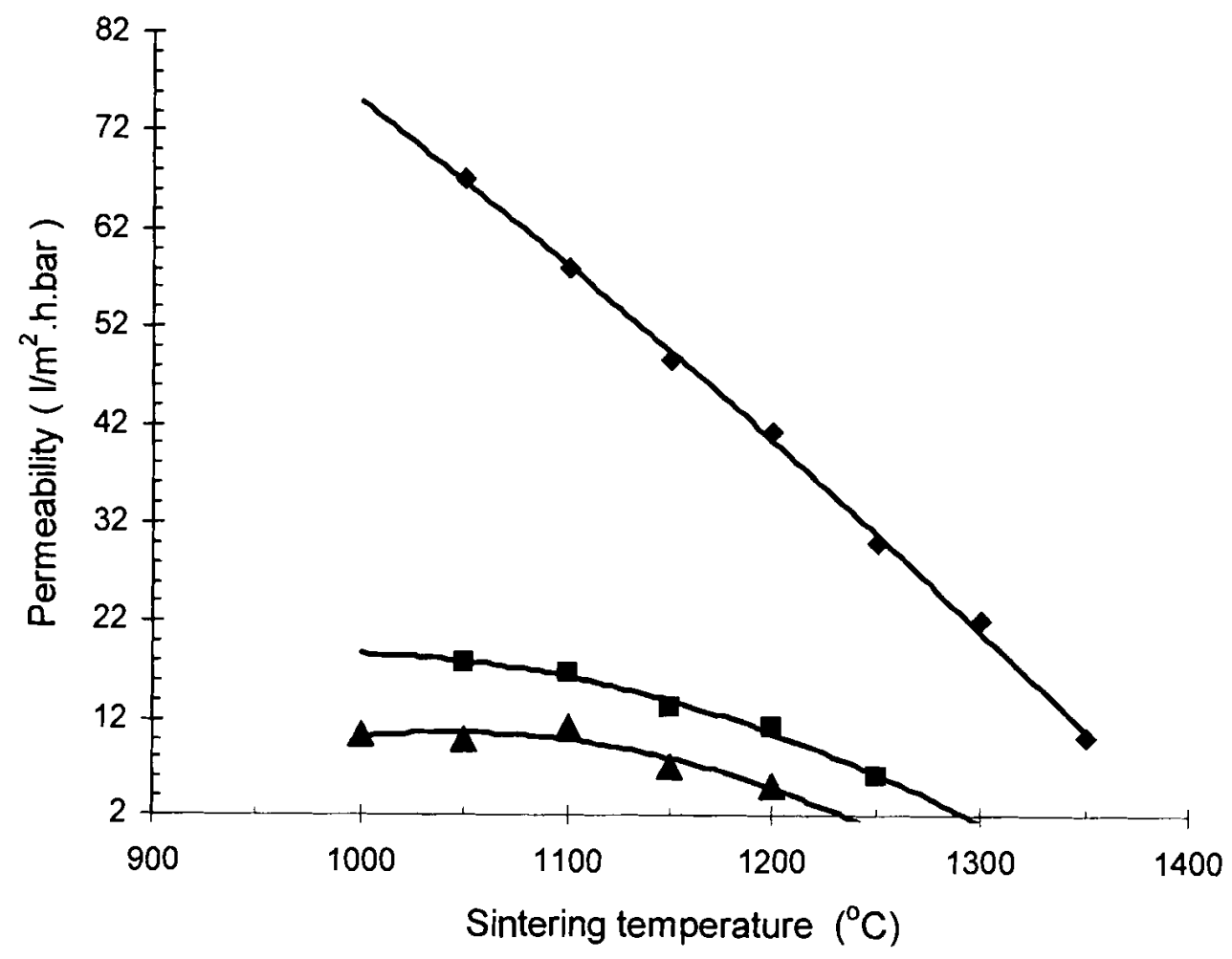

Figure 3.14 Water permeability of three different powders at various temperatures.

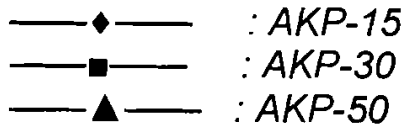


Table 3.2 Comparisons of water fluxes for centrifugally casted ceramic supports

\begin{tabular}{|c|c|c|c|c|}
\hline \multirow{2}{*}{$\begin{array}{l}\text { Particle size } \\
\qquad(\mu \mathrm{m})\end{array}$} & \multicolumn{2}{|c|}{ Flux (L.h. $\left.{ }^{-1} \mathrm{~m}^{-2}\right)$} & \multirow{2}{*}{$\begin{array}{c}\text { Permeability } \\
\text { (L.h. }{ }^{-1} \text { bar. }{ }^{-1} \mathbf{m}^{-2} \text { ) }\end{array}$} & \multirow[t]{2}{*}{ Reference } \\
\hline & 2 bar & 6 bar & & \\
\hline 0.5 & 20 & 73 & - & Kim et al. ${ }^{15}$ \\
\hline 0.8 & 23 & 71 & - & Kim et al. ${ }^{15}$ \\
\hline 0.61 (AKP-15) & - & - & 28 & Steenkamp et al. ${ }^{16}$ \\
\hline $0.61(\mathrm{AKP}-15)$ & 65 & 252 & 41 & This study \\
\hline
\end{tabular}

In Table 3.3 the experimental flux obtained and the theoretical flux (calculated form the HagenPoiseuille equation) are given. The theoretical fluxes were calculated using the porosity and pore diameter values obtained from mercury porosimetry as illustrated in Figure 3.6 and Figure 3.7, respectively. The value for the tortuosity $(\tau)$ was taken as 2.5 and the viscosity of water as 0.001Pa.s for all calculations. In the calculation of the theoretical flux Steenkamp. ${ }^{10}$ made the assumption that $\tau=1 / \varepsilon$. In this study, however, a better correlation was obtained when the tortuosity $(\tau)$ equals 2.5 .

The $\%$ difference between the experimental and the theoretical flux obtained by Steenkamp et al. ${ }^{10}$ was less than the difference calculated here. This could be due to the larger amount of polymer added in this study to increase the porosity, which probably induces "cracks". These "cracks" would be reflected in the increased porosity and mean pore diameter as was seen in Section 3.3.2.2, but the effect on the value of $\tau$ is not indicated. The "cracks" will result in a decrease in the tortuosity $(\tau)$, which would explain why the experimental flux was generally higher than the theoretical flux calculated for $\tau$ equals 2.5. This effect was more profound for supports sintered at lower temperatures as shown in Figure 3.15 where the \% difference between experimental and theoretical fluxes is noticeably higher at lower sintering temperatures for all three particle sizes used. It has previously been shown that the porosity of the support is considerably higher at lower sintering temperatures for all three powder sizes. Profound densification occurred in supports sintered at higher temperatures. The effect of "cracks" would thus be greater; contributing to the higher porosity, at lower temperatures. At higher sintering temperatures the effect of "cracks" is reduced due to the extensive densification. 
Table 3.3 Experimental and theoretical water permeation

\begin{tabular}{|c|c|c|c|}
\hline \multirow[t]{2}{*}{$\begin{array}{l}\text { Powder } \\
\text { Type }\end{array}$} & \multirow[t]{2}{*}{$\begin{array}{c}\text { Temperature } \\
\left({ }^{\circ} \mathrm{C}\right)\end{array}$} & \multicolumn{2}{|c|}{$\begin{array}{c}\text { Permeability } \\
\text { (mol.m. } .^{-2} \text { s. }^{-1} \text { bar. }{ }^{-1} \text { ) }\end{array}$} \\
\hline & & Experimental & Theoretical \\
\hline$K P-15$ & 1050 & 1.035 & 0.708 \\
\hline AKP-15 & 1100 & 0.892 & 0.628 \\
\hline AKP-15 & 1150 & 0.748 & 0.515 \\
\hline AKP-15 & 1200 & 0.634 & 0.483 \\
\hline AKP-15 & 1250 & 0.460 & 0.415 \\
\hline AKP-15 & 1300 & 0.340 & 0.358 \\
\hline AKP-30 & 1050 & 0.292 & 0.206 \\
\hline AKP-30 & 1100 & 0.260 & 0.205 \\
\hline AKP-30 & 1150 & 0.202 & 0.160 \\
\hline AKP-30 & 1200 & 0.170 & 0.149 \\
\hline AKP-30 & 1250 & 0.095 & 0.086 \\
\hline AKP-50 & 1050 & 0.161 & 0.091 \\
\hline AKP-50 & 1100 & 0.170 & 0.099 \\
\hline AKP-50 & 1150 & 0.108 & 0.077 \\
\hline AKP-50 & 1200 & 0.078 & 0.075 \\
\hline
\end{tabular}




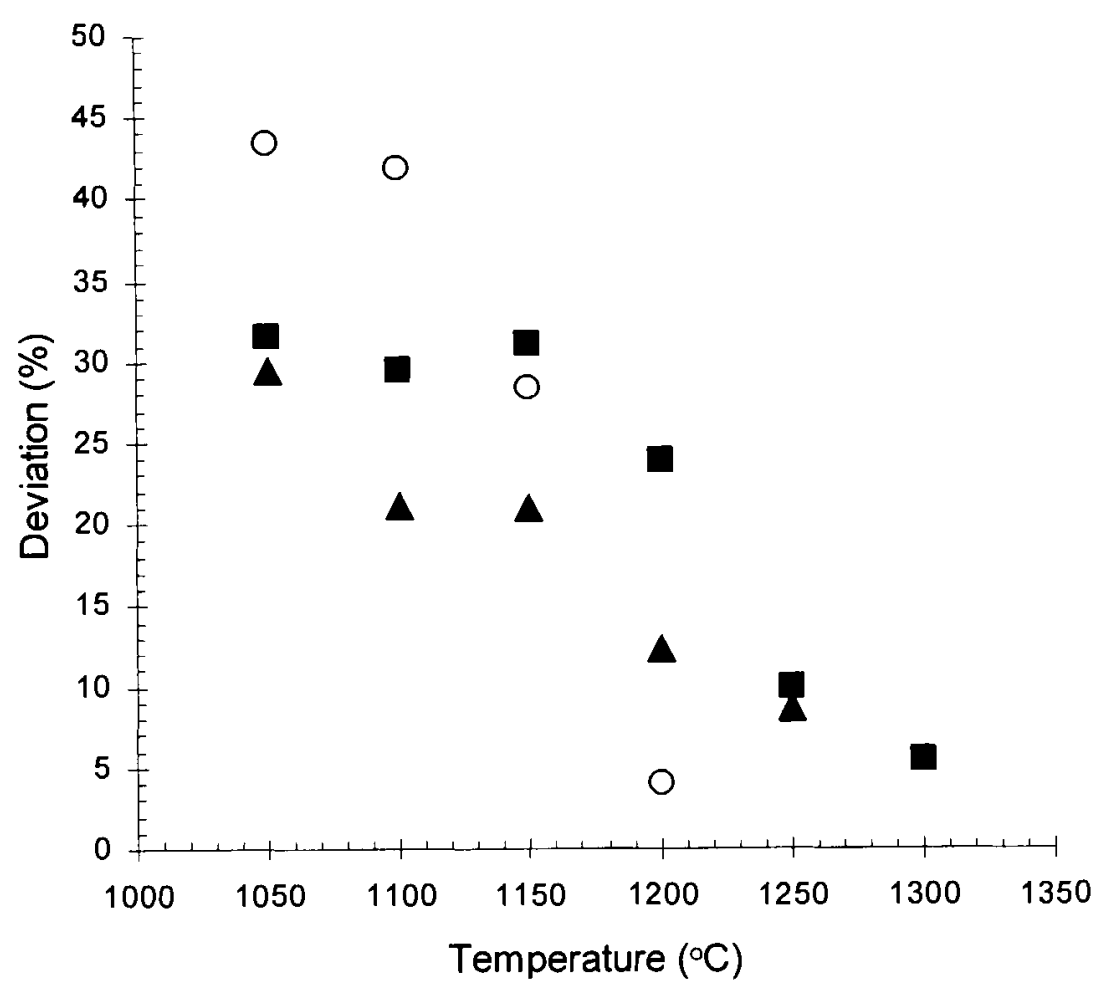

Figure 3.15 Deviation between experimental and theoretical permeation at various temperatures.

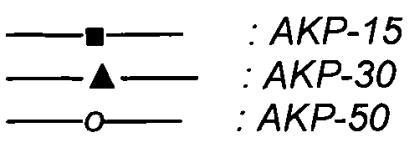

\subsubsection{SEM}

The SEM images of the inner surface of the AKP-50 supports sintered at various temperatures are shown in Figure 3.16. The support surface had a homogeneous particle packing, thus resulting in a very smooth inner surface of the tubular support structure. A slight increase in particle size was observed between $1000^{\circ} \mathrm{C}$ and $1050^{\circ} \mathrm{C}$ at lower temperatures (Figure 3.16a-b), while no particle growth occurred between $1050^{\circ} \mathrm{C}$ and $1100^{\circ} \mathrm{C}$ (Figure $3.16 \mathrm{~b}-\mathrm{C}$ ). However, rapid growth and densification occurred at temperatures above $1100^{\circ} \mathrm{C}$ (Figure $3.16 \mathrm{~d}-\mathrm{e}$ ). The slight increase in grain size between $1000^{\circ} \mathrm{C}$ and $1050^{\circ} \mathrm{C}$ was due to the limited densification and neck growth between individual particles. At these lower temperatures, surface diffusion is dominant, ${ }^{16}$ resulting in a slight decrease in porosity and an increase in linear shrinkage as seen earlier. At higher temperatures, the role of grain boundary diffusion increases, leading to rapid grain growth 
and densification of the compact. This explains the rapid decrease in porosity $(33.83 \%$ to $7.35 \%)$ and increase in linear shrinkage $(5.78 \%$ to $14.24 \%)$ between $1150^{\circ} \mathrm{C}$ and $1300^{\circ} \mathrm{C}$. As can be seen further from the SEM images, the amount of pores decreases as the sintering temperature increases. At $1100^{\circ} \mathrm{C}$ many small pores are visible, but at $1250^{\circ} \mathrm{C}$ only a few remain and at $1300^{\circ} \mathrm{C}$ even fewer can be seen, as would be expected when the visual images are compared to the pore volume as a function of sintering temperature (Figure 3.9). The same trend was observed for the AKP-30 and AKP-15 inner surface SEM images (not presented).

In Figure 3.17, the SEM images of the AKP-50 (a), AKP-30 (b) and AKP-15(c) inner surfaces sintered at $1300^{\circ} \mathrm{C}$ are shown. It is clear that the degree of densification is much higher for the AKP-50 structure than for the AKP-30 at $1300^{\circ} \mathrm{C}$, with the individual particles still visible for the AKP-15 support. This observation confirms the fact that the smaller the initial powder size, the larger the densification and grain growth rates during sintering, confirming the mercury porosimetry results obtained in this study. 


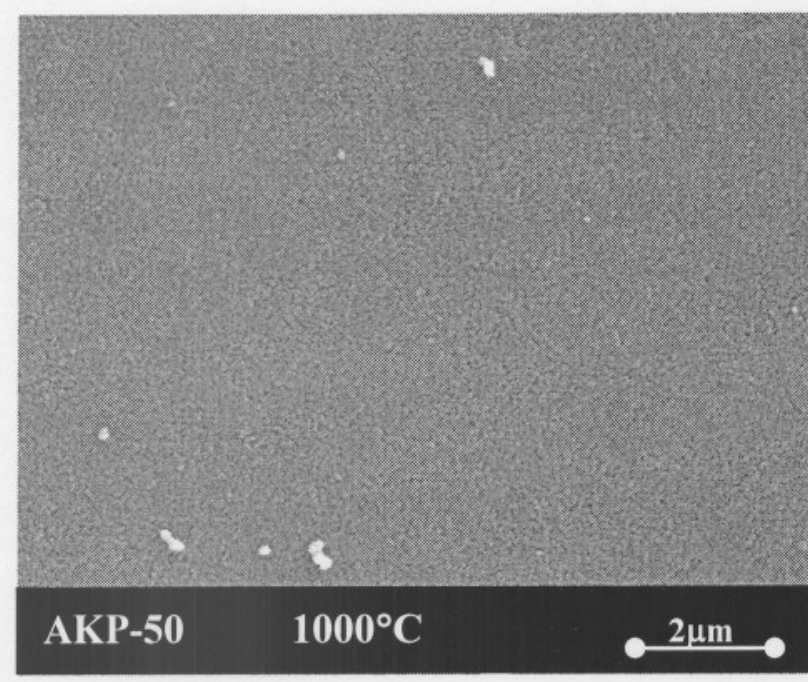

(a)

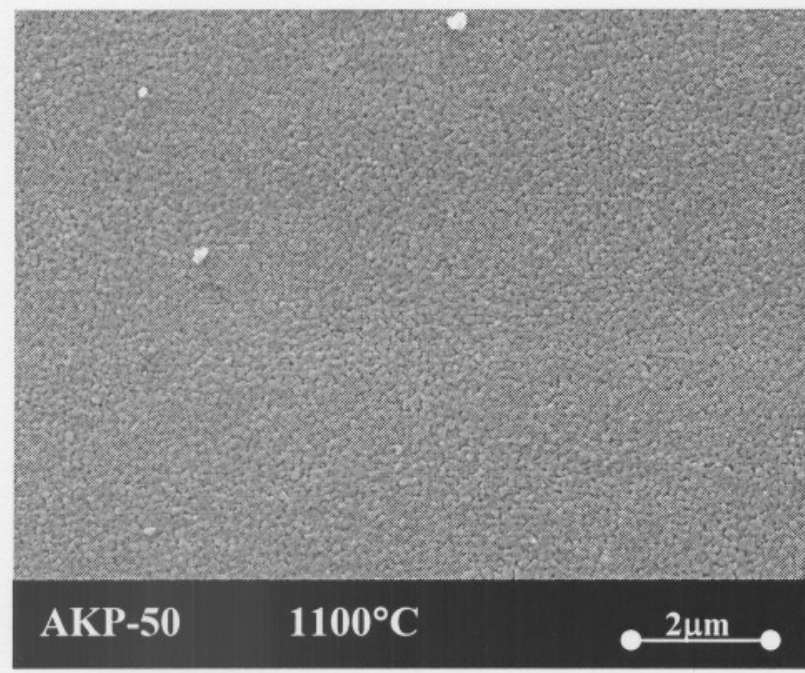

(c)

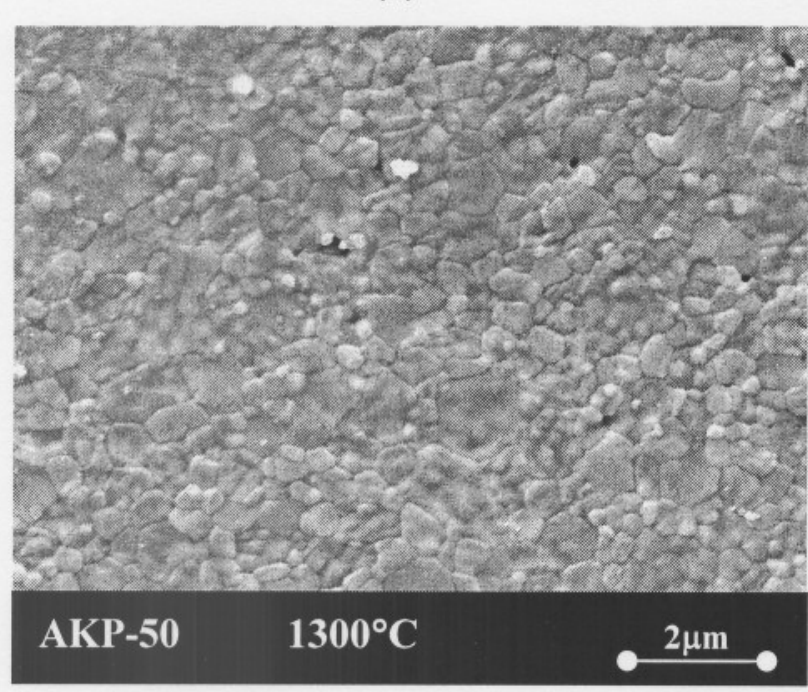

(e)

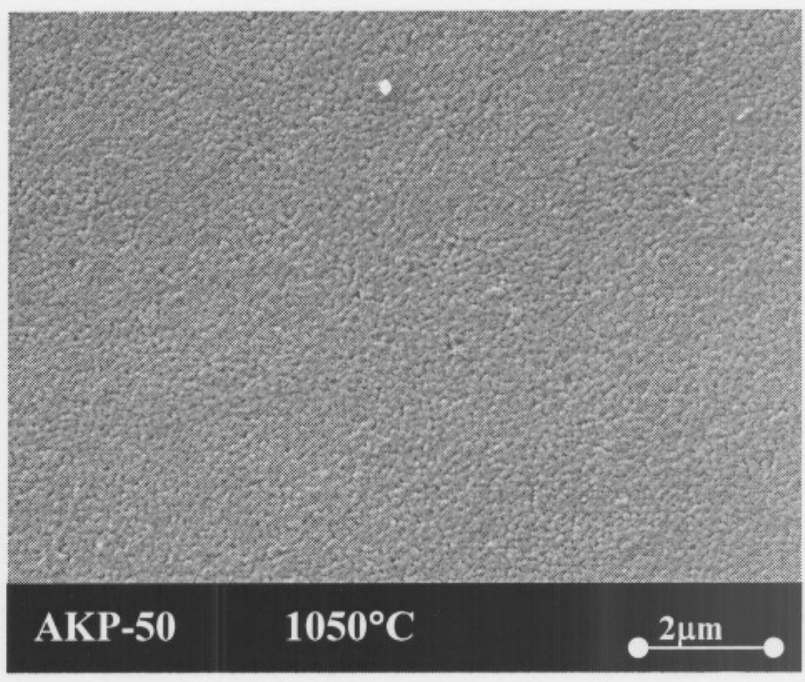

(b)

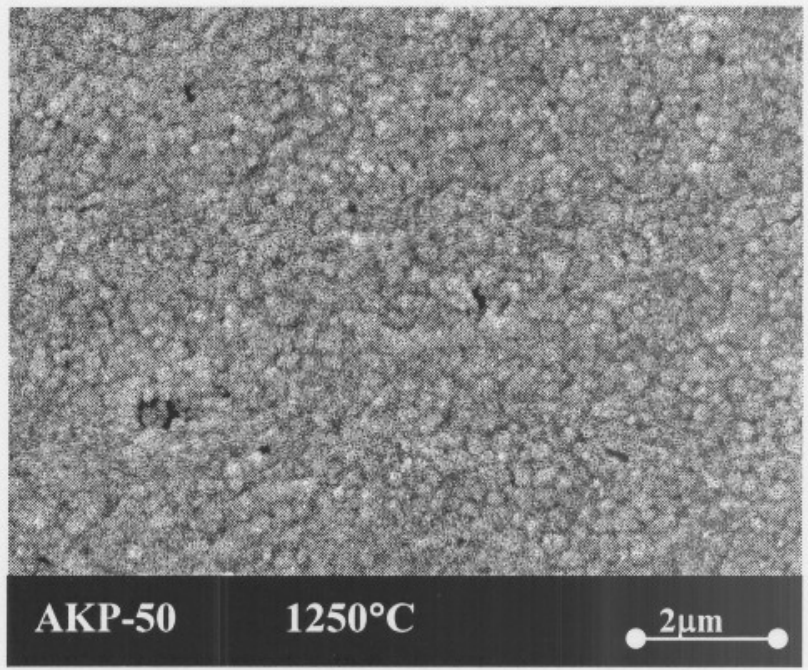

(d)

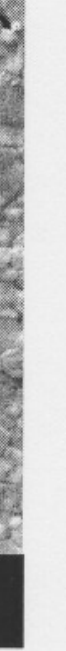




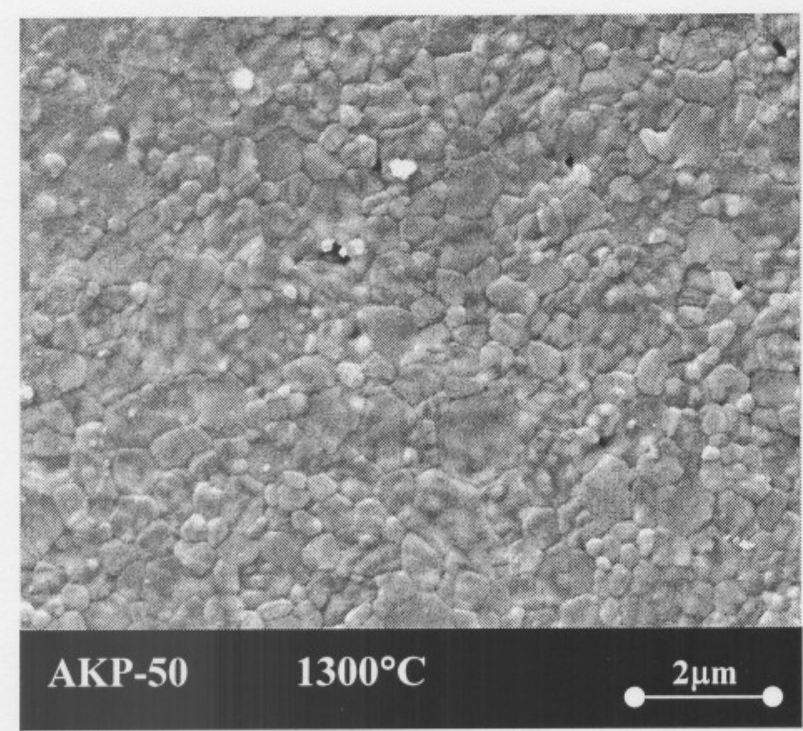

(a)

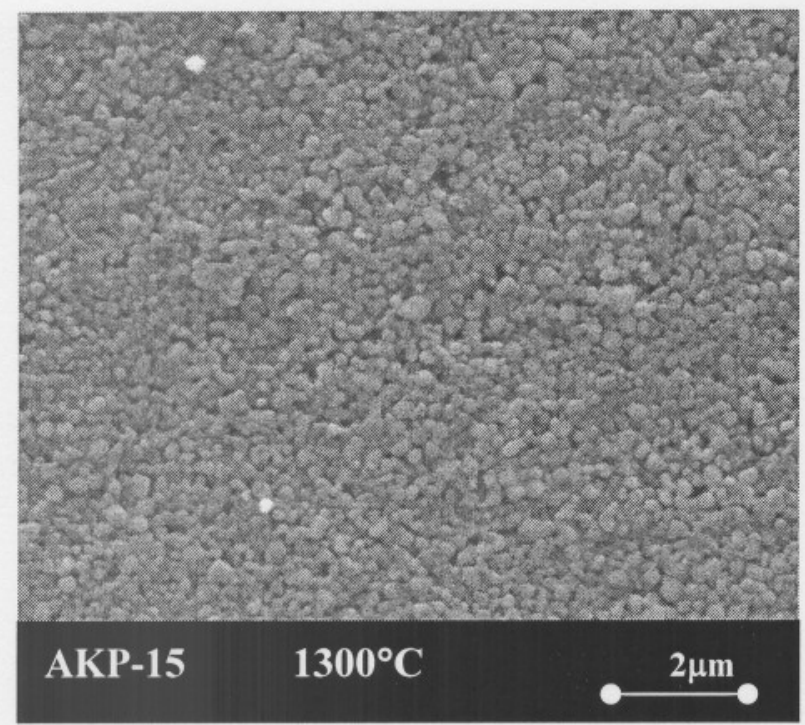

(c)

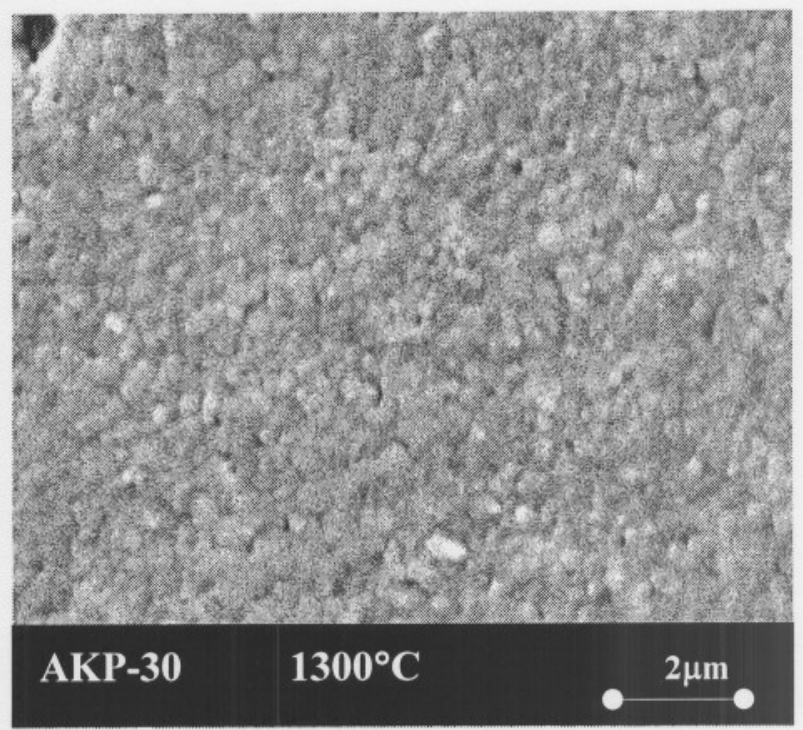

(b)

Figure 3.17 (a) AKP-50, (b) AKP-30 and (c) AKP-15 supports sintered at $1300^{\circ} \mathrm{C}$.

\subsubsection{Mechanical strength}

Most existing methods to determine the strength of a membrane apply pressure on the outside of the tubular structure and only on a very small surface area. The objective of this new method was to evaluate the strength of the structure by applying a force from the inside towards the outside of 
the tubular structure over a larger area, thus observing the fracture toughness of the entire structure. In many studies the liquid or gases to be separated are permeated from the inside through the ceramic support membrane. Evaluating structural strength by exerting pressure from the inside towards the outside would therefore give a better indication of the strength of the support during separation processes.

With the novel mechanical strength test an increase in mechanical strength with increasing sintering temperature and decreasing particle size of the starting powder was observed (Figure 3.18). The higher tensile strength at higher sintering temperatures is due to the increased neck area between particles. The use of the smaller particles (AKP-50) resulted in the highest tensile strength due to the higher number of neighboring particles or necks per unit volume after sintering. The AKP-15 supports thus have a much lower tensile strength due to the fewer neighboring particles or necks per unit volume. ${ }^{10}$ It is known that the tensile strength is also a function of the porosity as the sintering temperature influenced the porosity of the support structure. ${ }^{24} \mathrm{~A}$ decrease in porosity results in an increased tensile strength. Supports with similar porosities had similar tensile strengths as can be seen when the AKP-30 sintered at $1200^{\circ} \mathrm{C}$ is compared to the AKP-50 support sintered at $1150^{\circ} \mathrm{C}$. Both have a strength of $\pm 1800 \mathrm{MPa}$ and a porosity of $\pm 32 \%$. A similar observation was made by Biesheuvel at al. ${ }^{4}$ during their experimental and model evaluation of the tensile strength of ceramic supports done by a dry-pressing method. They made use of the fourpoint-bending-test for the experimental testing. Although their model evaluation indicated an increase in tensile strength with increasing sintering temperature and a decrease in particle size, the experimental data did not confirm these trends. According to the authors this might have been due to the fact that the dry-pressing technique results in flaws in the final product, which resulted in a decrease in the tensile strength of the supports. ${ }^{23}$ Chen et al., ${ }^{24}$ who used the diametral compressing testing, also found increased fracture strength with decreasing porosity. Flinn et al. ${ }^{25}$ made use of the Archimedes method to obtain failure strengths for compacts with various densities between 60 and $100 \%$. The authors also observed that there was an increase in failure strength with increased density (decrease in porosity) of the porous alumina structures. According to their results, however, the relation was not linear. 


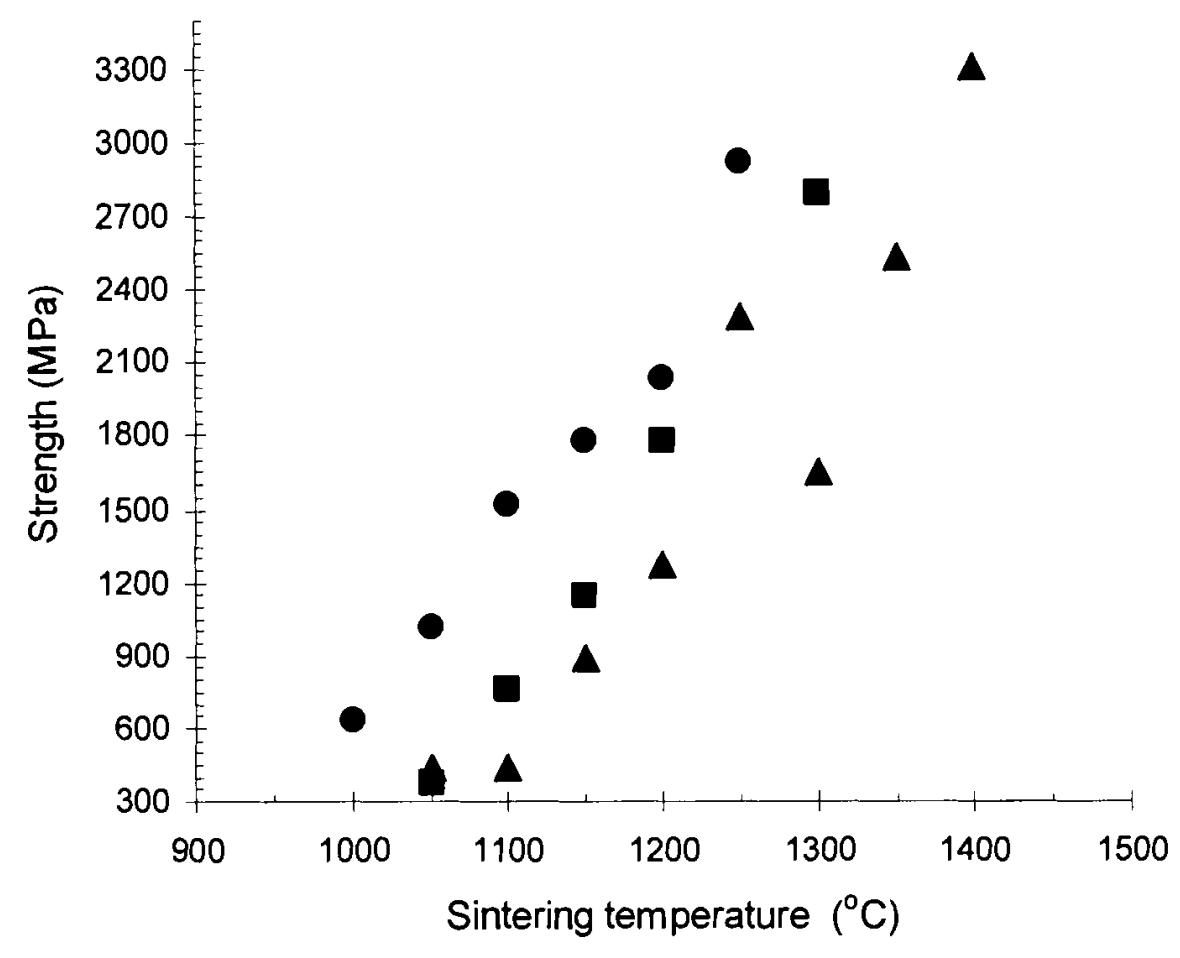

Figure 3.18 Mechanical strength of various membranes sintered at different temperatures.
$\Delta: A K P-15$
- :AKP-30
- $: A K P-50$

The inversely proportional relation between strength and porosity is confirmed when strength is plotted against porosity for all three powder sizes investigated in this study (as illustrated in Figure 3.19). According to the best fit, it seems that the relation between strength and porosity is linear for the AKP-50 and polynomial for AKP-15 and AKP-30. However, more data points would be required to confirm the tendencies. 


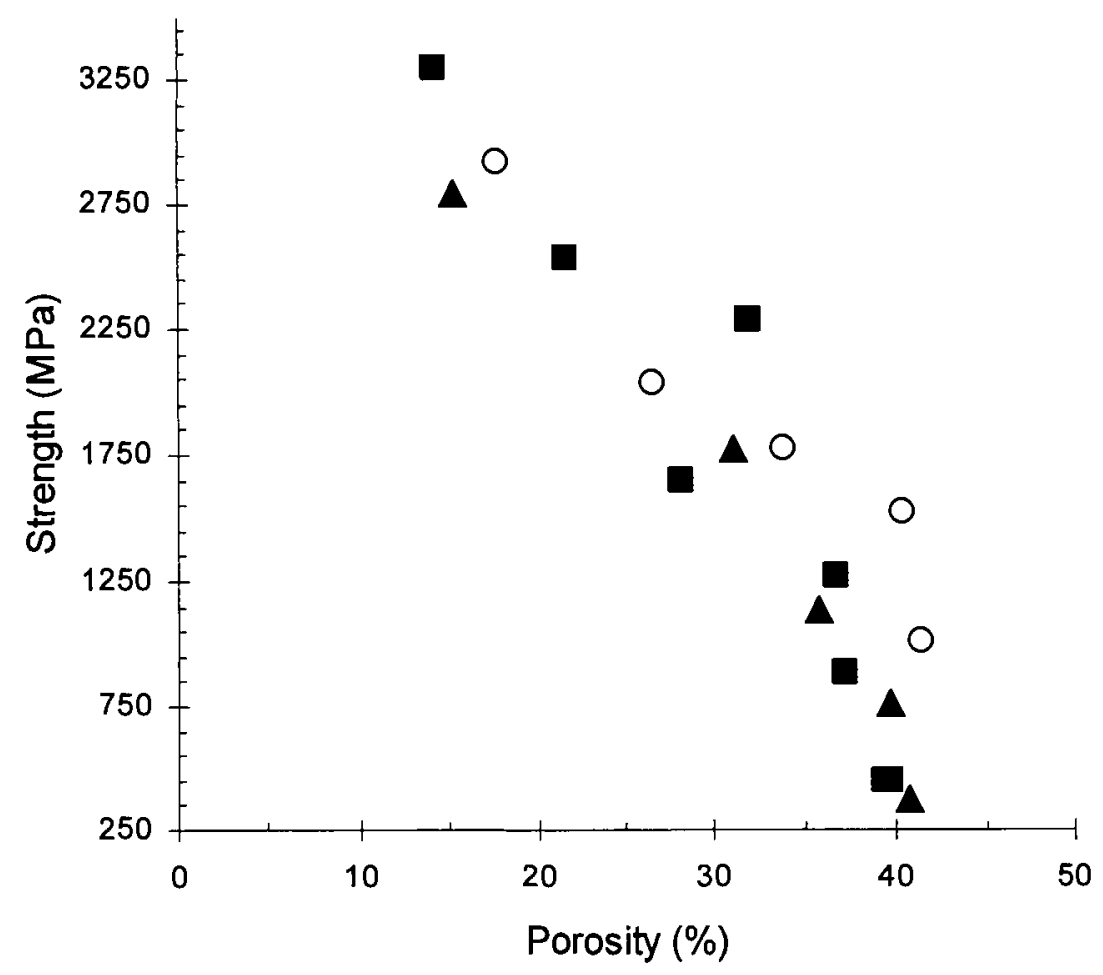

Figure 3.19 Mechanical strength of the various membrane porosities.
$\Delta: A K P-15$
- $: A K P-30$
$0: A K P-50$

\subsubsection{Influence of dispersant concentration and heating rate}

\subsubsection{Support dimensions and linear shrinkage}

The amount of polymer added to the starting suspension of the AKP-30 powder sintered at $1100^{\circ} \mathrm{C}$ as well as the heating rate from room temperature to $400^{\circ} \mathrm{C}$ had no noticeable effect on the dimensions or the linear shrinkage of the support structures as can be seen from Table 3.4. 
Table 3.4 Influence of the amount of polymer and the heating rate on the dimensions of the AKP-30 support sintered at $1100^{\circ} \mathrm{C}$

\begin{tabular}{ccccc}
\hline $\begin{array}{c}\text { Heating rate } \\
\text { to } \mathbf{4 0 0} 0^{\circ} \mathrm{C} \\
\left({ }^{\circ} \mathrm{C} / \mathbf{m i n}\right)\end{array}$ & $\begin{array}{c}\text { Amount of } \\
\text { polymer } \\
(\mathbf{w t} \%)\end{array}$ & $\begin{array}{c}\text { Outer diameter } \\
(\mathbf{m m})\end{array}$ & $\begin{array}{c}\text { Inner diameter } \\
(\mathbf{m m})\end{array}$ & $\begin{array}{c}\text { Linear shrinkage } \\
(\%)\end{array}$ \\
\hline 0.4 & 5.48 & 20.75 & 17.80 & 1.51 \\
0.4 & 10.48 & 20.80 & 17.75 & 1.71 \\
1.0 & 5.48 & 20.75 & 17.90 & 1.65 \\
1.0 & 10.48 & 20.70 & 17.80 & 1.61 \\
\hline
\end{tabular}

\subsubsection{Mercury porosimetry}

While the amount of polymer had little effect on the shrinkage, it did alter the properties of the support structures as can be seen from Table 3.5. The higher the amount of polymer added, the higher the porosity and median pore diameter for the AKP-30 supports sintered at $1100^{\circ} \mathrm{C}$. Benito et $a l^{3}$ made use of this method to increase the porosity of their membranes by adding a higher amount of PVA in their starting suspension. According to Benito et al., ${ }^{3}$ the higher the amount of polymer, the more agglomerates are formed in the suspension, which in turn creates defects during drying, resulting in an increase in porosity after sintering. The higher heating rate had no noticeable effect on the porosity or median pore size of the supports in this study. Zhou et al. ${ }^{26}$ made use of pulse electric current sintering to obtain very high sintering rates. They stated that, under certain conditions, rapid heating could enhance densification. In their study they observed that fast heating reduced grain growth, especially for the smaller powder sizes.

In Figure 3.20, the pore volume as a function of the pore size is shown for the membranes manufactured with varied amounts of polymer and at different heating rates. 
Table 3.5 Influence of the amount of polymer and the heating rate on the properties of the AKP-30 supports sintered at $1100^{\circ} \mathrm{C}$

\begin{tabular}{ccccc}
\hline $\begin{array}{c}\text { Heating rate } \\
\text { to } \mathbf{4 0 0} 0^{\circ} \mathrm{C} \\
\left({ }^{\circ} \mathrm{C} / \mathbf{m i n}\right)\end{array}$ & $\begin{array}{c}\text { Amount of } \\
\text { polymer } \\
(\mathbf{w t} \%)\end{array}$ & $\begin{array}{c}\text { Porosity } \\
(\%)\end{array}$ & $\begin{array}{c}\text { Pore diameter } \\
(\mathbf{n m})\end{array}$ & $\begin{array}{c}\text { Water } \\
\text { permeability } \\
\left(\mathbf{L} . \mathbf{h}^{-1} \cdot \mathbf{b a r}^{-1} \cdot \mathbf{m}^{-2}\right)\end{array}$ \\
\hline 0.4 & 5.48 & 37.77 & 88.25 & 10.67 \\
0.4 & 10.48 & 39.25 & 92.70 & 12.05 \\
1.0 & 5.48 & 37.74 & 88.95 & 10.37 \\
1.0 & 10.48 & 39.46 & 92.60 & 12.94 \\
\hline
\end{tabular}

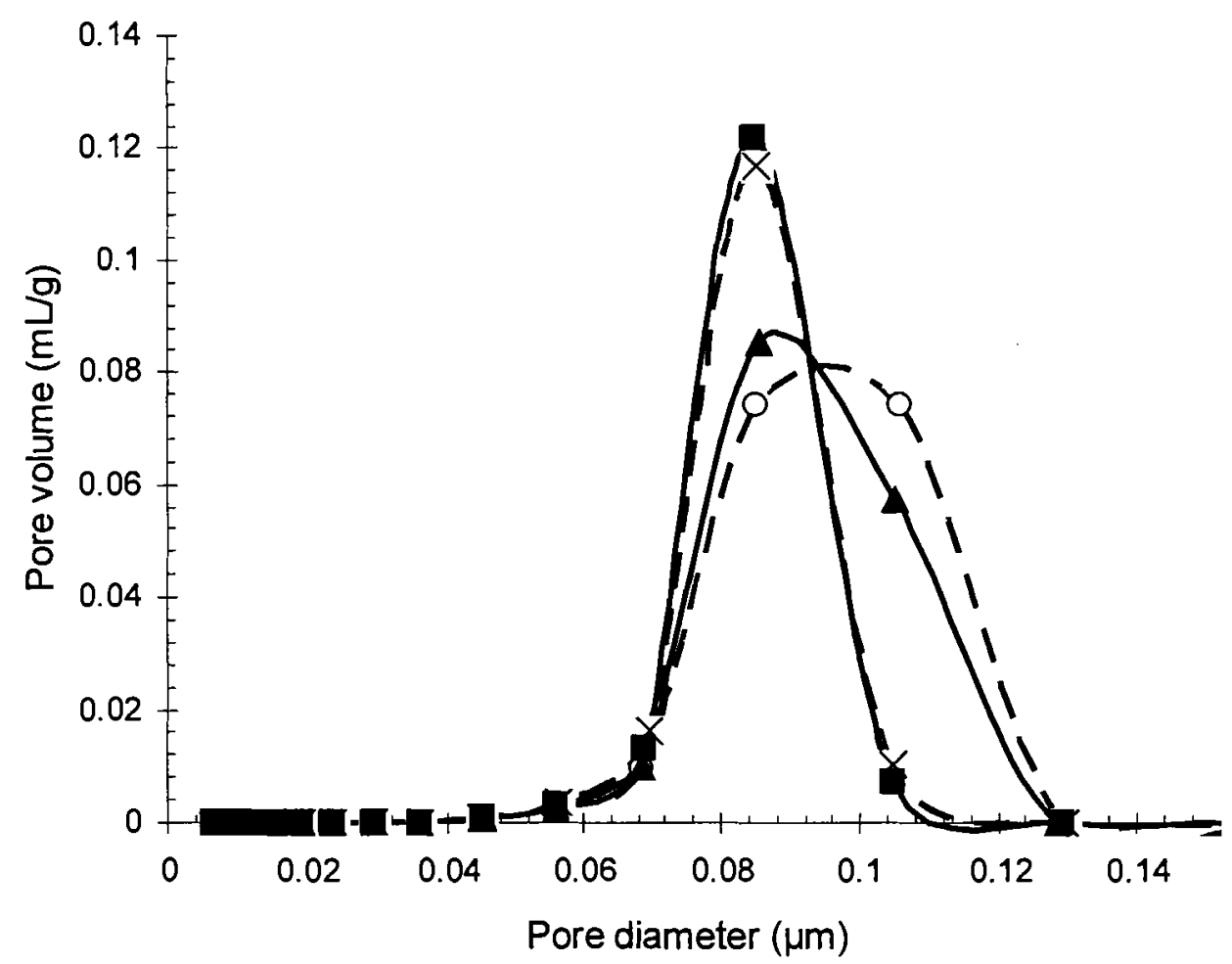

Figure 3.20 Pore volume as a function of pore diameter for the AKP-30 supports sintered at $1100^{\circ} \mathrm{C}$ with varied amounts of polymer and heating rate

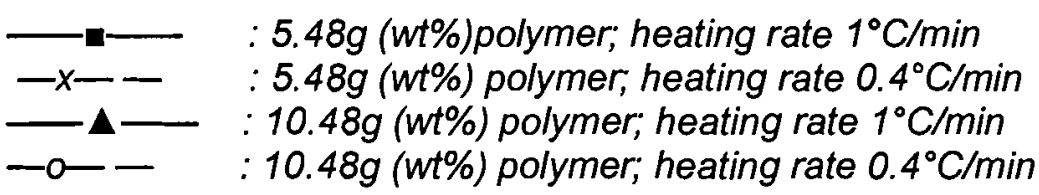


The supports with less polymer (APMA) had a smaller median pore diameter as was seen in Table 3.5. The median pore diameter increased when the amount of polymer was increased, which also led to a broadening of the pore size distribution towards the larger pore sizes. Although this shift in pore size distribution was combined with a decrease in the pore volume of the median pore size it contributed to an increased porosity as shown in Table 3.5. The increased porosity was therefore due to the larger pores being produced during the sintering of the powder compact. The larger "pores" might be cracks or defects being created as Benito et al. ${ }^{3}$ suggested. Both the polymer concentrations used in this study were substantially higher than the amount necessary for a suspension with an optimal dispersion. According to Nijmeijer et al., ${ }^{27}$ the optimum results for a defect free support were $167 \mathrm{~kg} / \mathrm{m}^{3}$ and $83 \mathrm{~kg} / \mathrm{m}^{3}$ of Darvan $\mathrm{C}$ dispersant for the AKP-30 and AKP15 supports respectively. Any amount less or more would generate cracking and/or warping depending on the quantity of polymer added to the starting suspension. In this study we used 219 $\mathrm{kg} / \mathrm{m}^{3}(10.48 \mathrm{wt} . \%)$ and $109 \mathrm{~kg} / \mathrm{m}^{3}(5.48 \mathrm{wt} . \%)$ of Darvan $\mathrm{C}$ to produce the supports. This increase in porosity due to "defects" would also help explain the above average water permeabilities obtained in this study.

\subsubsection{Water permeability}

The increased amount of polymer affected both the porosity and pore diameter as was shown in Section 3.3.3.2. As the water permeability is a function of porosity and pore diameter, ${ }^{8}$ the increased amount of polymer resulted in an increased water permeability as is clear from Table 3.5. The increased amount of polymer from 5.48 to $10.48 \mathrm{wt} \%$ resulted in a $\approx 16 \%$ increase in the water permeability for both the $0.4^{\circ} \mathrm{C} / \mathrm{min}$ and $1.0^{\circ} \mathrm{C} / \mathrm{min}$ heating rates. The heating rate had no noticeable effect on the permeability of the support structure in this study.

\subsubsection{SEM}

As there was only a slight change in porosity and pore diameter, no noticeable difference could be observed in the SEM images between membranes manufactured with different amounts of polymer or sintered at various rates. 


\subsubsection{Mechanical strength}

As the amount of APMA (polymer) was increased, the porosity increased and consequently the tensile strength of the support structure decreased independently of the heating rates (Table 3.6). Increasing the heating rate resulted in a slight decrease in tensile strength for the supports with more APMA, but remained constant for the supports with less APMA. It is interesting that the porosity of the AKP-30 supports (with less polymer) was very similar to the porosity of the AKP-15 supports sintered at $1150^{\circ} \mathrm{C}$ (with more polymer) according to Figure 3.19. Again it is found that, when comparing these two membranes, the tensile strength is more or less equivalent.

This confirms that the tensile strength depends largely on the porosity of the support. As mentioned earlier, the porosity of the tubular membrane supports produced by Nijmeijer et al. ${ }^{27}$ was influenced by the amount of polymer added to the starting suspension. When more polymer than necessary was added, cracks and warping of the tube structure occurred with a simultaneous increase in porosity. Defects such as cracking and the increased porosity would ultimately result in lower tensile strength as seen in Table 3.6.

Table 3.6 Influence of the amount of polymer and the heating rate on the tensile strength of the AKP-30 supports sintered at $1100^{\circ} \mathrm{C}$

\begin{tabular}{ccc}
\hline $\begin{array}{c}\text { Heating rate to } 400^{\circ} \mathrm{C} \\
\left({ }^{\circ} \mathrm{C} / \mathrm{min}\right)\end{array}$ & $\begin{array}{c}\text { Amount of polymer } \\
(\mathbf{w t} \%)\end{array}$ & $\begin{array}{c}\text { Tensile strength } \\
(\mathbf{M P a})\end{array}$ \\
\hline 0.4 & 5.48 & 1013.28 \\
0.4 & 10.48 & 759.96 \\
1.0 & 5.48 & 1013.28 \\
1.0 & 10.48 & 633.30 \\
\hline
\end{tabular}




\subsection{Conclusions}

The centrifugal casting technique was used to produce membrane supports of repeatable and high quality. Round asymmetrical supports with a smooth inner surface were manufactured using different powder particle sizes which were sintered at various temperatures. The following conclusion can be made from this study:

- Supports sintered at the lowest sintering temperatures had the highest porosity, water permeability and largest pore sizes while the tensile strength was the lowest for all particle sizes used.

- The use of the large AKP-15 $(0.61 \mu \mathrm{m})$ powder resulted in supports with the highest porosity and water permeability at equivalent sintering temperatures compared to the AKP-30 $(0.31) \mu \mathrm{m}$ and AKP-50 $(0.25 \mu \mathrm{m})$ powders. The tensile strength of the AKP-15 support structures was lower and the mean pore size was larger. The AKP-50 supports had the lowest porosity but highest tensile strength.

- The pore size and pore size distribution was influenced by the starting powder particle size and thus the particle packing during centrifugation as could be seen from the fact that the pore size of the AKP-50 supports remained fairly constant with increasing sintering temperature. This was not the case for the larger particle sizes. The sintering temperature largely determined the porosity of the supports.

- The tensile strength of the support structures was determined by the porosity of the supports. The higher the porosity, the lower the tensile strength.

- The amount of polymer added to the starting suspension influenced the properties of the support structure. Using more polymer resulted in higher water permeability, porosity and pore sizes. The increased amount of polymer resulted in an increase in pore size distribution and lowering of tensile strength. The larger amount of polymer seem to result in the formation of "cracks" and "defects" during sintering.

- The heating rate had no noticeable effect on the membrane support structure. 


\subsection{References}

1 J.Hofman-Züter, Chemical and thermal stability of (modified) mesoporous ceramic membranes, Ph.d. Thesis, University of Twente, Nederlands, 1995, Chapter 2, Theoretical background, pp. 9

${ }^{2}$ W.Zeng, L.Goa, L.Gui, J.Guo, Sintering kinetics of $\alpha-\mathrm{Al}_{2} \mathrm{O}_{3}$, Ceramics International, 25, 1999, 723

3 J.M.Benito, A.Conesa, F.Rubio, M.A.Rodriguez, Preparation and characterazation of tubular ceramic membranes for treatment of oil emulsions, Journal of the European Ceramic Society, 25, 2004, 1895

${ }^{4}$ P.M.Biesheuvel, V.Breedveld, A.P.Higler, H.Verweij, Graded membrane supports produced by centrifugal casting of a slightly polydisperse suspension, Chemical Engineering Science, 56, 2001, 3517

5 G.T.P.Mabande, G.Pradhan, C.Schwieger, M.Hanebuth, R.Dittmeyer, T.Selvam, A.Zampieri, H.Baser, R.Herrmann, A Study of silicalite-1 and Al-ZSM-5 membrane synthesis on stainless steel supports, Microporous and Mesoporous Materials, 75, 2004, 209

6 J.Caro, M.Noack, P.Kölsch, R.Schäfer, Zeolite membranes - state of their development and perspective, Microporous and Mesoporous Materials, 38, 1999, 3

${ }^{7}$ G.A.Gogotsi, Fracture toughness of ceramic and ceramic composites, Ceramics International, 29, 2003,777

${ }^{8}$ P.M.Biesheuvel, Porous ceramic membranes suspension processing, mechanical and transport properties, and application in the osmotic tensiometer, Ph.d. Thesis, Universiteit Twente, The Nederlands, 1999, Summary 
9 P.M.Biesheuvel, H.Verweij, Microdivers to study sedimentation in polydisperse concentrated colloidal suspensions, A/ChE Journal, 47(9), 2001,1969

${ }^{10}$ G.C.Steenkamp, Centrifugal casting and coating of tubular ceramic membrane supports, M.Sc. Thesis, Potchefstroom University for Christian Higher Education, South Africa, 2000, Chapter 2: Centrifugal casting, pp. 25

${ }^{11}$ A.R. Boccaccini, P.A. Trusty, In Situ Characterization of the Shrinkage Behavior of Ceramic Powder Compacts during Sintering by Using Heating Microscopy, Material Characterization, 41, 1998,109

${ }^{12}$ A. Shui, N. Uchida, K. Uematsu, Origin of shrinkage anisotropy during sintering for uniaxially pressed alumina compacts, Powder Technology, 127, 2002, 9

${ }^{13}$ W.D. Kingery, H.K. Bowen, D.R. UhImann, Introduction to Ceramics, Second Edition, Chapter 10, Grain growth, sintering and verification, Wiley Interscience, 1976, pp. 469

${ }^{14}$ Y. Chiang, D. Birnie, W. Kingery, Physical ceramics: Principles for ceramic Science and engineering, Wiley, 1997, Chapter 5, Single-Phase sintering, 403

${ }^{15}$ K. Kim, S. Cho, K. Yoon, J. Kim, J. Ha, D. Chun, Centrifugal casting of alumina tube for membrane application, Journal of Membrane Science, 199, 2002, 69

${ }^{16}$ G.C. Steenkamp, A. Nijmeijer, H.M. Krieg, K. Keizer, Centrifugal casting of ceramic membrane tubes and coating with chitosan, Separation and Purification Technology, 25(1), 2001, 407

17 S.L. Kang, Y. Jung, Sintering kinetics at final stage sintering: model calculation and map construction, Acta Materialia, 52, 2004, 4573 
${ }^{18}$ K. Darcovich, D. Roussel, F.N. Toll, Sintering effects related to filtration properties of porous continously gradient ceramic structures, Journal of Membrane Science, 183, 2001, 293

19 Y. Chiang, D. Birnie, W. Kingery, Physical ceramics: Principles for ceramic Science and engineering, Wiley, 1997, Chapter 5, Single-Phase sintering, 409

${ }^{20}$ P. Wang, P. Huang, N. Xu, J. Shi, Y.S. Lin, Effects of sintering temperature on properties of alumina microfiltration membranes, Journal of Membrane Science, 155, 1999, 309

${ }^{21}$ P.M. Biesheuvel, Henk Verweij, Microdivers to study sedimentation in polydisperse concentrated colloidal suspensions, A/ChE Journal, 47(9), 2001, 1969

${ }^{22}$ N.Das, H.S.Maiti, Effect of size distribution of the starting powder on the pore size and its distribution of tape cast alumina microporous membranes, Journal of the European Ceramic Society, 19, 1999, 341

${ }^{23}$ P.M. Biesheuvel, Henk Verweij, Design of ceramic membrane supports: permeability, tensile strength and stress, Joumal of Membrane Science, 156, 1999, 141

${ }^{24}$ C.H. Chen, K. Takita, S.Honda, H.Awaji, Fracture behaviour of cylindrical alumina with pore gradient, Journal of the European Ceramic Society, 25, 2005, 385

${ }^{25}$ B.D.Flinn, R.K.Bordia, A.Zimmermann, J.Rodel, Evolution of defect size and strength of porous alumina during sintering, Journal of the European Ceramic Society, 20, 2000, 2561

${ }^{26}$ Y.Zhou, K.Hirao, Y.Yamauchi, S.Kanzaki, Effects of heating rate and particle size on pulse electric current sintering of alumina, Scripta Materialia, 48, 2003, 1631

${ }^{27}$ A. Nijmeijer, C. Huiskes, N.G.M. Sibelt, H. Kruidhof and H. Verweij, Centrifugal casting of tubular membrane supports, American Ceramic Society Bulletin, 77, 1998, 95 


\section{Chapter 4}

\section{Manufacture of \\ ceramic supports \\ using Alcoa \\ powder}

\subsection{Introduction}

An important property of ceramics is that they are brittle and fracture with little deformation. This behavior is in contrast to metals which yield and deform without fracturing. As a result, shaping methods for ceramic products can be difficult and costly, which is one of the main reasons why inorganic membrane production is so expensive. ${ }^{1}$ When producing ceramic membranes using a colloidal suspension, the particle size distribution (PSD) has a profound influence on the properties of the colloidal suspension. The PSD influences the viscosity and ultimately the density and particle packing of the green cast being formed. ${ }^{2}$ it has also been shown that a compact made 
from a broader PSD gives a higher green density and improved densification during initial stage sintering. ${ }^{3}$ The pore size distribution is also influenced by the PSD. A narrow PSD results in a narrow pore size distribution, while a wide PSD would result in a wider pore size distribution. ${ }^{4}$ The PSD further more has an effect on the sintering rate of a compact. As illustrated in Figure 4.1 the relative sintering rate is influenced by the size of the particles as well as the pair arrangement. ${ }^{5}$

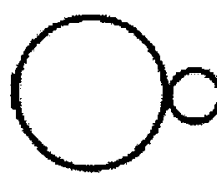

Fastest

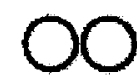

Intermediate

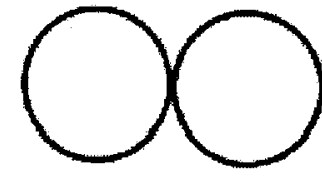

Slowest

Figure 4.1 Influence of pair arrangements on sintering rate.

During the manufacture of graded tubular ceramic supports, for example by centrifugal casting, the PSD and the chemical impurities in the starting powder are of great importance. If agglomerates are present in the colloidal suspension, it would result in flaws in the support structure during sintering. A large PSD can result in "cracks" or warping of the support structure as the large difference in the particle sizes results in varying sintering rates in the various regions of the asymmetric membranes. With centrifugal casting the larger particles are deposited on the outside and the smaller particles on the inside wall of the green cast. During sintering the difference in sintering rates between the various particle sizes could result in the smaller particles on the inside tearing away from the larger particles on the outside if the shrinkage rate difference is too large. In general, a broader PSD is expected to yield a denser green cast and may cause increased structural inhomogeneity as a result of size segregation. ${ }^{3}$

Most commercial $\alpha$-alumina powders available contain impurities. The impurities mostly consist of $\mathrm{Na}_{2} \mathrm{O}, \mathrm{SiO}_{2}, \mathrm{Fe}, \mathrm{CaO}, \mathrm{MgO}, \mathrm{Cl}, \mathrm{K}_{2} \mathrm{O}$, and $\mathrm{Ti}$. Since different powders are manufactured in different manners, the amount and type of impurities can differ. The impurities have an effect on the surface chemistry of the powder particles and thus the adsorption of the dispersion agent (for example APMA) and the zeta-potential of a colloidal suspension. Due to the extensive buffering tendency of impurities, powders containing more impurities are more difficult to stabilize electrostatically. The unstable $\mathrm{pH}$-region is also broadened due to the presence of these impurities. ${ }^{6}$

The use of a chemically pure $\alpha$-alumina powder with a narrow PSD would avoid many of the above mentioned problems concerning the manufacturing of a ceramic support. However, the price of 
such powders is very high. It would therefore be interesting to investigate the possibility of reducing the cost by using cheaper starting powders.

It was the aim of this study to investigate the possibility of replacing the expensive AKP powder range (Sumitomo Chemical Company, Ltd., Japan) with the less expensive Alcoa CT 3000 SG $\alpha$ alumina powder (Alcoa World Chemicals, USA).

\subsection{Experimental}

In this study Alcoa CT3000 SG (Alcoa World Chemicals, USA) was used as an alternative to the more expensive AKP powder range (Sumitomo Chemical Company, Ltd., Japan). According to the manufacturer, the Alcoa $\alpha$-alumina powder has a specific surface area of $7.5 \mathrm{~m}^{2} / \mathrm{g}$ and a chemical purity of $99.8 \%$. According to the manufacturer the PSD is: D50 $-0.8 \mu \mathrm{m}$ and D90 $-2.5 \mu \mathrm{m}$. In literature, variations on these values are found, for example Studart et al. ${ }^{7}$ who presented the PSD as D50 $-0.7 \mu \mathrm{m}$ and D90 $-2.0 \mu \mathrm{m}$ and a chemical purity of $>99.85 \%$ with a specific surface area of $7 \mathrm{~m}^{2} / \mathrm{g}$. According to the manufacturer the PSD is monomodal. In Table 4.1 the chemical composition of the powder as listed by the manufacturer is given.

Table 4.1 The chemical composition of the Alcoa CT 3000 SG powder as indicated by the manufacturer $^{8}$

\begin{tabular}{cc}
\hline Compound & $\%$ \\
\hline $\mathrm{Al}_{2} \mathrm{O}_{3}$ & 99.8 \\
$\mathrm{Na}_{2} \mathrm{O}$ & 0.08 \\
$\mathrm{Fe}_{2} \mathrm{O}_{3}$ & 0.02 \\
$\mathrm{MgO}$ & 0.07 \\
$\mathrm{SiO}_{2}$ & 0.03 \\
$\mathrm{CaO}$ & 0.02 \\
\hline
\end{tabular}




\subsubsection{Membrane support manufacture before powder pretreatment}

It was initially attempted to manufacture centrifugal casted tubular supports using the Alcoa powder as it has been provided by the supplier. To obtain three tubular ceramic supports with a length of $6 \mathrm{~cm}$, an outer diameter of $20 \mathrm{~mm}$ and a wall thickness of $1.5 \mathrm{~mm}, 42 \mathrm{~g}$ of the powder (Alcoa CT3000 $\mathrm{SG}$ ) was suspended in a mixture of $49 \mathrm{~g}$ distilled water and $10 \mathrm{~g}$ APMA (Ammonium PolyMethAcrylate aqueous solution (25 wt.\%), Darvan C, R.T. Vanderbilt Company, Inc., Norwalk, USA). The same procedure used in the manufacturing of the AKP support membranes as described in Chapter 3 was followed. The following sintering regime was used:

- from room temperature to $400^{\circ} \mathrm{C}$ at a rate of $0.3^{\circ} \mathrm{C} / \mathrm{min}$

- constant heating for 15 minutes at $400^{\circ} \mathrm{C}$ (dispersant burn-off)

- $400^{\circ} \mathrm{C}$ to maximum sintering temperature at a rate of $0.8^{\circ} \mathrm{C} / \mathrm{min}$

- constant sintering for 60 minutes at $1150^{\circ} \mathrm{C}$

- $1150^{\circ} \mathrm{C}$ to room temperature at a rate of $1^{\circ} \mathrm{C} / \mathrm{min}$

A Carbolite CWF1200 (Carbolite, Sheffield, England) oven was used for sintering.

In an attempt to remove impurities from the Alcoa powder and to fractionate the Alcoa powder which had a rather large PSD, two treatments were considered prior to centrifugal casting: an acid treatment and a column treatment.

\subsubsection{Acid treatment of powder}

To remove the majority of fine particles from the Alcoa powder, the powder was dispersed in an acidic aqueous solution. After a period of time the fine particles suspended in the solution were removed. The following procedure was followed:

$600 \mathrm{ml}$ of pure water and a large magnetic stirrer was placed in a $1 \mathrm{~L}$ glass beaker and the $\mathrm{pH}$ was adjusted by adding hydrochloric or nitric acid. After 10 minutes of stirring the $\mathrm{pH}$ was measured and adjusted accordingly. The desired amount of Alcoa CT 3000 SG powder was added and the resulting suspension was stirred for a further hour. The suspension was ultrasonically treated for 10 minutes at a frequency of $20 \mathrm{kHz}$ and a transducer output power of $100 \mathrm{~W}$ (Model 250 Sonifier, Branson Ultrasonics Corporation, Danbury, USA) to break any agglomerates that might have 
formed. The suspension was stirred for a further hour and then left for 48 hours to settle. The liquid at the top of the beaker, which included the fine particles, was carefully removed by means of a pipette and placed into another glass beaker. The amount of liquid removed from the beaker was chosen to avoid disturbing the larger particles which had settled at the bottom of the beaker. Both the larger particles which remained in the $1 \mathrm{~L}$ beaker and the fine particles in the second beaker were dried in an oven overnight at $120^{\circ} \mathrm{C}$. After drying, the various fractions were weighed and then characterized according to size by means of SEM and particle size analysis. A Philips $X L 30$ type SEM was used to obtain images of the various fractions and a Malvern Mastersizer 2000 (Malvern Instrument Ltd., Malvern, UK) instrument was employed for the particle size analysis. A Pump speed of 2000 , maximum allowed obscuration and various amounts of APMA (Ammonium PolyMethAcrylate aqueous solution, Darvan C, R.T. Vanderbilt Company, Inc., Norwalk, USA), as dispersion agent, were used to optimize the particle size analysis. For each analysis $5 \mathrm{~g}$ of powder was dispersed in $5.8 \mathrm{~g}$ of water and a specific amount of polymer. For the acid treatment the following variables were investigated:

- $\mathrm{pH}$ adjusted with hydrochloric or nitric acid $(\mathrm{pH} \mathrm{1.3,1.5,2.0,3.5} \mathrm{and} \mathrm{4)}$

- Amount of starting powder (solid loading: 100, 150 and 200g)

- Amount of APMA used for particle size analysis $(0.005,0.011,0.019,0.022,0.044$ and $0.219 \mathrm{~g} / \mathrm{ml}$ water). $0.219 \mathrm{~g} / \mathrm{ml}$ is the amount used in Chapter 3 for the manufacture of the membranes made from AKP powder.

\subsubsection{Column treatment of powder}

To obtain Alcoa powder fractions with a narrower PSD, a glass column was developed and built to separate various particle sizes making use of gravitational settling. The starting material was the Alcoa powder obtained after the acid treatment (nitric acid) at $\mathrm{pH} 2$ with $200 \mathrm{~g}$ solid loading. Only the larger particles were treated in the column.

Varying amounts of starting powder were separated into 2 portions and placed into 2 separate $500 \mathrm{ml}$ beakers containing wash ethanol ( $3 \mathrm{x}$ the weight amount of powder added) and a large magnetic stirrer. The suspension was stirred for 1 hour and then ultrasonically treated for 15 minutes at a frequency of $20 \mathrm{kHz}$ and a transducer output power of $100 \mathrm{~W}$ (Model 250 Sonifier, Branson Ultrasonics Corporation, Danbury, USA) to break any agglomerates. The two separate 
prepared suspensions were poured into the glass column shown in Figure 4.2 containing $200 \mathrm{ml}$ of wash ethanol.

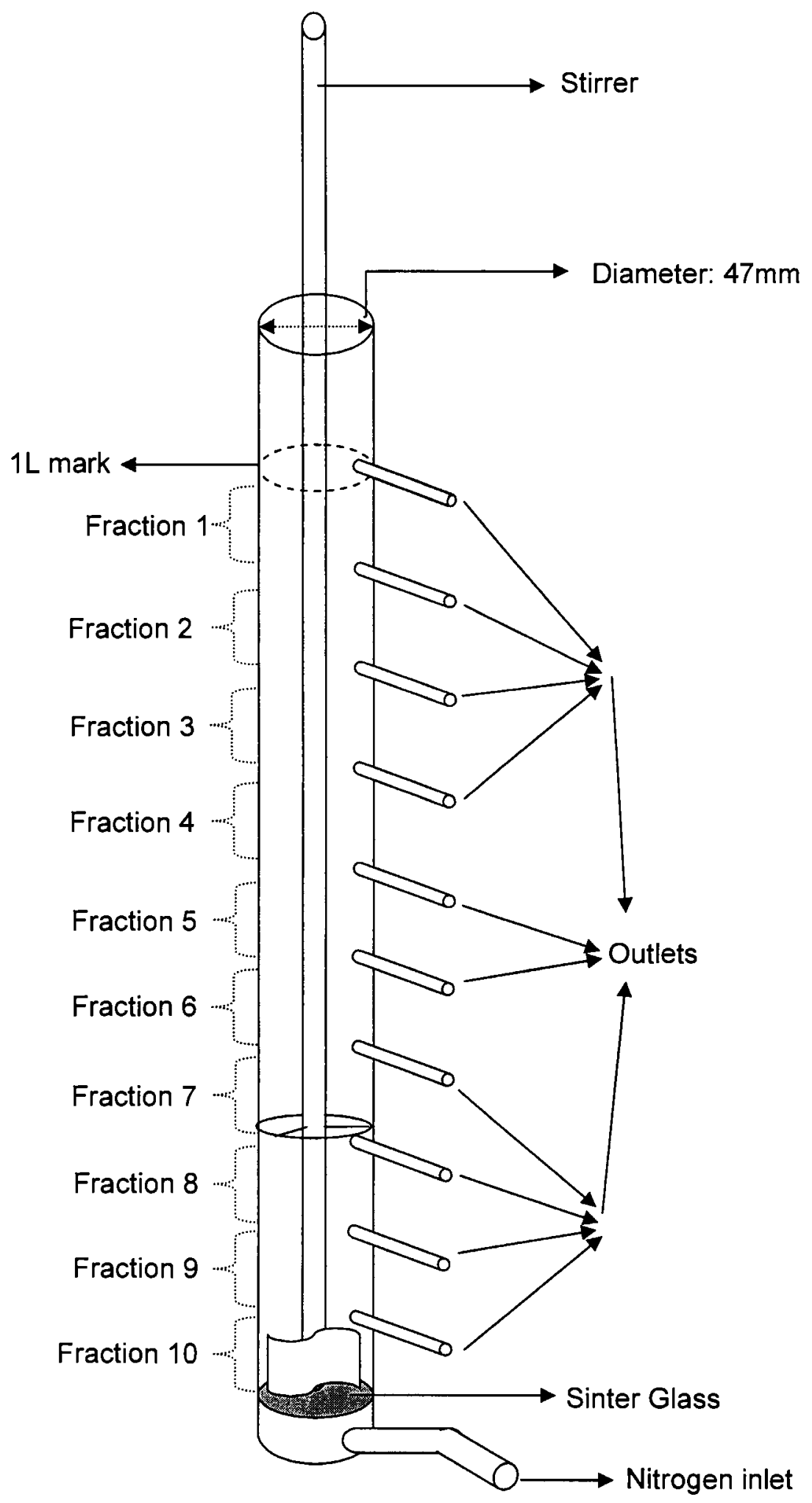

Figure 4.2 Experimental set-up for fractionating column treatment. 
The outlets shown in Figure 4.2 were closed using custom-made taps. The stirrer was then placed into the column, the column filled to the $1 \mathrm{~L}$ mark with wash ethanol and the top sealed with latex material fitted with a rubber pipe, leading to a soap flow meter which was used to measure the nitrogen flow rate. The stirrer was used at a very low stirring rate and the nitrogen pressure was adjusted to obtain the desired flow. The set-up was left for 48 hours at a constant stir and flow rate (bubble rate) whilst adjusting the wash ethanol level at intervals.

After 48 hours the stirring and nitrogen bubbling were stopped and the fractions were removed from the column, starting with fraction 1 . Ten fractions were obtained.

For column treatment the following variables were investigated:

- Powder loading in the column $(100,150$ and $200 \mathrm{~g} / \mathrm{L})$

- Nitrogen flow rate $(1.5,3.0$ and $4.5 \mathrm{ml} / \mathrm{s})$

The fractions obtained were dried in an oven at $80^{\circ} \mathrm{C}$ to remove the wash ethanol and the weight of each fraction was determined. The powder fractions were subsequently characterized using SEM and particle size analysis as described in Section 4.2 .2 . $0.219 \mathrm{~g} / \mathrm{ml}$ APMA was used to disperse the particles in suspension for the particle size analysis.

\subsubsection{Membrane support manufacture after powder pretreatment}

Supports were manufactured using powders from various stages of pretreatment to establish the effect of pretreatment on the PSD of the Alcoa powder. The support preparation was similar to the procedure described in Section 4.2.1. The resulting supports were characterized according to dimensions and by mercury porosimetry, water permeability and SEM. Photographs were taken depending on the quality of the support structure obtained.

\subsubsection{Membranes manufactured after acid pretreatment}

Membranes were manufactured using only the settled (large) particles obtained from the acid treatment described in Section 4.2.2. 


\subsubsection{Membranes manufactured after column pretreatment}

A solid loading of $160 \mathrm{~g}$ of Alcoa powder that had not been acid treated, at a nitrogen flow of $1.5 \mathrm{ml} / \mathrm{s}$, were treated as described in Section 4.2.2. The resulting fractions were dried in an oven to remove the wash ethanol before they were used to manufacture the supports.

\subsubsection{Membranes manufactured after acid and column pretreatment}

The powder used in the manufacturing of these supports was pretreated in the following manner:

- $160 \mathrm{~g}$ of Alcoa powder (CT $3000 \mathrm{SG}$ ) was suspended in $600 \mathrm{ml}$ of water at pH 1.5 (as described in Section 4.2.2) and the fine particles in suspension were removed.

- The heavy fraction was dried in an oven and $140 \mathrm{~g}$ of the powder was then treated with the column fractionation (as described in Section 4.2.3 - nitrogen flow was $1.5 \mathrm{ml} / \mathrm{s}$ ).

- The resulting fractions were placed in an oven to remove the wash ethanol and the dry powder was used to manufacture the supports according to Section 4.2.1. 


\subsection{Results and discussion}

\subsubsection{Membrane supports manufactured before powder pretreatment}

A support manufactured using untreated Alcoa powder is shown in Figure 4.3. Severe cracking and warping of the support structure occurred due to the large PSD of the untreated Alcoa powder ${ }^{3}$, emphasizing the necessity of fractionating the Alcoa powder in order to obtain a defect free $\alpha$-alumina support structure by means of centrifugal casting.

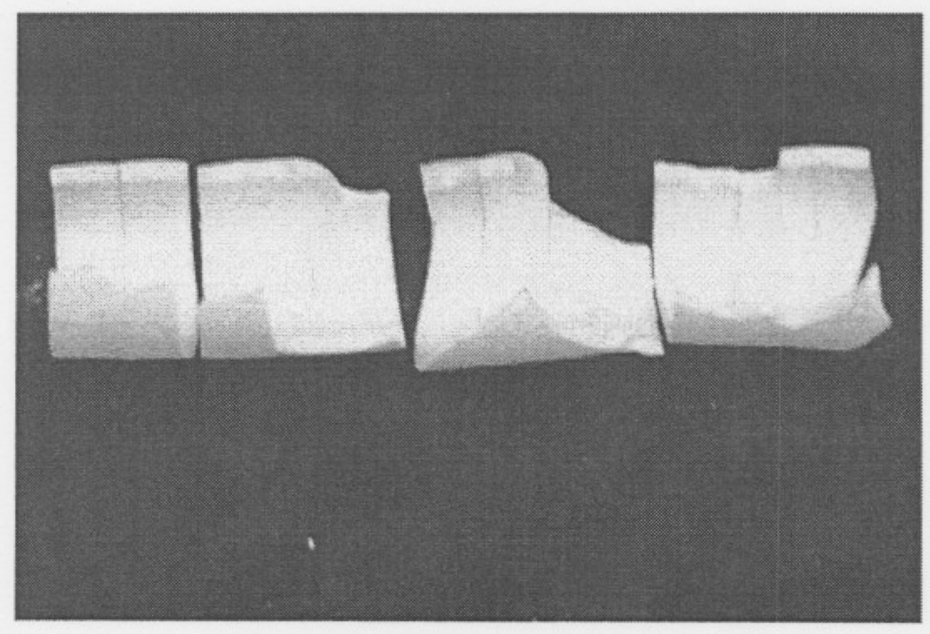

Figure 4.3 A support manufactured without any pretreatment of the Alcoa powder.

The severe cracking and warping of the support illustrated in Figure 4.3 seemed to suggest that the particle size distribution of the Alcoa powder was too large to manufacture centrifugal casted membrane supports. In an attempt to reduce the size distribution and to remove the impurities present in the Alcoa powder, an acid treatment and a column treatment were performed.

\subsubsection{Acid treatment of powder}

According to Table 4.2, there was an increase in the amount of fine particles remaining in suspension with an increasing amount of solid loading for the $\mathrm{pH} 1.3, \mathrm{pH} 1.5$ and $\mathrm{pH} 2$ treatments, 
irrespective of the type of acid used. For the higher $\mathrm{pH}$ treatment, namely at $\mathrm{pH} 3$ and $\mathrm{pH} \mathrm{4.5,} \mathrm{a}$ negligible amount of fine particles were still suspended in the water after 48 hours. When using $\mathrm{HNO}_{3}$ instead of $\mathrm{HCl}$, the $\mathrm{pH}$ of the solution had a larger effect on the concentration of fine particles in suspension than the solid loading. The highest concentration of fine particles in the suspension at $\mathrm{pH} 1.5$ could not be explained. Both $\mathrm{HCl}$ and $\mathrm{HNO}_{3}$ are strong acids (their acid ionization constant $K_{\mathrm{a}}$ is large) and both dissociate completely in water. Thus, it would be expected that similar tendencies would be obtained for both $\mathrm{HCl}$ and $\mathrm{HNO}_{3}$. This is confirmed by the average amount suspended which was in the order of $22 \% \mathrm{~m} / \mathrm{m}$ for $\mathrm{HCl}$ and $20 \% \mathrm{~m} / \mathrm{m}$ for the $\mathrm{HNO}_{3}$ in the $\mathrm{pH}$ range 1.3 to 2 .

Table 4.2 Concentration of fine particles in suspension after 48 hours at various pH values in the presence of $\mathrm{HCl}$ or $\mathrm{HNO}_{3}$

\begin{tabular}{|c|c|c|c|c|c|c|}
\hline \multirow{4}{*}{ pH set } & \multicolumn{6}{|c|}{$\begin{array}{l}\text { Concentration of fine particles in suspension }(\% \mathrm{~m} / \mathrm{m}) \\
\qquad(\mathrm{m} / \mathrm{m}=\text { mass in suspension } / \text { mass of loading })\end{array}$} \\
\hline & \multicolumn{3}{|c|}{$\mathrm{HCl}$} & \multicolumn{3}{|c|}{$\mathrm{HNO}_{3}$} \\
\hline & \multicolumn{3}{|c|}{ Solid loading $(g)$} & \multicolumn{3}{|c|}{ Solid loading (g) } \\
\hline & 100 & 150 & 200 & 100 & 150 & 200 \\
\hline 1.3 & 16 & 17 & 33 & 10 & 14 & 13 \\
\hline 1.5 & 19 & 20 & 27 & 30 & 27 & 30 \\
\hline 2.0 & 16 & 26 & 28 & 16 & 21 & 20 \\
\hline 3.0 & 0 & 0 & 0 & 0 & 0 & 0 \\
\hline 4.5 & 0 & 0 & 0 & 0 & 0 & 0 \\
\hline
\end{tabular}

When using $\mathrm{HCl}$, the variation in $\mathrm{pH}$ did not significantly influence the amount of alumina present in suspension. In the case where the $\mathrm{pH}$ remained constant and the solid loading was altered, an increase in solubilization with increasing loading was observed for $\mathrm{HCl}$. According to HofmanZuter, ${ }^{9}$ the $\mathrm{pH}$ can strongly influence reactions of $\alpha$-alumina. The author suggests that alumina hydrates in water, forming two different products:
(a) $\mathrm{Al}_{2} \mathrm{O}_{3}+3 \mathrm{H}_{2} \mathrm{O} \rightarrow 2 \mathrm{Al}(\mathrm{OH})_{3}$
and
(b) $\mathrm{Al}_{2} \mathrm{O}_{3}+\mathrm{H}_{2} \mathrm{O} \rightarrow 2 \mathrm{AlOOH}$ 
After the hydration, hydrogen ion adsorption occurs:
(a) $2 \mathrm{Al}(\mathrm{OH})_{3}+\mathrm{H}^{+} \rightarrow 2 \mathrm{Al}(\mathrm{OH})_{2}{ }^{+}+\mathrm{H}_{2} \mathrm{O}$
and
(b) $\mathrm{AlOOH}+\mathrm{H}^{+} \rightarrow \mathrm{Al}(\mathrm{OH})_{2}{ }^{+}$

resulting in the formation of the following products:

$\mathrm{Al}(\mathrm{OH})_{2}{ }^{+}+2 \mathrm{H}^{+} \rightarrow \mathrm{Al}^{3+}+2 \mathrm{H}_{2} \mathrm{O}$

$\mathrm{Al}(\mathrm{OH})_{2}{ }^{+}+\mathrm{Cl}^{-} \rightarrow \mathrm{Al}(\mathrm{OH})_{2} \mathrm{Cl}$

$\mathrm{Al}(\mathrm{OH})_{2}{ }^{+} \rightarrow \mathrm{AlOOH}+\mathrm{H}^{+}$

The amount of alumina that settles in an acidic solution would thus be influenced by the amount of alumina that reacts with the hydrochloric acid to form the products shown. It is therefore possible that, if more alumina is present, a smaller portion of the alumina would form the products mentioned and, consequently, a larger amount of alumina would remain present in the suspension, explaining why the $200 \mathrm{~g}$ loading resulted in a larger amount of the alumina particles present in the suspension after 48 hours.

Another possible explanation could be related to the settling of the charged alumina particles (below pH $8 \alpha$-alumina particles are positively charged ${ }^{10}$ ). During the settling of the positively charged particles, a positively charged layer is formed at the bottom of the beaker. The higher solid loading created a volumetric limitation on the amount of alumina that was able to settle due to the positively charged layer at the bottom of the beaker (repulsive forces between two similarly charged entities) and consequently a larger amount of alumina was found in suspension after 48 hours.

According to the results, a reasonable amount of particles remained in suspension after 48 hours for both the $\mathrm{HCl}$ and $\mathrm{HNO}_{3}$ in the $\mathrm{pH}$ range of 1.3 to 2, while the rest of the particles had settled. Singh et al., ${ }^{11}$ who also studied the dispersion of a-alumina Alcoa CT-3000 SG powder at various $\mathrm{pH}$ values for a period of 48 hours, set the $\mathrm{pH}$ using $\mathrm{HCl}$ and $\mathrm{NaOH}$ and obtained stable suspensions between $\mathrm{pH} 4$ and $\mathrm{pH} 7$ which is in contrast to this study. The difference could be due to the fact that Singh et al. ${ }^{11}$ used very low solid loading concentrations $(0.05 \mathrm{~g} / \mathrm{ml})$ compared to this study where the solid loading was between 0.17 and $0.33 \mathrm{~g} / \mathrm{ml}$. A higher solid loading leads to the formation of agglomerates resulting in a quicker sedimentation of the powder and a less stable suspension. According to the specific surface charge obtained by Singh et al., ${ }^{11}$ the most stable suspension would be at $\approx \mathrm{pH}$. The good dispersion quality of the alumina suspension at low $\mathrm{pH}$ values is due to a high positive surface charge in an acidic environment, which results from alumina being a basic oxide which consumes $\mathrm{H}^{+}$, resulting in a positively charged particle and consequentially a well dispersed suspension. Due to the higher solid loading in this study, a higher 
amount of positive charges would be required to obtain a stable dispersion for a longer period of time.

The SEM images of the (a) untreated Alcoa powder, (b) fine fraction (the particles in suspension) and the (c) larger fraction (sediment) are shown in Figure 4.4. Similar images were obtained for all the $\mathrm{pH}$ ranges and solid loadings for both the $\mathrm{HCl}$ and $\mathrm{HNO}_{3}$ treatments. It is clearly visible that there were both fine and large particles present in the untreated sample (Figure 4.4a). In Figure $4.4 \mathrm{~b}$ it can be seen that most of the suspended particles were of similar size and smaller than $1 \mu \mathrm{m}$ compared to the sediment (Figure 4.4c). Many of the particles in the sediment (Figure 4.4c) were significantly larger and a wider range in sizes was observed. Both large and small particles were still present in the settled fractions, irrespective of the treatment condition. This observation confirms that the larger particles settle due to gravitational force, while the fine particles remained in suspension for a longer period of time due to the repulsive forces of the charged finer particles.

The particle size analysis for the fine and heavy fractions of the powder treatment at $\mathrm{pH} 2$ with $\mathrm{HCl}$ and the $200 \mathrm{~g} / \mathrm{L}$ solid loading as well as the PSD of the untreated Alcoa powder is shown in Figure 4.5. The amount of polymer used for dispersion was $0.219 \mathrm{~g} / \mathrm{ml}$.

The difference between the fine and heavy fractions are clearly visible. The vol. $\%$ of small particles (the peak at $0.17 \mu \mathrm{m}$ ) in the heavy fraction was much less than in the fine fraction ( 4 vol. $\%$ vs. 10 vol. \%). The vol. $\%$ of larger particles in the fine fraction (the peak at $0.5 \mu \mathrm{m}$ ) was only slightly visible while the larger particles in the heavy fraction were at a larger particle size $(0.8 \mu \mathrm{m})$ and had a greater intensity (4 vol. \%). The vol. \% large particles in the heavy fraction (the peak at $0.8 \mu \mathrm{m}$ ) were more than the vol. \% present in the untreated Alcoa powder. The particle size analysis clearly illustrates the fact that the finer particles were removed during the acid treatment step of the Alcoa powder and confirms the observations made from the SEM images. The fine fraction (suspended particles) consisted of larger vol. \% of small particles, while there was an equal vol. \% of small and large particles present in the heavy fraction. No noticeable difference could be observed for the PSD of the fine and the heavy fractions between the $\mathrm{HCl}$ and the $\mathrm{HNO}_{3}$ treatments. 


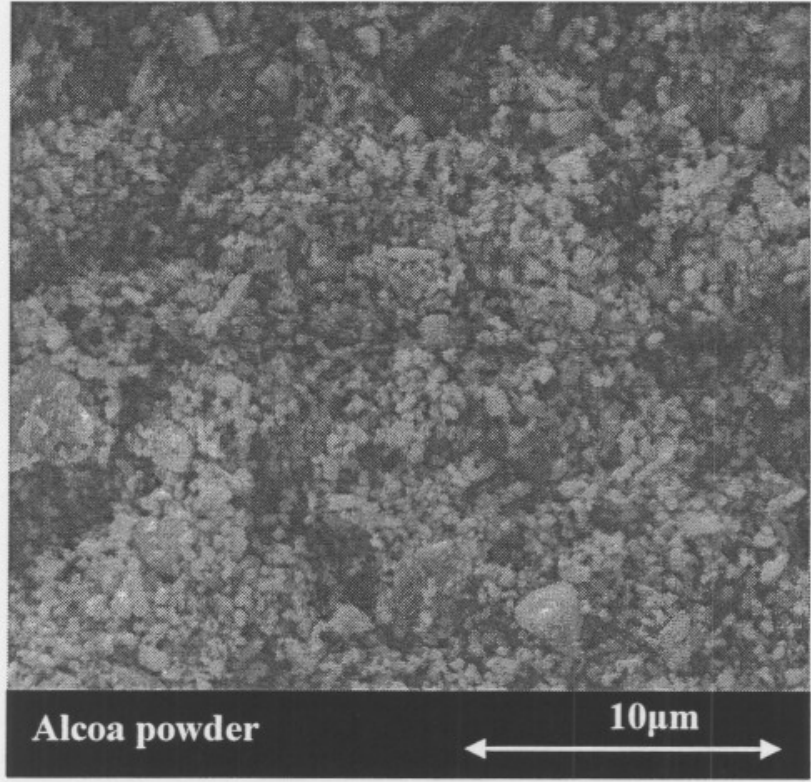

(a)

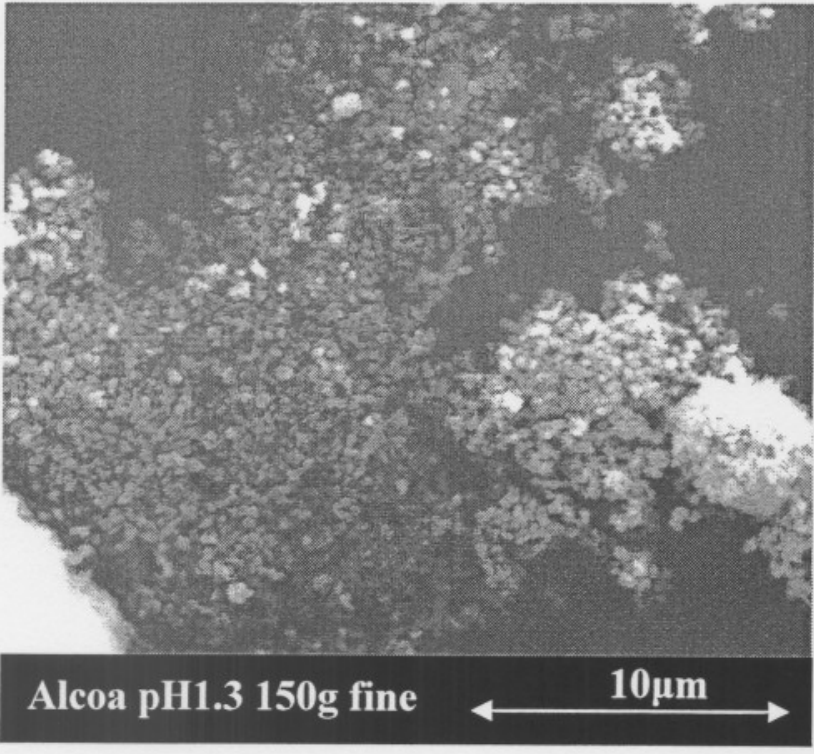

(b)

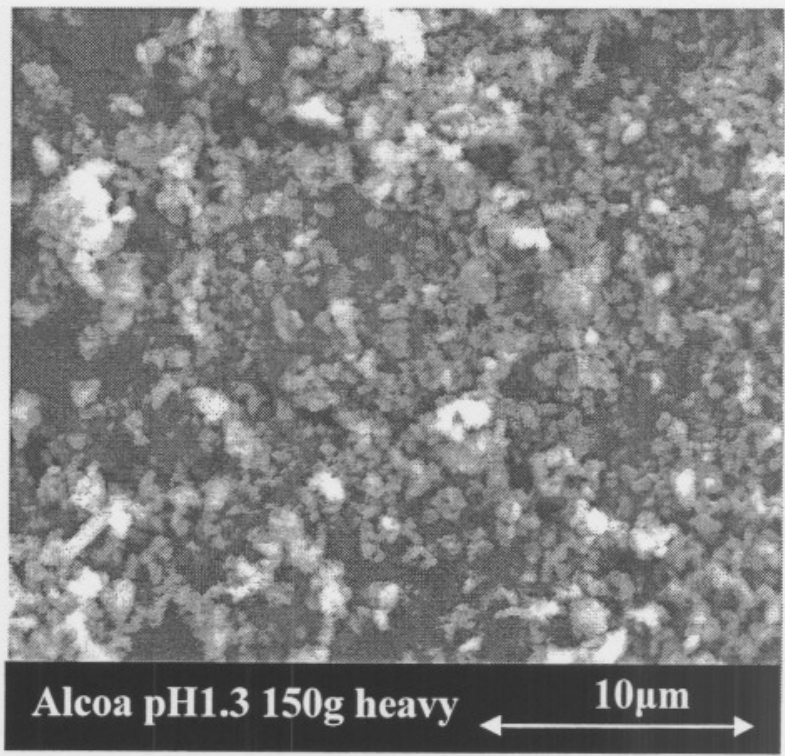

(c)

Figure 4.4 (a) Untreated Alcoa powder, (b) suspended and (c) settled Alcoa CT 3000 SG powder after 48 hours. 


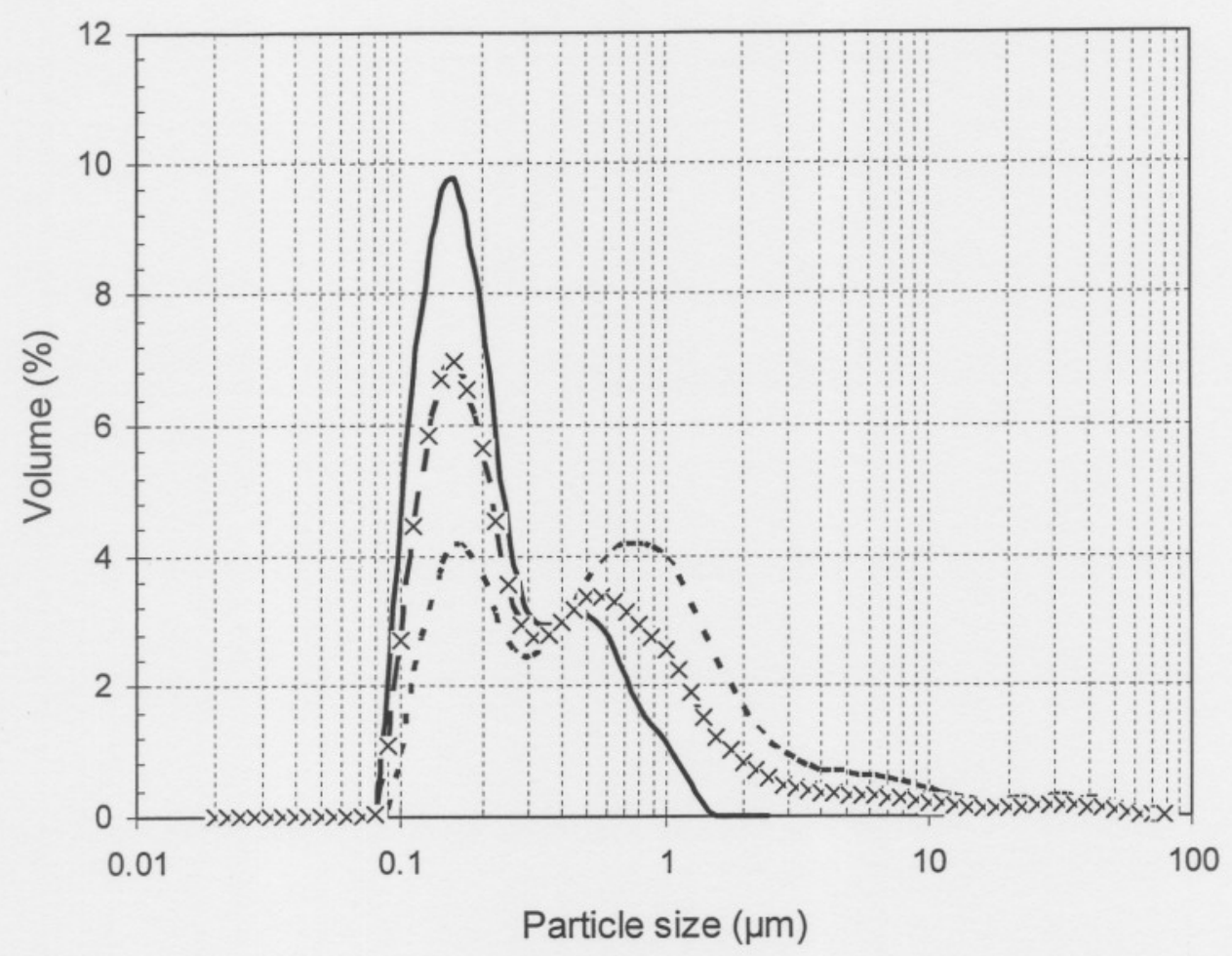

Figure 4.5 PSD of the fine and heavy fraction for the $\mathrm{pH} 2$ treatment and a $200 \mathrm{~g} / \mathrm{L}$ solid loading.

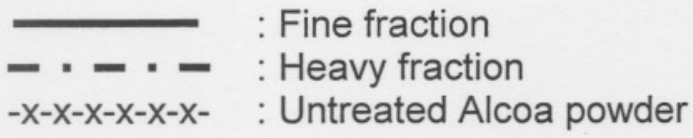

It seems, however, that the amount of polymer used for the preparation of the suspension for the PSD analysis had an significant influence on the analysis of the fine fraction $(\mathrm{pH} 2$, solid loading of $200 \mathrm{~g} / \mathrm{L}$ ) as illustrated in Figure 4.6. In the concentration range $0.011 \mathrm{~g} / \mathrm{ml}$ to $0.219 \mathrm{~g} / \mathrm{ml}$, similar distributions were obtained, which correlated well with the fine fraction observed in Figure 4.5. In Figure 4.6 only the $0.022 \mathrm{~g} / \mathrm{ml}$ and the $0.219 \mathrm{~g} / \mathrm{ml}$ concentrations are shown as the $0.011,0.019$ and $0.049 \mathrm{~g} / \mathrm{ml}$ distributions were very similar. Above $0.011 \mathrm{~g} / \mathrm{ml}$ a bimodal distribution was obtained. At a polymer concentration of $0.005 \mathrm{~g} / \mathrm{ml}$ a single peak at approximately $2.8 \mu \mathrm{m}$ was observed, which suggests that agglomeration occurred during the addition of the suspension to the water reservoir of the Malvern Mastersizer. According to the datasheet of the CT 3000 SG provided by Alcoa and Studard et al. ${ }^{7}$, the powder is monomodal. However, in view of this study it seems that the CT 3000 SG powder is only monomodal if inadequate or no polymer is added prior to the PSD analysis. It is clearly shown that when enough polymer is added to disperse all agglomerates, the PSD of Alcoa CT 3000 SG actually has a bimodal distribution. 


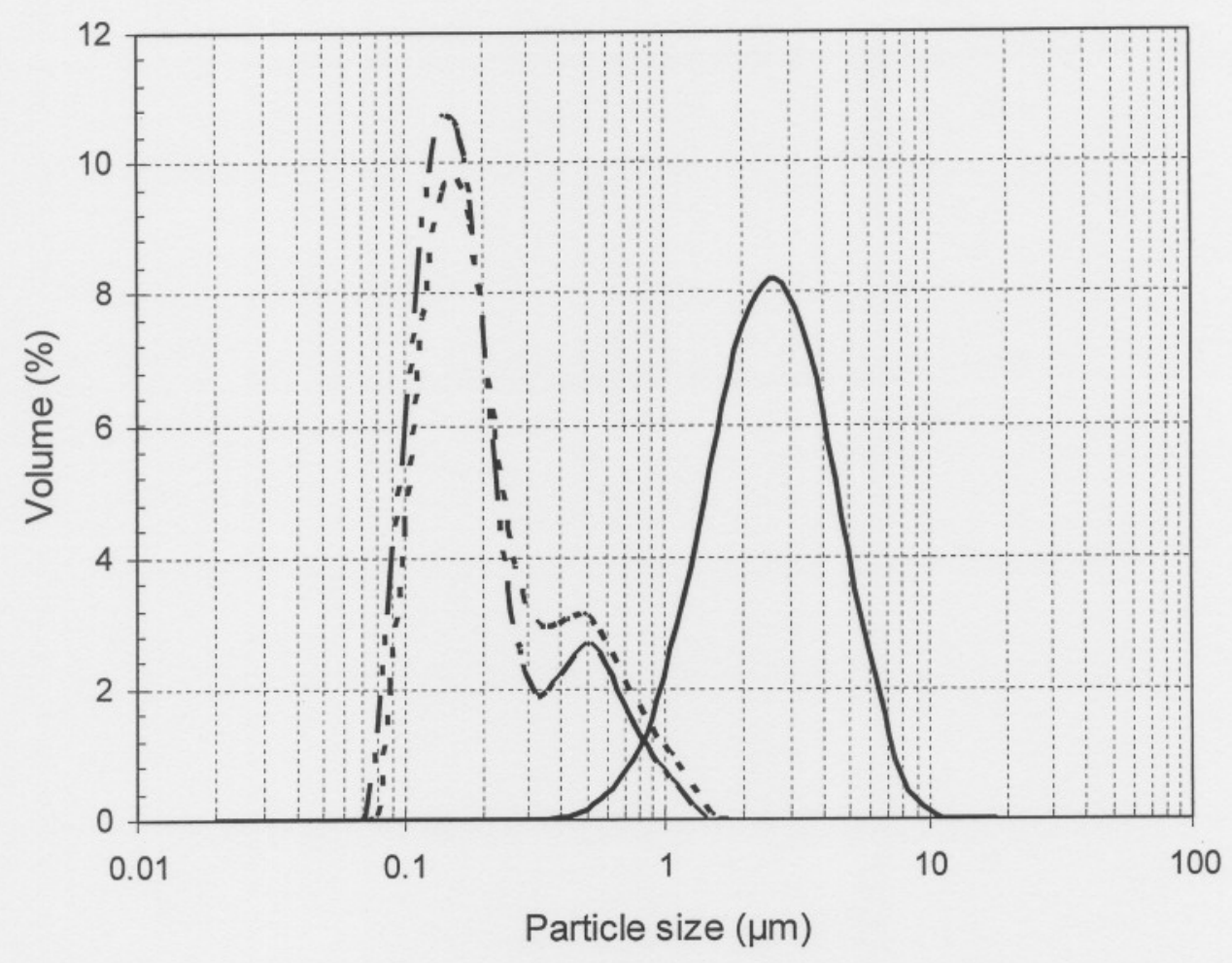

Figure 4.6 Influence of the amount of polymer added to a suspension of fine Alcoa powder ( $\mathrm{pH} 2,200 \mathrm{~g} / \mathrm{L}$ solid loading) for PSD analysis.

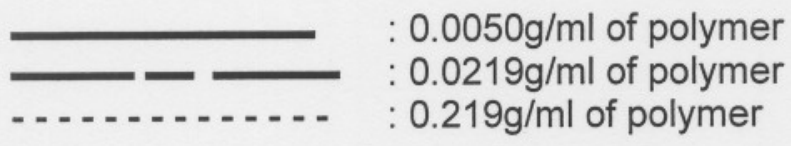

From the data obtained from the acid treatment study, it is clear that fine particles remained in suspension. This method is therefore suitable for decreasing the large percentage of fine particles present in the Alcoa powder, but not appropriate for complete fractionation as some large particles were also removed while a large amount of fine particles remained in the heavy fraction.

\subsubsection{Column treatment of powder}

After the acid treatment $\left(\mathrm{HNO}_{3}, \mathrm{pH} 2\right.$ and $200 \mathrm{~g} / \mathrm{L}$ solid loading) the heavy fraction of the Alcoa powder was further fractionized using the fractionizing column.

According to Table 4.3, the weight of powder in each fraction varied considerably for each column treatment irrespective of the loading. The amount of powder in each fraction (constant powder loading) remained constant with variation in nitrogen flow rate. The solid loading did, however, 
influence the amount in each fraction. In Table 4.3 the mass of powder present in each fraction with a change in solid loading of the column is listed.

Table 4.3 Mass of powder present in each fraction for the three solid loadings evaluated

\begin{tabular}{|c|c|c|c|}
\hline \multirow[t]{3}{*}{ Fraction } & \multicolumn{3}{|c|}{ Mass of powder in each fraction $(\mathrm{g})$} \\
\hline & & Solid loading $(g / L)$ & \\
\hline & 100 & 150 & 200 \\
\hline 1 & $6.5 \pm 1$ & $11 \pm 1$ & $15 \pm 1.5$ \\
\hline \multirow[t]{2}{*}{$2-8$} & $72 \pm 7$ & $108 \pm 9$ & $144 \pm 11.5$ \\
\hline & (9g each) & (13.5g each) & (18g each) \\
\hline 10 & $21.5 \pm 1.5$ & $31 \pm 2$ & $41 \pm 4.5$ \\
\hline
\end{tabular}

From Table 4.3 it is obvious that the amount of powder in each fraction increased with increasing solid loading as would be expected due to the larger amount of powder present in the column. The amount of powder present in fraction 10 was the highest while it was the lowest in fraction 1 which is due to gravity resulting in the highest particle packing density at the bottom of the column.

According to the PSD analysis shown in Figure 4.7, no statistically significant fractionation was obtained by means of the column for the $150 \mathrm{~g} / \mathrm{L}$ solid loading. The PSD of the fractions varied insignificantly and although a slight trend was observed, the variation in vol. \% was high $\left(R^{2}=0.19\right.$ for the straight line of the larger particles). For the larger particles, there was an overall increase in vol. \% with increasing fraction number while there was a decrease in vol. \% with increasing fraction number. In Figure 4.8, the PSD of fractions 4,5 and 6 are shown. When these results are compared to the starting powder PSD (heavy fraction of Figure 4.5), a slight difference is noticeable. In the PSD of the column treatment, the peak at $\approx 0.17 \mu \mathrm{m}$ has slightly increased in intensity, suggesting that a larger fraction of fine particles was present in the fractions which had been obtained by the column fractionation. It would then be expected that in one of the PSD's (possibly fraction 10 ) there would be an increase in the peak intensity at $\approx 0.8 \mu \mathrm{m}$ due to the fact that there was an increase in fine particle sizes in the other fractions. This was, however, not observed and it should be concluded that the Malvern Mastersizer analysis is not suitable to measure such minute differences in particle sizes accurately. Similar results for the 100 and $200 \mathrm{~g} / \mathrm{L}$ solid loading and the variation in nitrogen flow rate were observed. 


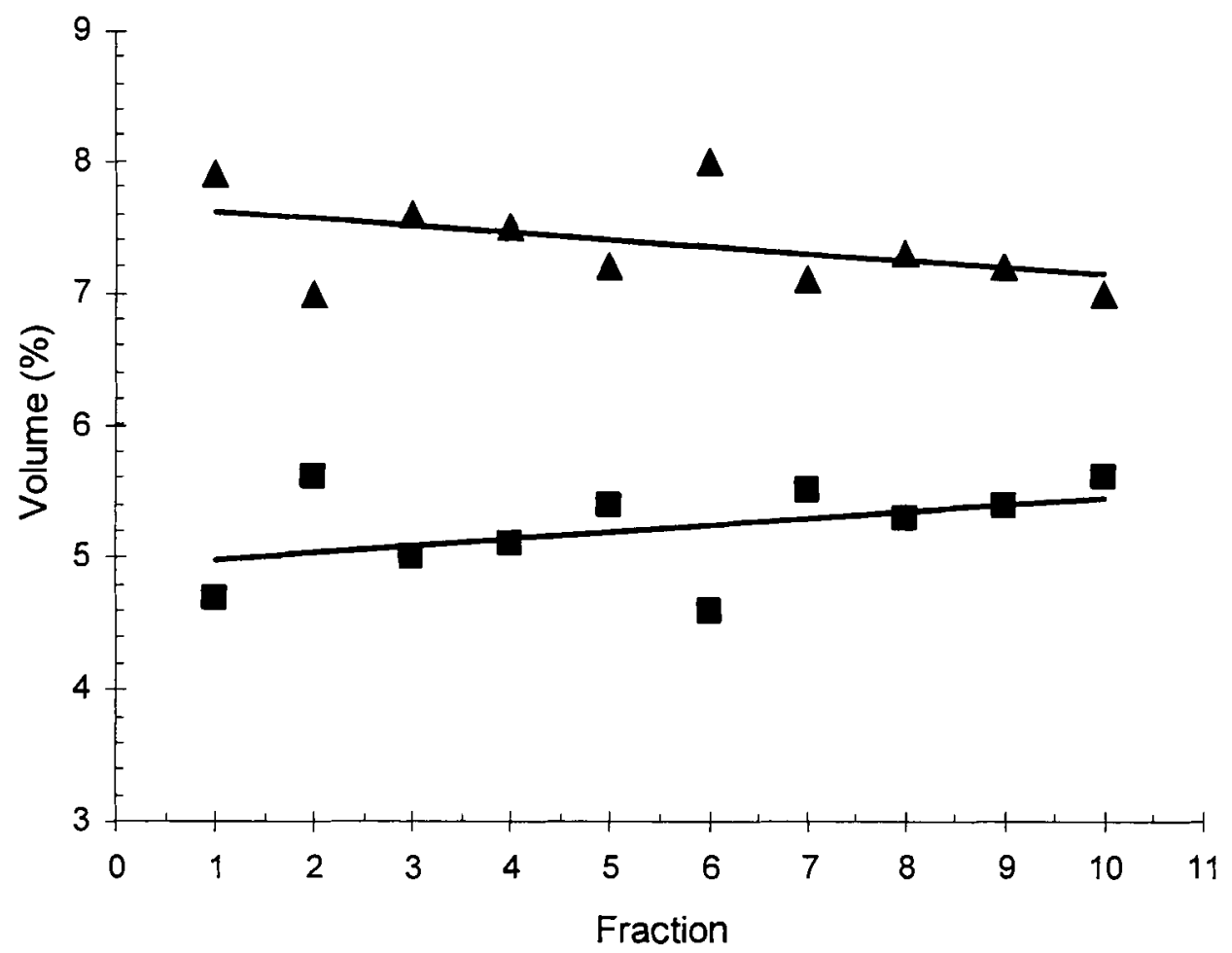

Figure $4.7 \quad$ Volume $\%$ of the heavy and fine particles as a function of fractions for the $150 \mathrm{~g} / \mathrm{L}$ solid loading with a nitrogen flow rate of $1.5 \mathrm{ml} / \mathrm{s}$
(A) : Fine particles
(a) : Heavy particles 


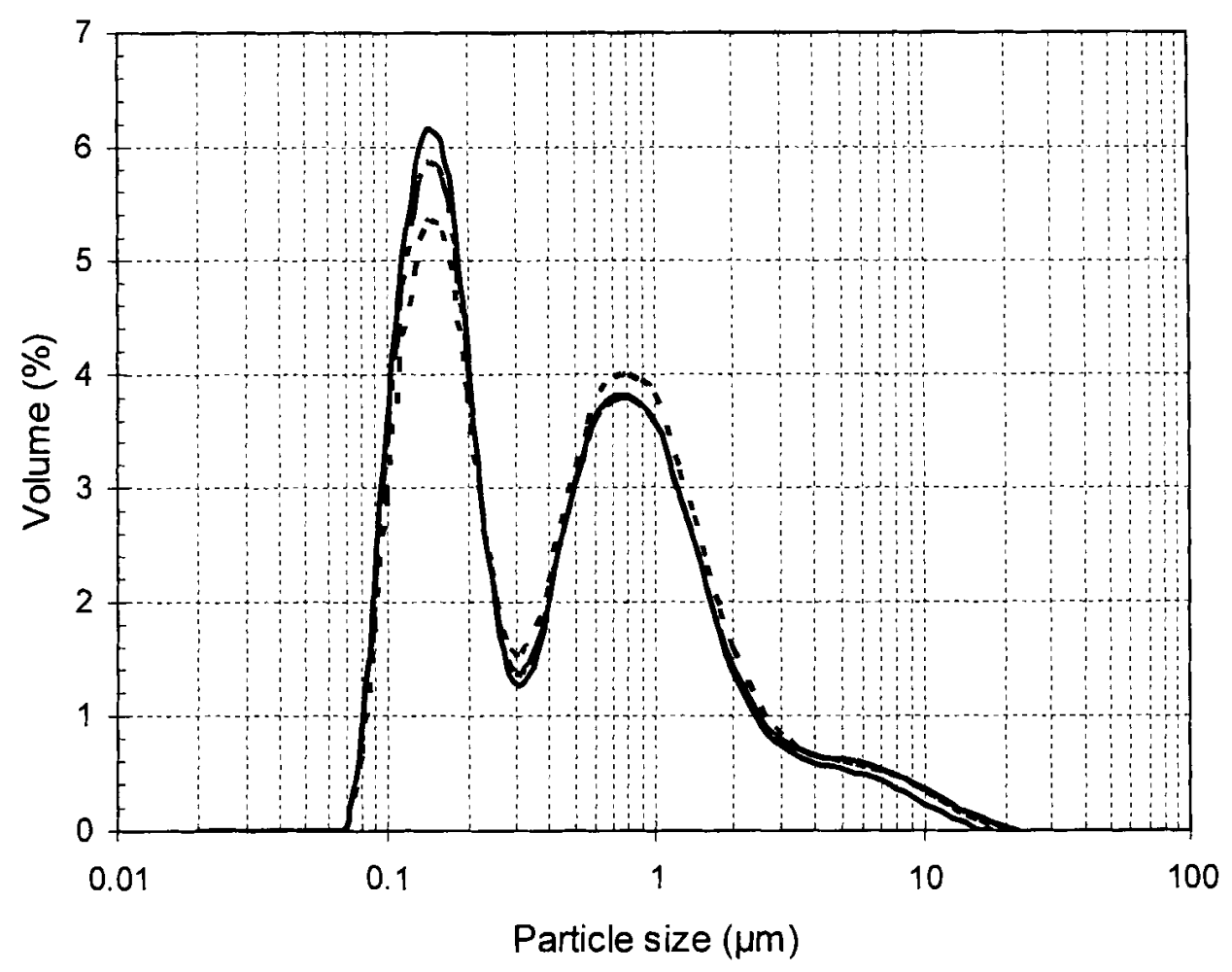

Figure 4.8 PSD of the various fractions during the column treatment.

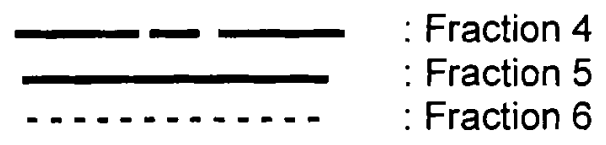

\subsubsection{Membrane support manufacture after powder pretreatment}

The powder fractions obtained from the pretreatment (the acid treatment and/or column treatment) were used to manufacture membranes. The supports manufactured using only the acid treatment or only the column treatment resulted in tubular supports with defective inner surfaces. However, the supports manufactured by combining the acid and column treatments (acid treatment first) resulted in supports with smooth inner surfaces and defect free outer structures. Although the attempts mentioned resulted in successful support structures initially, all further attempts to repeat the manufacture of the defect-free membranes failed, emphasizing the low repeatability of the fractionation procedures employed. 


\subsubsection{Membranes manufactured after acid pretreatment}

The supports manufactured using powder pretreated only with the acid treatment procedure was defective and could only be characterized by SEM.

Figure 4.9 displays the (a) large and (b) small magnification SEM images of an Alcoa support surface where only the fine particles were removed (acid treatment at $\mathrm{pH}=2$ with $\mathrm{HCl}$ and $200 \mathrm{~g}$ solid loading as described in Section 4.2.2). No column treatment was performed. It is clearly visible that the particle packing was not homogeneous and a large variation of particle sizes was present (Figure 4.9a). With the low magnification the severe surface cracking is visible (Figure $4.9 b)$. The surface cracking can be attributed to the wide PSD still present in the powder, which results in differences in sintering rates in various regions of the green cast during sintering and hence the formation of cracks. ${ }^{3}$

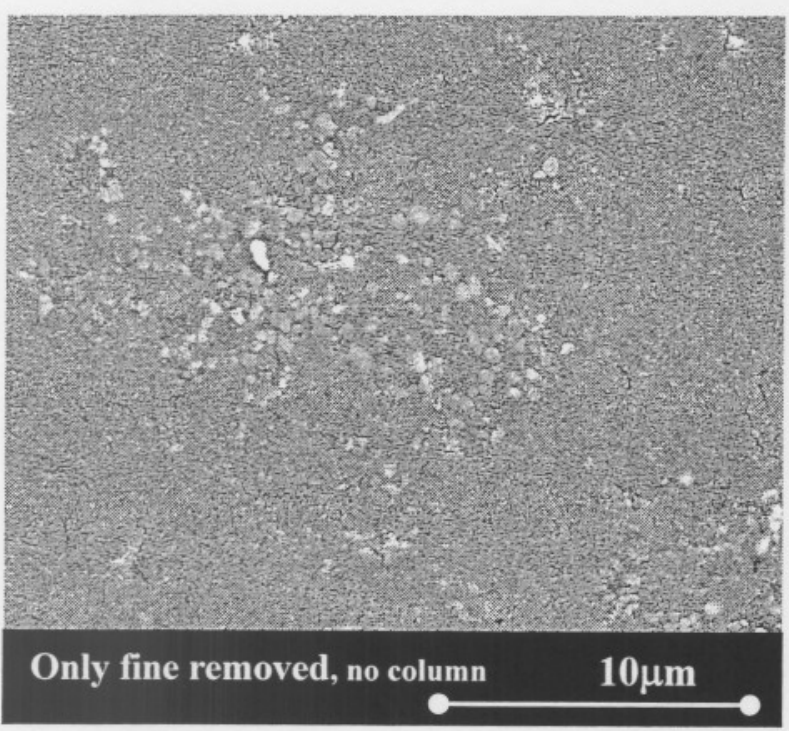

(a)

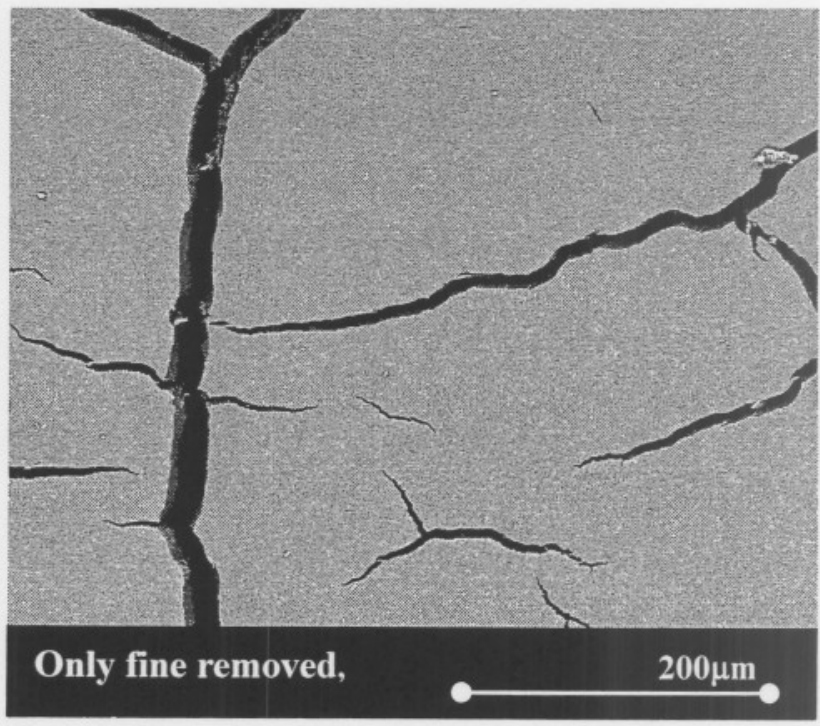

(b)

Figure 4.9 The (a) high and (b) low magnification of the Alcoa support where only fine particles were removed by an acid treatment. 


\subsubsection{Membranes manufactured after column pretreatment}

The supports manufactured using Alcoa powder pretreated with only the column treatment resulted in membranes with slight surface defects on the inside, but a defect free outer support structure. Only the following 3 combinations of fractions were used to manufacture three membranes:

$>$ Fractions 4 and 5 (Alcoa1)

Fractions 6 and 7 (A/coa2)

$>$ Fractions 8 and $9(A /$ coa3 $)$

The three membranes produced were characterized by mercury porosimetry, water permeability and SEM. The supports were also photographed.

\subsection{Mercury porosimetry}

The porosity and the median pore diameter were affected by the fractions used as can be seen from Table 4.4. The fractions higher up in the column (lower numbered fractions) resulted in a support structure with a lower porosity and a smaller pore diameter. Kinery et al. ${ }^{12}$ indicated that the smaller the initial powder size, the larger the densification and grain growth rates during sintering. The increased porosity and median pore diameter with increasing fraction number (Alcoa1 - Alcoa3) confirms the difference in starting powder of the various membranes and the fact that fractionation occurred during the column treatment. ${ }^{14}$

Table 4.4 The porosity, median pore diameter and water permeability of the Alcoa1-Alcoa3 supports. The supports were manufactured with powder pretreated with only the column treatment and had defects on the inside surface.

\begin{tabular}{cccc}
\hline Support & $\begin{array}{c}\text { Median pore diameter } \\
(\mathrm{nm})\end{array}$ & $\begin{array}{c}\text { Porosity } \\
(\%)\end{array}$ & $\begin{array}{c}\text { Water permeability } \\
\left(\mathrm{L} . \mathrm{m}^{2} \cdot \mathrm{h}^{-1} \cdot \text { bar }^{-1}\right)\end{array}$ \\
\hline Alcoa1 & 114.8 & 38.7 & 28.8 \\
Alcoa2 & 115.6 & 39.0 & 30.4 \\
Alcoa3 & 118.6 & 39.8 & 30.8 \\
\hline
\end{tabular}


The pore size distribution of all the fractions was monomodal, as illustrated in Figure 4.10. The Alcoa1 fraction had the narrowest distribution and the smallest median pore size while, the Alcoa3 fraction had the widest distribution. The change in the pore size distribution suggests that there was a difference in the particle size distribution of the various fractions since a narrow particle size distribution results in a narrow pore size distribution, while a wide particle size distribution would result in a wider pore size distribution. ${ }^{4}$ According to these observations, the fractions situated at the top of the column during the column treatment (lower numbered fractions), consisted of a narrower particle size distribution than the fractions at the bottom (higher numbered fractions) which could be explained in terms of the gravitational settling. The small particles settle at a slower rate than the larger particles and consequently are found higher up in the column. However, due to the agglomeration of large and small particles, the fractions at the bottom have a larger particle size distribution than the fractions at the top.

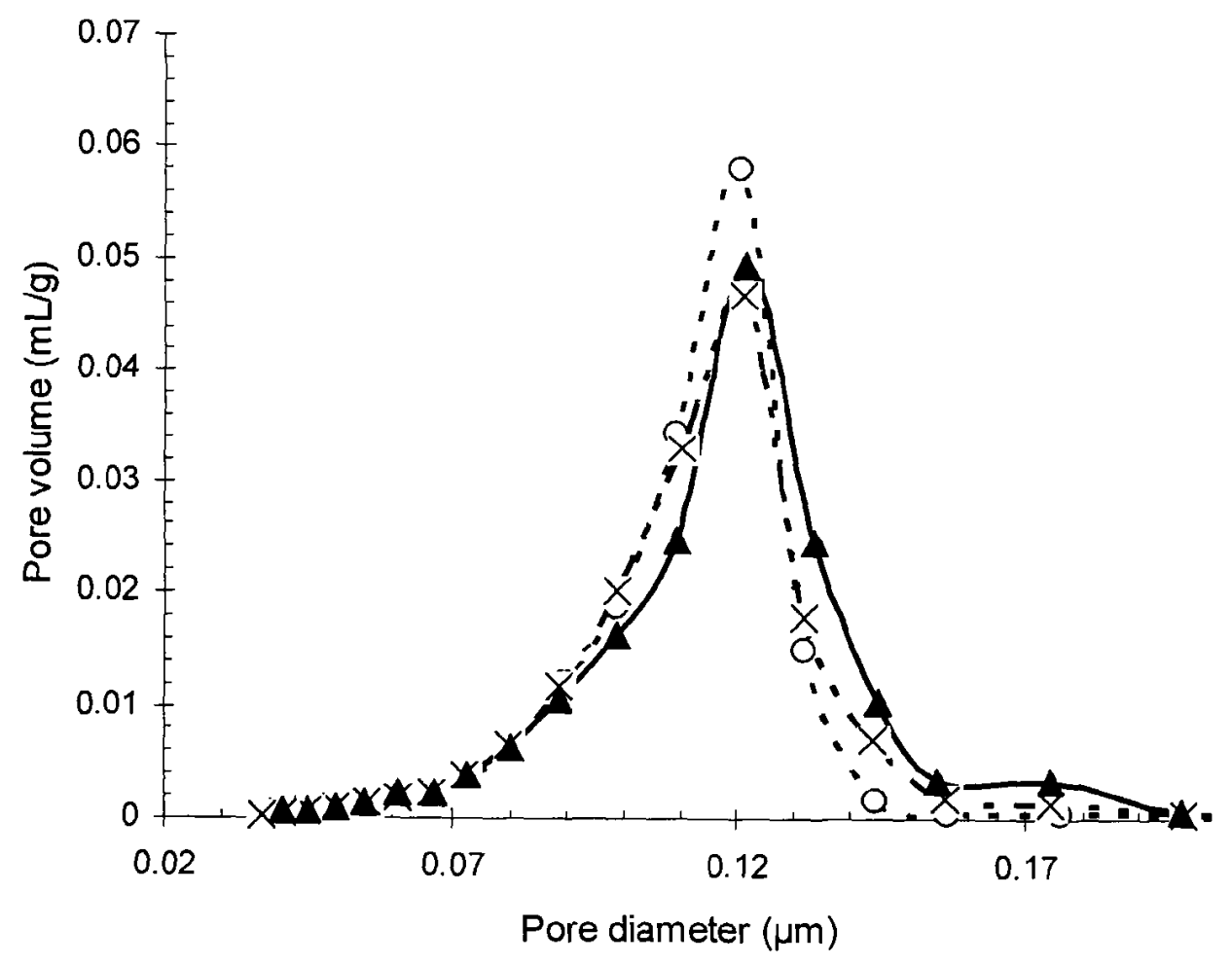

Figure 4.10 Pore volume as a function of pore diameter for the Alcoa1, Alcoa2 and Alcoa3 supports sintered at $1150^{\circ} \mathrm{C}$.

$-0-1$ Alcoa1
$-x-1$ Alcoa2
$-A-:$ Alcoa3 


\subsection{Water permeability}

The pore size and the porosity influenced the water permeability as seen in Table 4.4, which is in agreement with the Hagen-Poiseule equation. ${ }^{13}$ The larger pore size and porosity of the Alcoa3 compared to the A/coa1 and Alcoa2 supports resulted in an increased water permeability.

\subsection{Photograph}

A photograph of a support produced in a similar manner as the Alcoa1 - Alcoa3 is shown in Figure 4.11. The support had a defect free outer structure but flawed inner surface that cracked and tore from the outer structure during sintering. The tearing and cracking is due to the difference in sintering rates between the large particles on the outside and the small particles on the inside of the tubular structure. ${ }^{3}$

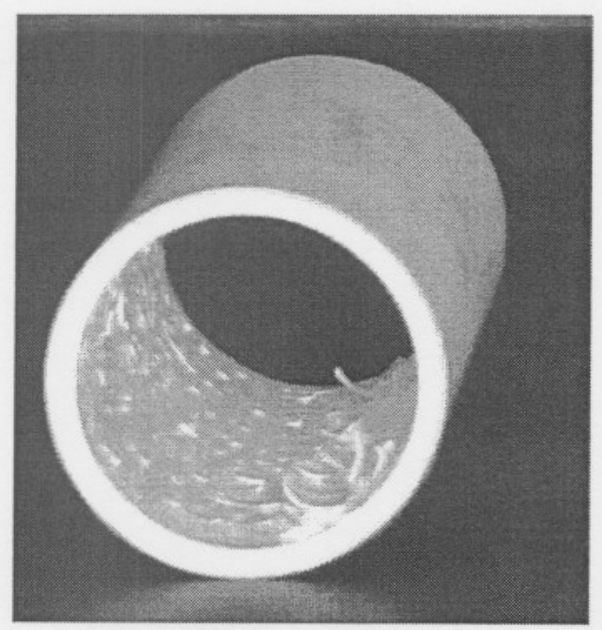

Figure 4.11 a Photograph of a support manufactured with Alcoa powder pretreated with only the column treatment.

\subsubsection{Membranes manufactured after acid and column pretreatment.}

The supports manufactured using Alcoa powder pretreated with both the acid treatment and the column treatment resulted in membranes with smooth inner surfaces and a defect free outer 
support structure. The following 3 combinations of fractions were used to manufacture these membranes:

$>$ Fractions 4 and 5 (Alcoa4)

$>$ Fractions 6 and 7 (Alcoa5)

$>$ Fractions 8 and $9($ Alcoa6)

The three $\alpha$-alumina membrane supports without defects (Alcoa4, Alcoa5 and Alcoa6) were characterized according to dimensions and by mercury porosimetry, water permeability and SEM. The supports were also photographed.

\subsection{Support dimensions and linear shrinkage}

Table 4.5 lists the support dimensions and linear shrinkage during sintering at $1150^{\circ} \mathrm{C}$. There was an increase in outer diameter and a corresponding decrease in linear shrinkage for the fractions situated closer to the bottom of the column. Since the heavier (larger) particles are predominantly found at the bottom part of the column (gravity), the Alcoa4 support consisted of smaller particles compared to the Alcoa5 and Alcoa6 supports.

Table 4.5 The dimensions and linear shrinkage of Alcoa4, Alcoa5 and Alcoa6 supports

\begin{tabular}{ccc}
\hline Support & Outer diameter $(\mathbf{m m})$ & Linear Shrinkage (\%) \\
\hline Alcoa4 & 20.58 & 3.02 \\
Alcoa5 & 20.70 & 2.99 \\
Alcoa6 & 20.74 & 2.78 \\
\hline
\end{tabular}

Steenkamp ${ }^{13}$ stated that: "Smaller particles packed equivalently will produce an equivalent shrinkage in a shorter time because of more contact points per unit volume, and a larger driving force". Thus, the results in Table 4.5 confirm that the supports consisted of different particle sizes due to the difference in the linear shrinkage. 


\subsection{Mercury porosimetry}

The porosity and the median pore diameter differed for the fractions used according to Table 4.6. The fractions higher up in the column (lower numbered fractions) resulted in a support structure with a lower porosity and a smaller pore diameter. The same observation was made for the Alcoa1 - Alcoa3 supports (Table 4.4). The Alcoa1 and the Alcoa4 supports consisted of smaller particles overall while the Alcoa3 and Alcoa6 consisted of larger particle sizes. According to Herring's scaling law, fine particles promote faster sintering which explains the more rapid structural shrinkage obtained from the dimensions of the Alcoa4 support structure (Table 4.6) when compared to the A/coa5 and A/coa6 supports. ${ }^{14}$

Table 4.6 The porosity, median pore diameter and water permeability of the A/coa4-Alcoa6 supports. The supports were manufactured with powder pretreated with the acid and column treatment. The Alcoa4 permeability data is not available due to the fact that the membrane broke.

\begin{tabular}{cccc}
\hline Support & $\begin{array}{c}\text { Median pore diameter } \\
(\mathrm{nm})\end{array}$ & $\begin{array}{c}\text { Porosity } \\
(\%)\end{array}$ & $\begin{array}{c}\text { Water permeability } \\
\left(\mathrm{L} \mathrm{m}^{2} \mathrm{~h}^{-1} \mathrm{bar}^{-1}\right)\end{array}$ \\
\hline Alcoa4 & 117.5 & 37.6 & $(\mathrm{a})$ \\
Alcoa5 & 120.1 & 39.2 & 27.75 \\
Alcoa6 & 122.0 & 40.5 & 28.22 \\
\hline
\end{tabular}

(a) - Membrane broke

The characteristics of the support structure was influenced by the removal of fine particles (before the column fractionation) as can be seen when Table 4.4 and Table 4.6 are compared. The removal of the fine particles (Alcoa4 - Alcoa6) led to an increase in porosity for fractions 6 and 7 as well as for fractions 8 and 9 (Alcoa2 vs. Alcoa5, Alcoa3 vs. Alcoa6). The removal of the fine particles resulted in the elimination of the dense inner layer created by the deposition of the fine particles on the inside of the tubular structure during centrifugal casting, resulting in an increased porosity. The porosity decrease for Alcoa4 (compared to Alcoa1) was not anticipated as a porosity increase would be expected when finer particles are removed. The elimination of the 
dense inner layer created by the fine particles (in effect smaller pores) resulted in an increased median pore diameter for all the fractions.

The pore size distributions of the Alcoa4 - Alcoa6 fractions were monomodal as illustrated in Figure 4.12. The Alcoa4 fraction had the narrowest distributions and the smallest median pore size while the A/coa6 fraction had the widest distributions. These results are similar to the A/coa1 - Alcoa3 fractions (Figure 4.10) confirming that the fractions situated at the top of the column during the column treatment (lower numbered fractions) consisted of a narrower particle size distribution than the fractions at the bottom (higher numbered fractions). This has been attributed to the gravitational settling occurring as well as agglomeration as described in Section 4.3.4.2.1.

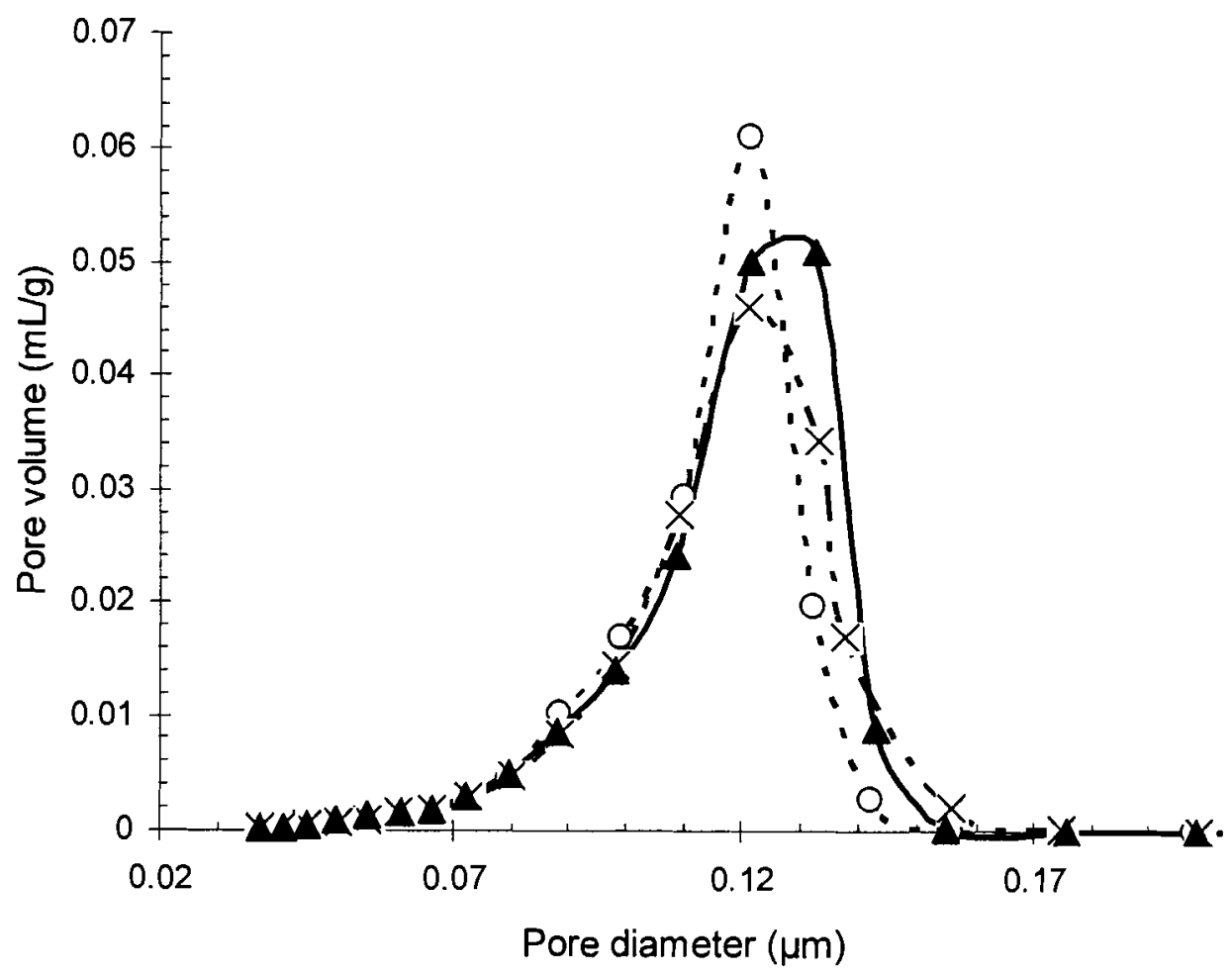

Figure 4.12 Pore volume as a function of pore diameter for the Alkoa4, Alcoa5 and Alcoa6 supports sintered at $1150^{\circ} \mathrm{C}$.

$$
\begin{array}{cc}
-0-- & : \text { Alkoa4 } \\
-x-- & : \text { Alcoa5 } \\
-\Delta- & : \text { Alcoa6 }
\end{array}
$$




\subsection{Water permeability}

The pore size and the porosity influenced the water permeability as seen in Table 4.6, which is in agreement with the Hagen-Poiseule equation. ${ }^{13}$ The larger pore size and porosity of the Alcoa6 compared to the Alcoa4 and Alcoa5 supports resulted in increased water permeability.

From the larger pore size and porosity of the Alcoa6 and Alcoa5 compared to the Alcoa3 and Alcoa2 supports, a higher permeability would be expected for the A/coa6 and Alcoa5 supports. The opposite was, however, observed. This could be due to the defective inside surface of the Alcoa1- Alcoa3 membrane structures. Although the inner surface of these membranes was denser, the defective surface structure could result in an increased permeability. The effect of these large amounts of fine particles during sintering can not be excluded. During sintering, the differences in sintering rates present between the fine particles situated at the inside wall and the outside structure of the support can result in defects in the support structure due to the shrinkage rate difference. ${ }^{3}$ The defects present in the support structure of the supports with large amounts of fine particles (Alcoa1, Alcoa2, Alcoa3) would ultimately result in an increased permeability as observed when the permeability values of Table 4.4 (Alcoa1, Alcoa2, Alcoa3) and Table 4.6 (Alcoa5, Alcoa6) are compared.

\subsubsection{SEM images and photographs}

SEM images of the inner surface of the A/coa4, Alcoa5 and A/coa6 supports sintered at $1150^{\circ} \mathrm{C}$ at a high magnification are shown in Figure 4.13 (a) - (c) and a low magnification of the Alcoa4 surface is shown in Figure 4.13 (d).

On the high magnification image of the Alcoa6 (c) surface, slightly larger particles were visible than on the surface of the Alcoa4 (a) and Alcoa5 (b) supports which appeared to be similar in size, confirming the mercury intrusion and water permeability results. The Alcoa6 support had a homogeneous particle packing, while "pin holes" and "cracks" were visible on the Alcoa5 (b) surface and even more so on the Alcoa4 (a) surface. This observation indicates that a larger amount of fine particles were present on the inside layer of the A/coa4 support after centrifugation, which resulted in defect formation during sintering of the compact due to the differences in the sintering rate of the fine particles on the inside and large particles on the outside of the tubular structure. $^{5}$ 
When the Alcoa4, Alcoa5 and Alcoa6 support surfaces are compared to the surfaces of the supports prepared from Alcoa powder pretreated with only the acid treatment (Figure $4.9 a$ of Section 4.3.3.1), it is clear that the combined acid and column treatments (A/coa4, A/coa5 and Alcoa6) reduced the large variation of particle sizes which were visible on the surface of the support in Figure 4.9a. This illustrates the fact that some degree of fractionation occurred during the column treatment. A similar observation is made when the low magnifications of the Alcoa4 surface (Figure 4.13d) and the support prepared with only acid treatment of the Alcoa powder (Figure $4.9 b$ ) are compared. Severe surface cracking was noticeable for the Alcoa support produced without the column fractionation (Figure 4.9b), while the integrity of the Alcoa4 surface (acid and column treatment) was much better. This illustrates once again that some fractionation occurred during the column treatment, which resulted in a support with less defects due to a narrower PSD. ${ }^{3}$ 


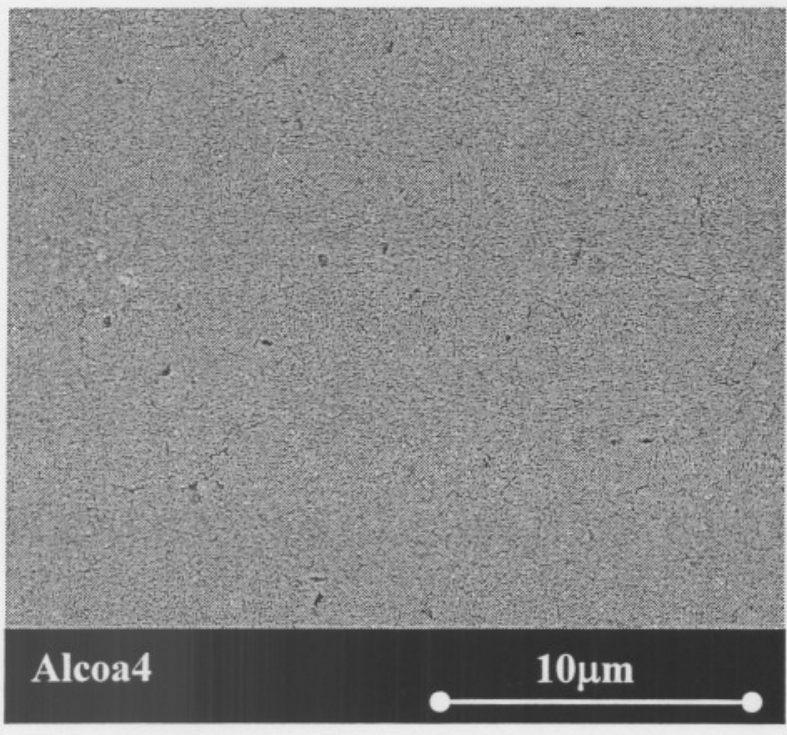

(a)

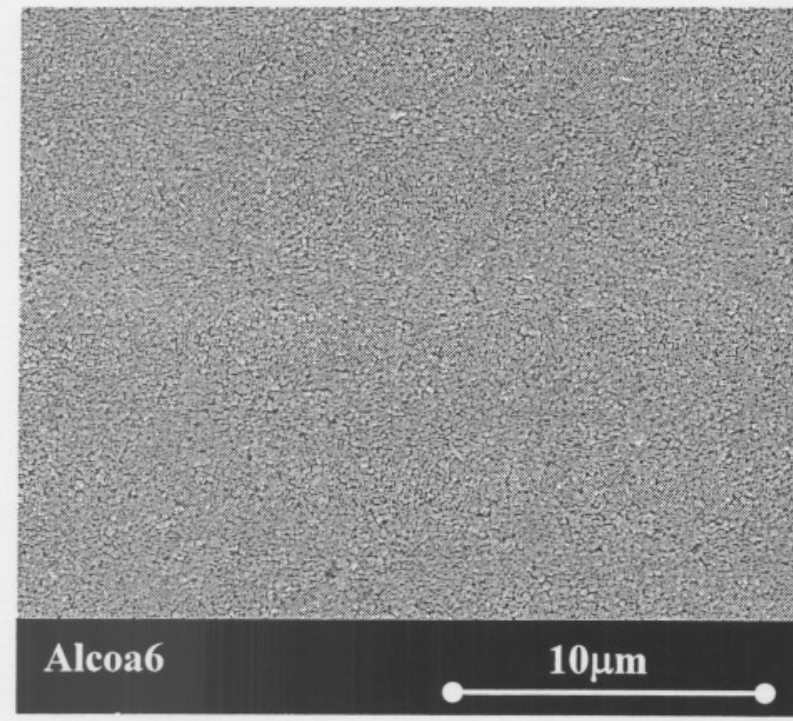

(c)

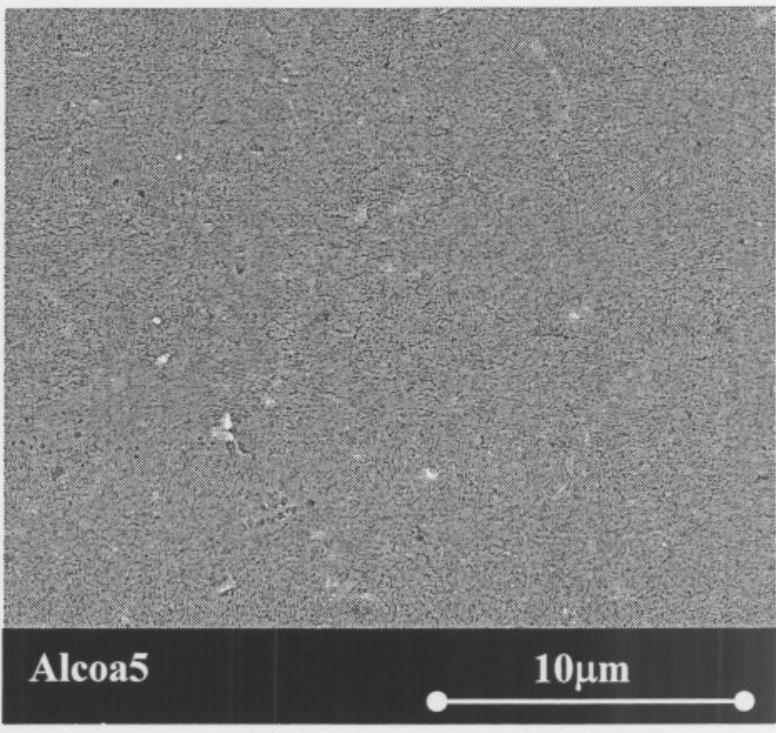

(b)

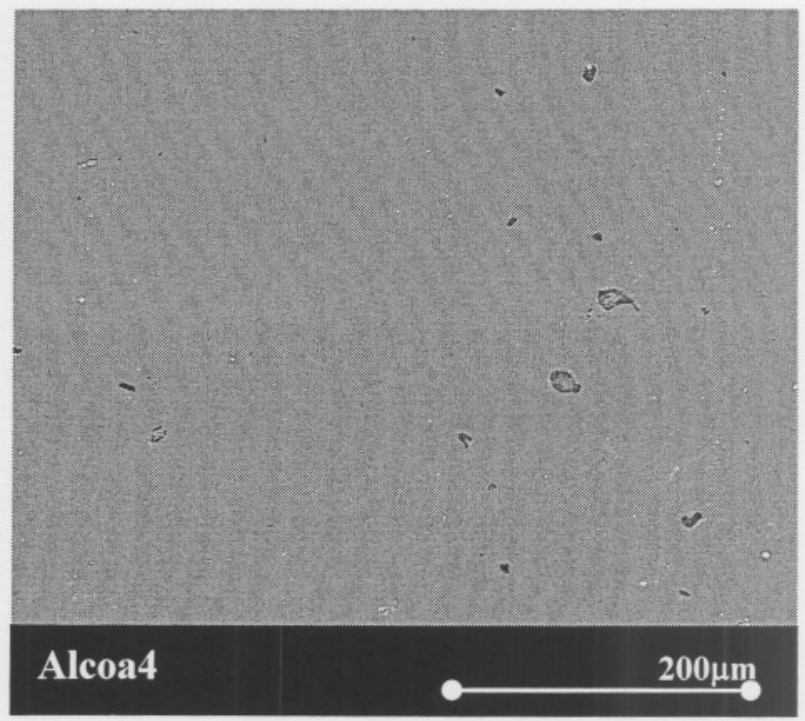

(d)

Figure 4.13 SEM images of (a) Alcoa4, (b) Alcoa5 and (c) Alcoa6 supports sintered at $1150^{\circ} \mathrm{C}$ and (d) a lower magnification of the Alcoa4 support

Figure 4.14a shows a photograph of the Alcoa5 and Alcoa6 (Alcoa6 at the bottom). The fractions used for the manufacture of the Alcoa5 and Alcoa6 resulted in supports with smooth inner surfaces and defect-free outer structures. When this image is compared to a support where only the column treatment was performed without the acid treatment (Figure 4.11 of Section 4.3.4.2.3), it is clear that the inner surface of the support has improved due to the removal of the fine particles with the acid treatment. 
When attempting to repeat the method used to prepare the defect-free Alcoa6 membrane, the same internal cracking observed in Figure 4.11 was obtained (Figure 4.14b). Although the manufacturing process for Figure 4.14a and Figure 4.14b were identical, the support structures differed, indicating the non-repeatability of the acid treatment and column fractionation employed in preparation of the starting powder.

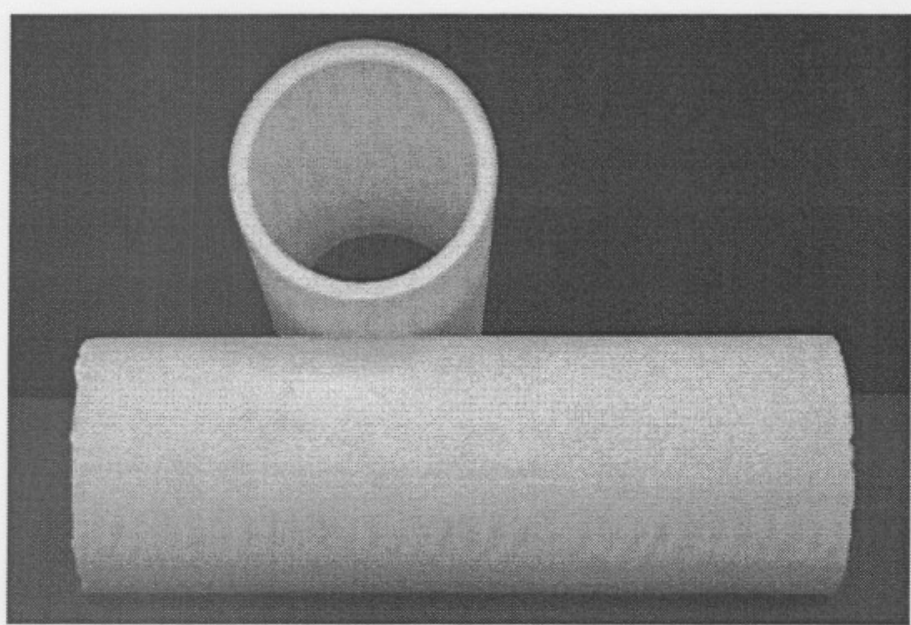

(a)

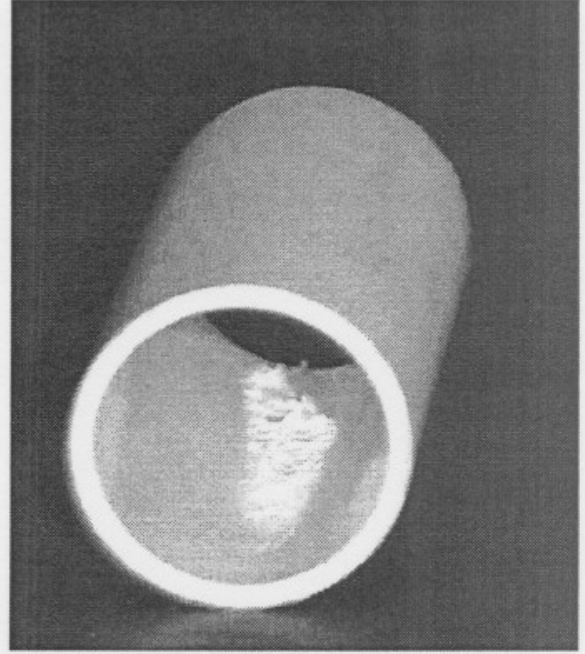

(b)

Figure 4.14 Photographs of (a) Alcoa5 and Alcoa6 (Alcoa6 at the bottom), (b) a support manufactured in an identical manner to the Alcoa5 and Alcoa6 supports.

\subsection{Conclusions}

From the study investigating the possibility of replacing the AKP powder with the less expensive Alcoa CT 3000 SG $\alpha$-alumina powder the following conclusions can be made:

- The particle size distribution (PSD) of the Alcoa powder is too large for the manufacture of tubular centrifugal casted ceramic membrane supports.

- The amounts of particles removed by means of the $\mathrm{HCl}$ and $\mathrm{HNO}_{3}$ acid treatments differed slightly. No particles were suspended at a pH above 2 after 48 hours for both $\mathrm{HCl}$ and $\mathrm{HNO}_{3}$ treatments, while below $\mathrm{pH} 2$ more than $10 \%$ of the powder was still in suspension. For the $\mathrm{HCl}$ treatment below $\mathrm{pH}$ 2, the amount of particles suspended was not influenced by the $\mathrm{pH}$ but rather by the solid loading (largest amount at $200 \mathrm{~g}$ solid loading). The largest 
amount of particles was in suspension at $\mathrm{pH} 1.5$ for the $\mathrm{HNO}_{3}$ treatment after 48 hours while the solid loading had little influence on the amount of particles in suspension. According to the particle size analysis and SEM analyses, a large amount of small particles were present in the suspensions while the larger particles settled.

- The polymer concentration influenced the Malvern particle size analysis. A low polymer concentration resulted in agglomeration.

- The column treatment resulted in some degree of fractionation, but the Malvern Mastersizer instrument was unable to measure the slight differences present in these fractions. The repeatability of the fractionation method was low. The degree of fractionation was confirmed by the defect-free supports produced using combined fractions. According to the results, the fractions situated at the top of the column consisted of smaller particles than the fractions at the bottom.

- The removal of fine particles from the untreated Alcoa powder is crucial in obtaining a defect free inner surface. The broad and bimodal PSD of the Alcoa CT 3000 SG powder is the major reason for cracking and warping of the support due to differences in sintering rates present between particles in different regions of the powder compact during sintering. Further studies are required to ensure repeatable fractionation of the Alcoa powder, which is essential when manufacturing defect free membrane supports. 


\subsection{References}

1 P.M.Biesheuvel, Ph.D. Thesis, Universiteit Twente, The Nederlands, 1999, Porous ceramic membranes suspension processing, mechanical and transport properties, and application in the osmotic tensiometer, Summary

${ }^{2}$ G.Tari, J.M.F.Ferreira, A.T.Fonseca, O.Lyckfeldt, Influence of particle size distribution on colloidal processing of alumina, Journal of the European Ceramic Society, 18, 1998, 249

3 J.Ma, LC.Lim, Effect of particle size distribution on sintering of agglomerate-free submicron alumina powder compacts, Journal of the European Ceramic Society, 22, 2002, 2197

${ }^{4}$ N.Das, H.S.Maiti, Effect of size distribution of the starting powder on the pore size and its distribution of tape cast alumina microporous membranes, Journal of the European Ceramic Society, 19, 1999, 341

${ }^{5}$ Ken Darcovich, Laurent Béra, Kazunari Shinagawa, Particle size distribution effects in an FEM model of sintering porous ceramics, Materials Science and Engineering, A341, 2003, 247

${ }^{6}$ P.Mikkola, P.Ylha, E.Lavanen, J.B.Rosenholm, Effect of impurities on the dispersion of alphaalumina powder, Ceramics International, 30, 2004, 291

7 A.R.Studart, V.C.Pandofelli, E.Tervoort, L.J.Gauckler, Direct coagulation casting of alumina suspensions using Jack Bean as a urease source, Journal of the American Ceramic Society, 85(7), 2002, 1695

${ }^{8} \mathrm{http}: / /$ www.alumina.alcoa.com/alumina/pdf/cal_refr.pdf.

9 J.Hofman-Zuter, Chemical and thermal stability of (Modified) Mesoporous ceramic membranes, Ph.D Thesis, Universiteit Twente, Chapter 2, Theoretical Background, 1995, pp17 
${ }^{10}$ Y.Koreeda, Y.Hirata, S.Sameshima, Analysis of particle connection in a two-component powder compact by electric conductivity measurements, Journal of Ceramic Processing, 5(4), 2004, 337342

11 B.P.Singh, S.Bhattacharjee, L.Besra, D.K.Sengupta, Evaluation of dispersibility of aqueous alumina suspension in presence of Darvan C, Ceramics International, 30, 2004, 939

${ }^{12}$ W.D.Kinery, H.K.Bowen, D.R.UhImann, Introduction to Ceramics, Second Edition, Chapter 10, Grain growth, sintering and verification, Wiley Interscience, 1976, pp. 469

${ }^{13}$ G.C.Steenkamp, Centrifugal casting and coating of tubular ceramic membrane supports, M.Sc. Thesis, Potchefstroom University for Christian Higher Education, South Africa, 2000, Chapter 2: Centrifugal casting, pp. 25

14 Y.Chiang, D.Birnie, W.D.Kingery, Physical ceramics: Principles for ceramic Science and engineering, Wiley, 1997, Chapter 5, Single-Phase sintering, 403 


\section{Chapter 5}

\section{Evaluation}

and

\section{Recommendations}

\subsection{General discussion}

Centrifugal casted tubular membrane supports were manufactured using three different Sumitomo AKP $\alpha$-alumina particle sizes and a range of sintering temperatures to study the effect of particle size and sintering temperature on the properties of the tubular support. All membranes had a smooth inside surface and a homogeneous particle packing.

The optimization of the tubular supports was achieved when compared to previous studies. In one of our own previous studies by Steenkamp. ${ }^{2}$, we obtained a permeability of $28 \mathrm{~L} . h .^{-1}{ }^{-1} \mathrm{bar} .{ }^{-1} \mathrm{~m} .{ }^{-2}$, a porosity of $36 \%$ and a pore radius of $99 \mathrm{~nm}$ for an AKP-15 support sintered at $1200^{\circ} \mathrm{C}$. In this study, the permeability was improved to $41 \mathrm{~L} . h .^{-1} \mathrm{bar}^{-1} \mathrm{~m}^{-2}$, while the porosity increased to $37 \%$ and the pore diameter to $167 \mathrm{~nm}$. The same improvements were obtained for supports sintered at different temperatures as well as the AKP-30 supports in the study by Steenkamp, ${ }^{2}$ compared to the AKP- 
30 supports in this study. The permeance of the tubular supports in this study also compares favorably when evaluated alongside similar studies in literature. ${ }^{3}$

In Figure 5.1, the effects that the particle sizes $(0.25,0.31$ and $0.61 \mu \mathrm{m})$ and sintering temperatures had on the pore size, porosity, water permeability, linear shrinkage (dimensions) and mechanical strength of the support structures are summarized.

There was an increase in pore size, porosity and permeability, while there was a decrease in mechanical strength and linear shrinkage with increasing particle size of the AKP powder for supports sintered at the same temperatures. The centrifugal casting of the AKP-50 $(0.25 \mu \mathrm{m}$ particles) supports resulted in a different particle packing than the AKP-30 $(0.31 \mu \mathrm{m}$ particles) and

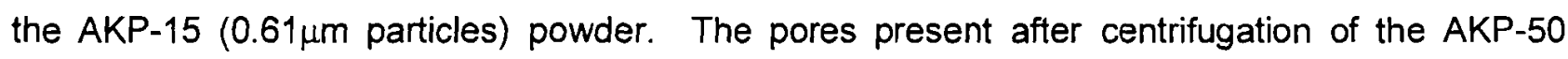
supports were surrounded by 6 particles (grains), while in the case of the AKP-30 and AKP-15 supports the pores were surrounded by less than six particles. ${ }^{1}$ There was an increase in linear shrinkage and mechanical strength for all the supports, but a decrease in porosity and permeability with increasing sintering temperature. The pore size of the AKP-50 supports, however, remained constant, irrespective of the sintering temperature, while the pore size of the AKP-30 and AKP-15 supports decreased with increasing sintering temperature. These observations were in agreement with studies performed by numerous authors. ${ }^{2,3,4}$

According to Biesheuvel et al., ${ }^{5}$ the AKP-15 powder had the widest particle size distribution (PSD) while the AKP-50 had the narrowest. This was confirmed in this study where the supports manufactured from the AKP-15 powder had the widest pore size distribution, while the supports made from the AKP-50 powder had the narrowest distribution, considering the direct proportionality between PSD and pore size distribution. A similar pore size distribution was shown by Das et al. ${ }^{6}$ For all the supports manufactured, there was a narrowing of the pore size distribution with increasing sintering temperature. 


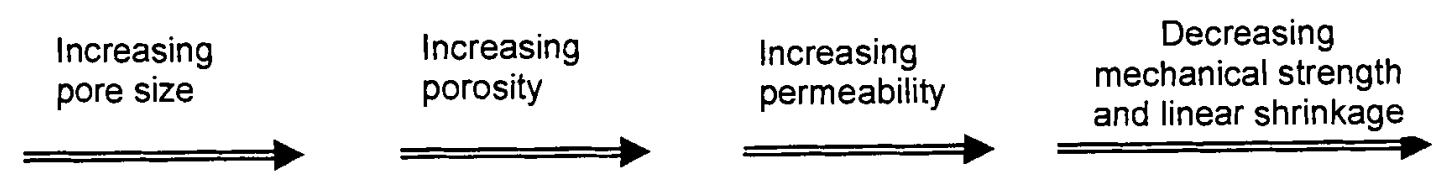

Sintering of centrifugal casted powder compact at a similar temperature

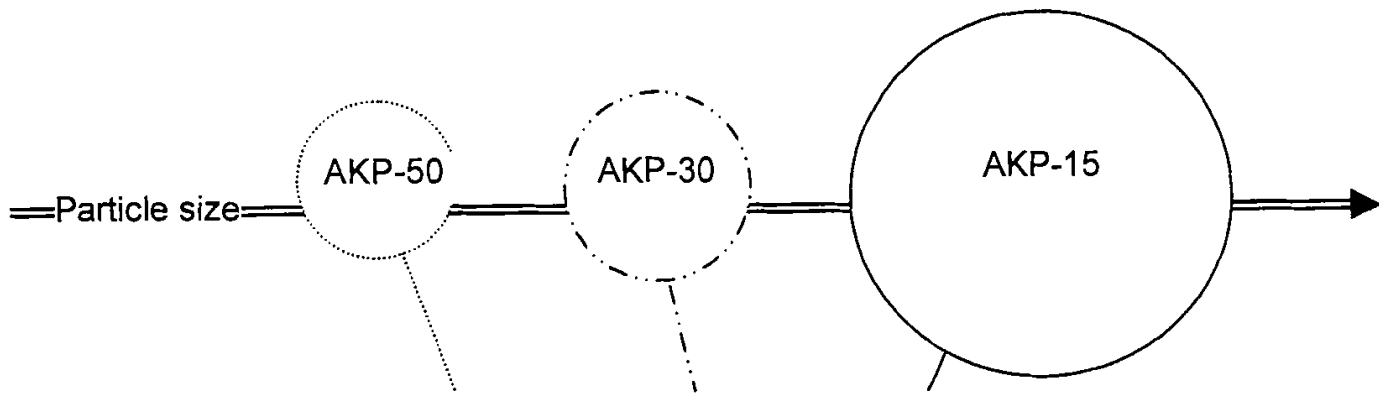

Centrifugal casting at $17000 \mathrm{rpm}$

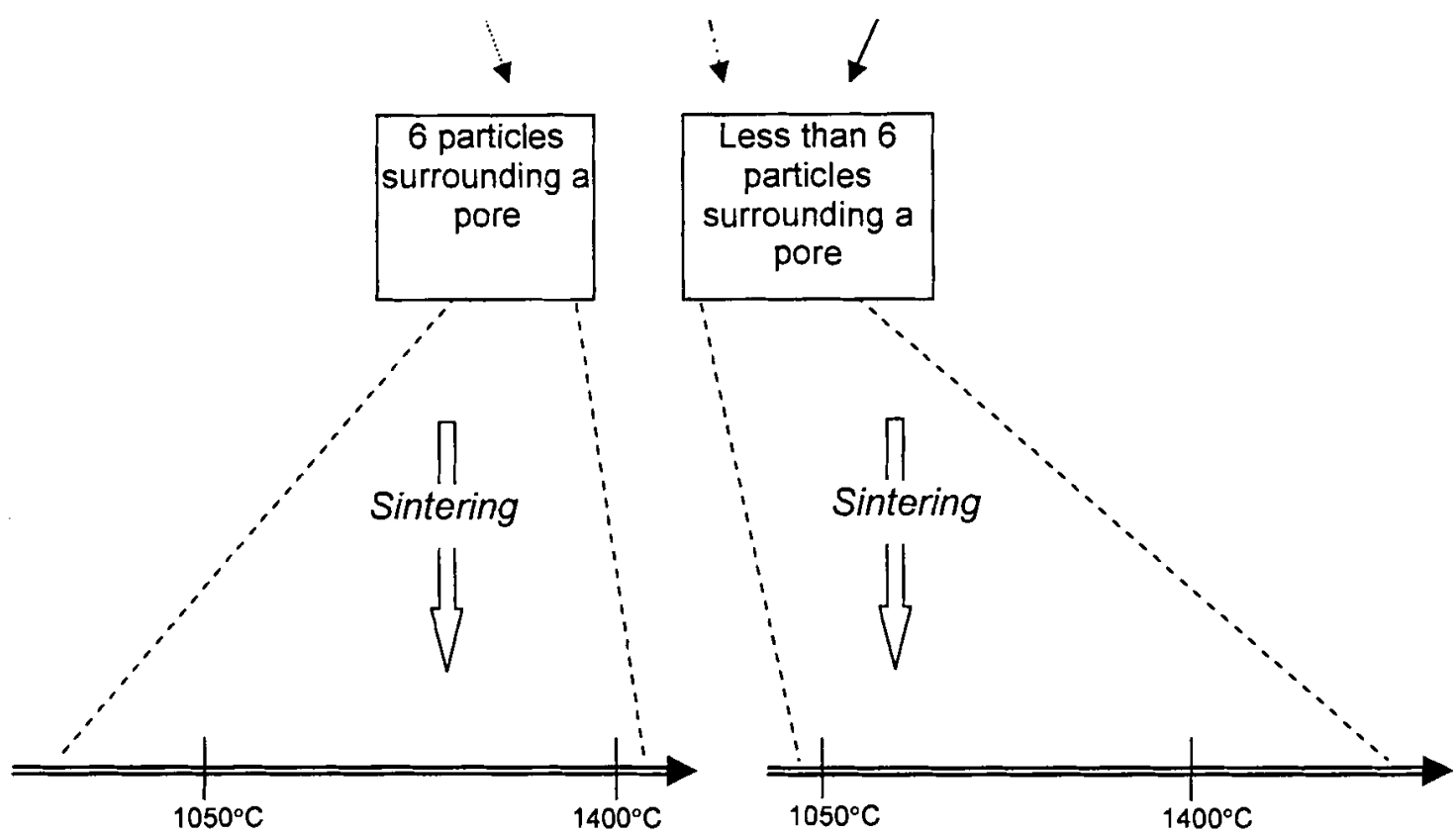

With temperature increase

With temperature increase

- Constant pore size

- Decreasing pore size

- Decreasing porosity

- Decreasing permeability

- Increasing linear shrinkage

- Increasing mechanical strength

- Decreasing porosity

- Decreasing permeability

- Increasing linear shrinkage

- Increasing mechanical strength

Figure 5.1 The effects of particle size and sintering temperature on the support properties. 
When the concentration of polymer (APMA) which is used to stabilize the colloidal suspension (used to produce the supports) was increased, it resulted in the evolution of "cracks", which consequently led to an increase in pore sizes, porosity and permeability, while a decrease in structural strength was observed. These results, obtained with the AKP-30 $(0.31 \mu \mathrm{m})$ support, were also confirmed by literature. ${ }^{7}$ In this study the variation in the sintering rate did not noticeably influence the support properties (permeability, pore diameter, porosity and strength).

A study concerning the possible replacement of the expensive Sumitomo AKP-powder range with the less expensive Alcoa CT 3000 SG $\alpha$-alumina powder was undertaken. Manufactured supports using untreated Alcoa powder resulted in structural cracking and warping during sintering of the centrifugal casted powder compact due to the bimodal, and hence wide, particle size distribution of the Alcoa powder. ${ }^{8}$ The large amount of fine particles present in the Alcoa powder resulted in a defective inner surface and was the main reason for the structural cracking during sintering. The removal of a large amount of fine particles was attained by suspending the powder in an acid medium. Separation of the fine and heavy particles was obtained by the differential gravitational settling. Further fractionation was achieved by suspending the heavy fraction, obtained from the acid treatment, in a fractionating column. The size range of the fractions obtained by the column treatment could not be detected by means of particle size analysis, but three supports manufactured from three different combinations of fractions suggested that some degree of fractionation occurred during the column fractionation. Fractions situated in the middle regions of the column were combined to manufacture the defect-free support. Fractions higher-up in the column would consist of too many fine particles, while the PSD of the fractions closer to the bottom of the column would be too large and defects would occur during sintering of the powder compacts. However, numerous attempts to repeat these results were unsuccessful, suggesting that the fractionation by means of the acid and column treatments were unpredictable with a low repeatability. Repeatable fractionation of the untreated Alcoa powder is essential if the cheaper Alcoa powder is to replace the more expensive AKP powder range for the manufacturing of centrifugal casted membrane supports.

\subsection{Conclusion}

The effects of particle size, sintering temperature, polymer concentration and sintering rate on the properties of centrifugal casted ceramic membrane supports made from Sumitomo AKP powder were optimized. The AKP-15 (largest particle size) supports sintered at $1050^{\circ} \mathrm{C}$ resulted in the 
largest permeability, pore size and porosity, while the mechanical strength of these support were the lowest. The smallest powder size (AKP-50) sintered at the highest sintering temperature resulted in the support with the lowest permeability and porosity, smallest pore size and the largest mechanical strength. The higher polymer concentration led to an increased permeability, porosity and pore diameter, but a decreased mechanical strength.

The possibility of replacing the expensive Sumitomo AKP powder range with the Alcoa CT 3000 SG powder depends on the repeatable fractionation of the Alcoa powder. While some improvement was obtained by reducing the PSD of the Alcoa powder, the repeatability of the process remains a major hurdle.

\subsection{Recommendations}

According to this study, the following aspects require further elucidation:

- Optimization of the centrifugal casted supports by investigating the influence of sintering time and an in-depth study of the effect of sintering rate on the properties of the supports.

- Addition of carbon particles and fibers to increase the porosity of the ceramic supports

- Investigation of possible methods to determine the surface roughness and chemical properties of the support surface which will assist in understanding and improving the zeolite attachment on the inside surface of the tubular support.

- In Chapter 3 it was shown that a powder with a large particle size distribution resulted in a support structure with a large pore size distribution, while a narrow particle size distribution results in a support with a narrower pore size distribution. By means of this observation it would be interesting to manufacture supports consisting of various particle sizes and distributions which are sintered at similar temperatures and then determine their pore size distributions in order to determine whether there is a relationship and what the relationship might be between the particle size distribution and pore size distribution. If such a relationship is observed, the method could be employed to determine the particle size distributions of powders that are unknown.

- The development of a continuous fractionation technique in order to make the Alcoa CT 3000 SG powder suitable for centrifugal casted membrane manufacturing.

- The manufacturing of long membranes with a small diameter in order to maximize the surface area per volume and thus increasing the permeability performance. 


\subsection{References}

I Y.Chiang, D.Birnie, W.Kingery, Physical ceramics: Principles for ceramic Science and engineering, Wiley, 1997, Chapter 5, Single-Phase sintering, 409

${ }^{2}$ G.C.Steenkamp, Centrifugal casting and coating of tubular ceramic membrane supports, M.Sc. Thesis, Potchefstroom University for Christian Higher Education, South Africa, 2000, Chapter 2: Centrifugal casting, pp. 25

${ }^{3}$ K.Kim, S.Cho, K.Yoon, J.Kim, J.Ha, D.Chun, Centrifugal casting of alumina tube for membrane application, Journal of Membrane Science, 199, 2002, 69

${ }^{4}$ C.H.Chen, K.Takita, S.Honda, H.Awaji, Fracture behouvior of cylindrical alumina with pore gradient, Journal of the European Ceramic Society, 25, 2005, 385

${ }^{5}$ P.M.Biesheuvel, H.Verweij, Microdivers to study sedimentation in polydisperse concentrated colloidal suspensions, A/ChE Journal, 47(9), 2001, 1969

${ }^{6}$ N.Das, H.S.Maiti, Effect of size distribution of the starting powder on the pore size and its distribution of tape cast alumina microporous membranes, Journal of the European Ceramic Society, 19, 1999, 341

7 J.M.Benito, A.Conesa, F.Rubio, M.A.Rodriguez, Preparation and characterazation of tubular ceramic membranes for treatment of oil emulsions, Journal of the European Ceramic Society, 25, 2004, 1895

${ }^{8} \mathrm{J.Ma}$, LC.Lim, Effect of particle size distribution on sintering of agglomerate-free submicron alumina powder compacts, Journal of the European Ceramic Society, 22, 2002, 2197 